\title{
Representações Irredutíveis De Grau Dois Da Primeira Álgebra De Weyl
}

\author{
César Augusto Rodríguez Duque
}

\author{
DissertaÇÃO/TESE APRESENTADA \\ $\mathrm{AO}$ \\ Instituto DE MATEMÁtica E EstatísticA \\ $\mathrm{DA}$ \\ Universidade de SÃo PAUlo \\ PARA \\ OBTENÇÃO DO TÍTULO \\ DE \\ Doutor EM CIÊNCIAS
}

Programa: Matemática

Orientador: Prof. Dr. Alexandre Grishkov

Durante o desenvolvimento deste trabalho o autor recebeu auxílio financeiro da CAPES

São Paulo, novembro de 2015 


\section{Representações Irredutíveis De Grau Dois Da Primeira Álgebra De Weyl}

Esta é a versão original da dissertação/tese elaborada pelo candidato, César Augusto Rodríguez Duque, tal como submetida à Comissão Julgadora. 


\section{Representações Irredutíveis De Grau Dois Da Primeira Álgebra De Weyl}

Instituto de Matemática e Estatística da Universidade de São Paulo.

Comissão Julgadora:

- Prof. Dr. Alexandre Grishkov (orientador)- IME-USP

- Prof. Dr. Alexandre Ananin - ICMC

- Prof. Dr. Kostiantyn Iusenko - IME-USP

- Prof. Dr. Artem Lopatin - UNICAMP

- Prof. Dr. Alexandr Kornev - UFABC 


\section{Agradecimentos}

Ao professor Doutor Alexandre Grishkov pela sua orientação, compreensão e acompanhamento no desenvolvimento do doutorado. A minha esposa Sandra pelo apoio e a minha família. 


\section{Resumo}

Duque César A. R. Representações irredutíveis de grau dois da primeira álgebra de Weyl. Tese Doutorado - Instituto de Matemática e Estatística, Universidade de São Paulo, São Paulo, novembro 27 de 2015.

Sejam $K$ um corpo comutativo de caraterística zero. Definimos a álgebras associativa sobre $K$ com dois geradores $p, q$ onde $p q-q p=1$, como a primeira álgebra de Weyl, denotaremos esta por $A_{1}$. As representações irredutíveis de grau um de dimensão infinita de $A_{1}$, foram descritos por R. Block em (Block, 1981). Baseados nesta ideia, são descritas as representações irredutíveis de grau dois de dimensão infinita de $A_{1}$. No capítulo 1 são estudadas a representações da localização $S^{-1} A_{1}=\mathfrak{B}$ onde $S=K[q]-\{0\}$, ver (Block , 1981). Também apresentamos algumas definições e resultados relevantes para $A_{1}$, os quais estabelecem uma relação entre as representações de álgebras de Lie nilpotente e as representações da enésima álgebra de Weyl $A_{n}$, ver (Dixmier , 1959). No segundo capítulo é abordado o estudo da estrutura para $A_{1}$-módulos de grau dois de dimensão infinita, obtendo uma descrição completa destes módulos. Usando esta estrutura é dada uma relação entre uma classe de $S l_{2}$-módulos de dimensão infinita e os $A_{1}$-módulos de grau dois. Finalmente, no capítulo 3 são dados alguns fatos importares sobre a estrutura do $\operatorname{Ext}^{1}(M, N)$, onde $M$ e $N$ são $A_{1}$-módulos irredutíveis de dimensão infinita com graus $n_{1}$ e $n_{2}$ repectivemente.

Palavras-chave: álgebras de Weyl, módulos irredutíveis de grau n, Ext $t^{1}$, localização de anéis, polinômios irredutíveis, domínios de ideais principais. 


\section{Abstract}

Duque César A. R. Irreducible Representations the two deg of the first Weyl algebra. Tese Doutorado - Instituto de Matemática e Estatística, Universidade de São Paulo, São Paulo, 27 de novembro de 2015.

Let $K$ be a commutative field such of zero characteristic. The associtive algebras from $K$ whit two geradors $p, q$ shuch that $p q-q p=1$ is the first Weyl algebra and it algebra going to denoted for $A_{1}$. The structure of irreducible representations of degree one of infinite dimensional of $A_{1}$, studied by R.Block (Block , 1981) on 1981. Based in this paper, we characterize the structure of degree two of irreducible representations of infinite dimensional of $A_{1}$.

In the first chapter, we speak of localization rings and defined $\mathfrak{B}$, we also give tools and definitions needed over Weyl algebras and nilpotent Lie algebras. In the second chapter we give the review for to the problem of $A_{1}$-modules of degree two of infinite dimensional. At the end of the thesis we calculate the $\operatorname{Ext}^{1}(M, N)$, by $M$ e $N$ irreducibles $A_{1}$-modules of degree $n$.

Keywords: Weyl algebras, irreducibles modules of the degree $n, E x t^{1}$, localization rings and principal ideal domain. 


\section{Introdução}

As álgebras de Lie foram introduzidas por Sophus Lie e um dos grandes problemas destas estruturas é a classificação destas álgebras, assim como o estudo das representações das mesmas. Ao longo da história, o estudo das classificações das álgebras de Lie divide-se em três tipos, as álgebras de Lie semisimples, as solúveis e as que não são semisimples e não são solúveis. O teorema de Levi-Malcev que foi demonstrado em 1945 (Maltsev , 1962), (Levi , 1905), nós diz que, toda álgebra de Lie de dimensão finita pode-se escrever como a suma direta entre de uma subálgebra semisimples e seu radical solúvel. Portanto, no estudo das álgebra de Lie de dimensão finita sobre um corpo de caraterística zero os dois grupos; as álgebras de Lie semisimples e as solúveis, são de grande importância. As primeiras foram classificadas pelo teorema de Cartan: toda álgebra de Lie semisimples de dimensão finita sobre um corpo de caraterística zero, pode-se escrever como soma de ideais simples, assim o problema de classificação de álgebras de Lie semisimples se reduz a classificação das álgebras de Lie simples, as quais foram classificadas na década dos 90 por Killin, Cartan e outros grupos de matemáticos da época (Cartan , 1894). Nas segundas, estão contidas as álgebras de Lie nilpotente.

Neste sentido, temos a álgebra de Lie nilpotente de dimensão três $\mathfrak{h}$, gerada por $x, y, z$, chamada álgebra de Heisenberg, com produto de Lie dada por $[x, z]=[y, z]=0$ e $[x, y]=z$. Em 1963, J. Dixmier nos trabalhos (Dixmier , 1963), (Dixmier , 1959), introduz os ideais primitivos das álgebras de Lie nilpotentes, estes ideais estão definidos como o núcleo de uma representação irredutível da álgebra envelopante. No caso da álgebra de Heisenberg, os ideais primitivos são da forma $I_{\lambda}=U \mathfrak{h}(z-\lambda)$ com quociente da forma $U \mathfrak{h} / U \mathfrak{h}(z-\lambda)$, que é gerado por dois elementos $\bar{x}, \bar{y}$ tais que $[\bar{x}, \bar{y}]=\lambda$. Para $\lambda \neq 0$ gera-se á primeira álgebra de Weyl $A_{1}$, que tem este nome devido ao matemático Hermann Weyl. A álgebra $A_{1}$ é gerada por dois elementos $p$ e $q$ tais que $[p, q]=1$, assim é possível estabelecer um isomorfismo entre $A_{1}$ e os quocientes primitivos da álgebra envelopante da álgebra de Heisenberg $U \mathfrak{h} / I_{\lambda}$.

Em (Dixmier , 1963), J. Dixmier mostrou que existe uma correspondência entre o quociente da álgebra envelopante $U g$, de uma álgebra de Lie nilpotente de dimensão finita $g$, por um ideal primitivo, chamado de quociente primitivo, e $A_{n}$, para algum $n$. A álgebra $A_{n}$, é a enésima álgebra de Weyl com $2 n$ geradores; $x_{i}, \cdots, x_{n}, y_{i}, \cdots, y_{n}$ onde $\left[x_{i}, y_{j}\right]=\delta_{i j}$, 
$\left[x_{i}, x_{j}\right]=0$ e $\left[y_{i}, y_{j}\right]=0$. Isto faz que o estudo das representações da álgebra de Weyl seja importante, dentro da teoria das representações das álgebras de Lie nilpotentes.

Anos depois , em 1968 o mesmo matemático J. Dixmier (Dixmier , 1968), estuda a profundidade a estrutura e as propriedades da primeira álgebra de Weyl $A_{1}$ sobre um corpo de caraterística zero. No final deste trabalho Dixmier deixou uma lista de 6 problemas em aberto. Em 1975, Joseph and Stein dão solução aos problemas 3 e 6, usando ferramentas de McConnell and Robson (McConnell e Robson , 1987), ver (Joseph , 1975), em 2005, V. V. Bavula da solução ao problema 5, ver (Bavula , 2005). Restam ainda três problemas em aberto, a saber os problemas 1, 2 e 4 . O problema 1 conhece-se como a conjetura de Dixmier: O conjunto de $\operatorname{End}\left(A_{1}\right)$ coincide com o conjunto de $A u t\left(A_{1}\right)$.

A classificação das representações da primeira álgebra de Weyl $A_{1}$ foi dada por R. BLock no ano $1981 \mathrm{em}$ (Block , 1981), usando a localização de $A_{1}$ pelo conjunto $S=K[q]-\{0\}$, e as propriedades do domínio de ideais principais $\mathfrak{B}=S^{-1} A_{1}$, ver (Amitsur , 1954), (Ore , 1933). Da localização anterior obtemos $\mathfrak{B}=K(q)[p]$ onde o comutador $[f(q), p]=f^{\prime}(q)$, para $f \in K(q)$. Neste mesmo artigo R. Block classifica as representações irredutíveis de dimensão infinita da álgebra das matrizes de $2 \times 2 \mathrm{com}$ traço zero. Está é uma álgebra de Lie simples gerada por três elementos $e=\left(\begin{array}{ll}0 & 1 \\ 0 & 0\end{array}\right), f=\left(\begin{array}{ll}0 & 0 \\ 1 & 0\end{array}\right)$ e $h=\left(\begin{array}{cc}1 & 0 \\ 0 & -1\end{array}\right)$.

No caso da álgebra envelopante $U_{s}$ de $S l_{2}(K)$ os ideais primitivos estão dados por $I_{\lambda}=$ $U_{s}(Q-\lambda)$, onde $Q$ é o elemento de Casimir. Assim $U_{s} / I_{\lambda} \simeq A_{1}$, além disso o ideal $U_{s}(Q-\lambda)$ é um ideal maximal para $\lambda \in K, K$ um corpo algebricamente fechado de caraterística zero. Neste trabalho Block define o grau de um $\mathfrak{B}$-módulo irredutível, e dá uma caraterização explicita para os módulos de grau um, esta caraterização encontra-se na parte final do artigo (Block , 1981). Neste ordem, fazemos uma caraterização para os módulos de grau dois.

O estudo das representações irredutíveis da primeira álgebra de Weyl é dividida em os $A_{1}$-módulos de $S$-torção e os $A_{1}$-módulos livres de $S$-torção. Os primeiros são da forma $M(\lambda)=(K[p], \lambda)$ onde $p$ age como a multiplicação por $p$ e $q$ age como $\lambda-d / d p$, estes módulos foram classificados por Block em (Block, 1979). Para $A_{1}$-módulos livres de $S$ torção Block em (Block, 1981) mostrou que existe uma bijeção entre $A_{1}^{\Delta}(i r r)$ e $\mathfrak{B}^{\triangle}(i r r)$, a saber:

$$
\begin{gathered}
{[N] \in A_{1}^{\Delta}(i r r) \longrightarrow\left[S^{-1} N\right] \in \mathfrak{B}^{\Delta}(i r r) .} \\
{[M] \in \mathfrak{B}^{\Delta}(i r r) \longrightarrow\left[\operatorname{Soc}_{A_{1}}(M)\right] \in A_{1}^{\Delta}(i r r) .}
\end{gathered}
$$

Onde $A_{1}^{\Delta}(i r r)=\left\{[F] \mid F\right.$ é um $A_{1}$ - módulo irredutível e livres de $S$ - torção $\}$, e $[F]$; é formada por todos os $A_{1}$-módulos livres de $S$-torção isomorfos a $F$. Da mesma forma é definido 
o conjunto $\mathfrak{B}^{\triangle}(i r r)=\{[F] \mid F$ é um $\mathfrak{B}-$ módulo irredutível $\}$.

No capítulo 2, caraterizamos o $\operatorname{Soc}_{R}(M)$ para $M$ um $\mathfrak{B}$-módulo irredutível de grau dois como foi feito em (Block, 1981), para módulos de grau um, onde $R=A_{1}$ ou $R=\rho_{\lambda}\left(U_{s}\right)$. Denotamos por $\rho_{\lambda} U_{s}$ a imagem de $\rho_{\lambda}$, onde $\rho_{\lambda}: U_{s} \longrightarrow A_{1}$ é dado por $\rho_{\lambda}(e)=q$, $\rho_{\lambda}(f)=-(q p+\lambda+1) p$ e $\rho_{\lambda}(h)=2 q p+\lambda+1$.

O teorema para módulos de grau um enuncia-se como segue:

Teorema 1. Suponha $N$ um $\mathfrak{B}$-módulo irredutivel de grau um e $\lambda \in K$. Podemos identificar $N$ com $K(q)$ onde p.f $=t f+f^{\prime}$ e q.f $=q f$. Então

$$
\operatorname{Soc}_{A_{1}}(N)=K\left[q,\left(q-\alpha_{1}\right)^{-1}, \cdots,\left(q-\alpha_{l}\right)^{-1}\right]
$$

onde $\alpha_{i}$ são os polo de t. Além disso $\operatorname{Soc}_{\rho_{\lambda} U_{s}}(N)=\operatorname{Soc}_{A_{1}}(N)$ salvo que:

$$
\begin{array}{lrl}
v_{q}(t) & \geq 0 & \lambda \in \mathbb{Z} \\
v_{q}(t) & =-1 & \operatorname{Res}_{0}(t)=-\lambda
\end{array}
$$

Se temos (3), então $\operatorname{Soc}_{\rho_{\lambda} U_{s}} N=q^{-\lambda} \operatorname{Soc}_{A} N$. De (4) e supondo $\alpha_{l}=0$ temos

$$
\operatorname{Soc}_{\rho_{\lambda} U_{s}} N=K\left[q,\left(q-\alpha_{1}\right)^{-1}, \cdots,\left(q-\alpha_{l-1}\right)^{-1}\right]
$$

Se $M$ é um $\mathfrak{B}$-módulo irredutível de grau dois e $m \in M-\{0\}$, então existe $F_{1}=p^{2}+a p+b \in$ $\mathfrak{B}$ irredutível, preservado e com uma propriedade descrita no capítulo 2 tal que $F_{1} m=0$. Pode-se identificar a classe de $M([M])$ com a classe de $F_{1}\left(\left[F_{1}\right]\right)$, a classe dos polinômios irredutíveis em $\mathfrak{B}$ similares a $F_{1}=p^{2}+a p+b$, onde $a, b \in K(q)$.

Usando o polinômio $F_{1}$ damos a $K(q) \times K(q)$ estrutura de $\mathfrak{B}$-módulos, onde as ações de $p$ e $q$ estão dadas somo segue

$$
\begin{aligned}
& q(f, g)=(q f, q g) \\
& p(f, g)=\left(f^{\prime}-b g, f+g^{\prime}-a g\right)=\left(\left(\begin{array}{cc}
\frac{d}{d q} & -b \\
1 & \frac{d}{d q}-a
\end{array}\right)\left(\begin{array}{l}
f \\
g
\end{array}\right)\right)^{t} .
\end{aligned}
$$

Identificando $m \in M-\{0\}$ como $(1,0)(u=(1,0))$, da ação acima temos o isomorfismo $M \simeq K(q) \times K(q)$. Assim $F_{1}$ é anulador do elemento $(1,0) \in K(q) \times K(q)$, e portanto $K(q) \times K(q)$ é um $\mathfrak{B}$-módulo irredutível, isomorfo a $M$.

Sejam $N$ um $\mathfrak{B}$-módulo irredutível de grau dois, $F_{1}=p^{2}+a p+b \in \mathfrak{B}, \operatorname{Pol}\left(F_{1}\right)=$ $\left\{\alpha_{1}, \cdots, \alpha_{m}\right\}$ e $\lambda \in K$. Definimos $D=D^{+} \cup D^{-}$onde $D^{+(-)}=\left\{-\theta_{1},-\theta_{2}\right\}+\mathbb{Z}^{+(-)}$e 
$\theta_{1}, \theta_{2}$ são as raízes do polinômio $Q_{0, F_{1}}(\xi)$.

Do anterior, o principal resultado de tese enuncia-se como segue:

Teorema 2. Usando as notações anteriores temos o isomorfismo $N \simeq K(q) \times K(q)$, onde p e q agem como a multiplicação á esquerda pelas matrizes $\left(\begin{array}{cc}\frac{d}{d q} & -b \\ 1 & \frac{d}{d q}-a\end{array}\right)$ e $\left(\begin{array}{cc}q & 0 \\ 0 & q\end{array}\right)$ respectivamente. Então

$$
\operatorname{Soc}_{\rho(\lambda) U s} N=\operatorname{Soc}_{A_{1}} N
$$

salvo que:

$$
\begin{aligned}
& 0 \notin \operatorname{Pol}\left(F_{1}\right) \quad \text { e } \quad \lambda \notin \mathbb{Z}^{-}, \\
& 0 \in \operatorname{Pol}\left(F_{1}\right) \quad e \quad-\lambda \in D,
\end{aligned}
$$

Se temos (6), então $\rho(\lambda) U s\left(\begin{array}{l}1 \\ 0\end{array}\right)=q^{-\lambda} A_{1}\left(\begin{array}{l}1 \\ 0\end{array}\right)$. Se temos (7) existe $t \in \mathbb{N}$ tal que o módulo quociente

$$
q^{t} A_{1}\left(\begin{array}{l}
1 \\
0
\end{array}\right) / \rho(\lambda) U s\left(\begin{array}{l}
1 \\
0
\end{array}\right)
$$

é isomorfo com um dos seguintes módulos de Verma de $\mathrm{Sl}_{2}(\mathrm{~K})$,

1. $M(-\lambda-1)$.

2. $M(-\lambda-t)$, se $Q_{0, \gamma}(-\lambda-t)=0$ para $t \in \mathbb{N}$.

3. $M(-\lambda+t)$ e $W=q^{t} A_{1}\left(\begin{array}{l}1 \\ 0\end{array}\right)$, se $Q_{0, \gamma}(-\lambda+t)=0$ para $t \in \mathbb{N}$.

$\operatorname{Sejam} \Delta\left(F_{1}\right)=\left\{\alpha \in \operatorname{Pol}\left(F_{1}\right) \mid Q_{\alpha, F_{1}}(0)=0\right\}, \Delta^{\prime}\left(F_{1}\right)=\left\{\alpha \in \operatorname{Pol}(\alpha) \mid Q_{\alpha, F_{1}}(n) \neq 0, \forall n \in \mathbb{Z}\right\}$ e $K\left[q,\left(q-\alpha_{1}\right)^{-1}, \cdots,\left(q-\alpha_{l}\right)^{-1}\right] \times K\left[q,\left(q-\alpha_{1}\right)^{-1}, \cdots,\left(q-\alpha_{l}\right)^{-1}\right]=K_{P o l\left(F_{1}\right)} \times K_{P o l\left(F_{1}\right)}$. Logo $\operatorname{Pol}\left(F_{1}\right)=\Delta^{\prime}\left(F_{1}\right) \cup \Delta\left(F_{1}\right)$. Para o módulo $W=A_{1}\left(\begin{array}{l}1 \\ 0\end{array}\right)=\operatorname{Soc}_{A_{1}}(N)$ temos os seguintes resultados:

1. Se $\Delta\left(F_{1}\right)=\left\{\alpha_{1}, \cdots, \alpha_{j}\right\}$, do lema 23 , segue-se

$$
W \subset W_{1} \subset \cdots \subset W_{1 \cdots j+t}=K_{P o l\left(F_{1}\right)} \times K_{P o l\left(F_{1}\right)},
$$

sendo $W_{1 \cdots i}=A_{1}\left(\begin{array}{c}\prod_{k=1}^{i}(q-\alpha)^{-\theta_{\alpha_{k}}\left(F_{1}\right)-3} \\ 0\end{array}\right)$ e $\theta_{\alpha_{k}}\left(F_{1}\right)$ a menor raiz inteira positiva de $Q_{\alpha_{k}, F_{1}}(\xi)$ 
2. $K\left[q,(q-\beta)^{-1} \mid \beta \in \Delta^{\prime}\left(F_{1}\right)\right] \times K\left[q,(q-\beta)^{-1} \mid \beta \in \Delta^{\prime}\left(F_{1}\right)\right] \subset W$.

3. Se $\Delta_{0}\left(F_{1}\right)=\emptyset$, do lema 20 , segue-se que $W_{\alpha} / W$ é irredutível e de $S$-torção isomorfo a $M(\alpha)$.

4. Se $\Delta_{0}\left(F_{1}\right) \neq \emptyset$ para todo $\alpha \Delta_{0}\left(F_{1}\right)$ temos $\operatorname{dim}_{K}(T(\alpha))<\infty$ e $\nu_{\alpha}(a)=-1, \nu_{\alpha}(b) \geq-1$. Do lema $23, W_{\alpha} / W$ pertence ao $\operatorname{Ext}_{1}(M(\alpha), M(\alpha))$.

5. $\operatorname{Ext}_{1}(M(\alpha), M(\alpha))=M(\alpha) \oplus M(\alpha)$.

Afirmação 1. O $A_{1}$-módulo $W$ é irredutivel.

Teorema 3. $\Delta\left(F_{1}\right) \neq \varnothing$ se, e somente se, $\left(\begin{array}{l}1 \\ 0\end{array}\right)$ não gera $K_{P o l\left(F_{1}\right)} \times K_{P o l\left(F_{1}\right)}$. Ver (26).

Corolario 1. $K_{P o l\left(F_{1}\right)} \times K_{P o l\left(F_{1}\right)}$ é irredutivel se, e somente se, $\Delta\left(F_{1}\right)=\varnothing$. Além disso $W=K_{P o l\left(F_{1}\right)} \times K_{P o l\left(F_{1}\right)}$.

Afirmação 2. Se $\alpha \in \Delta\left(F_{1}\right)$, temos $W_{\alpha}=A_{1}\left(\begin{array}{c}(q-\alpha)^{-\theta_{\alpha}\left(F_{1}\right)-3} \\ 0\end{array}\right)$. Portanto, $W_{\alpha} / W e ́$ irredutivel de S-torção e isomorfo a $M(\alpha)$. Se $\nu_{\alpha}(a)=-1, \operatorname{dim}_{K}(T(\alpha))=-\operatorname{Res}_{\alpha}(a)$ e $\nu_{\alpha}(b) \geq-1$, então $V_{\alpha}=A_{1}\left(\begin{array}{c}(q-\alpha)^{-1} \\ 0\end{array}\right)$ é um $A_{1}$-submódulo de $W_{\alpha}$ e temos a cadeia de comprimento $2,0 \subset W \subset V_{\alpha} \subset W_{\alpha}$. O conjunto $T(\alpha)=\left\{p^{n}\left(\begin{array}{l}1 \\ 0\end{array}\right) \mid n \in \mathbb{N}\right\}$.

Corolario 2. $K_{P o l\left(F_{1}\right)} \times K_{P o l\left(F_{1}\right)}$ é cíclico gerado por $A_{1}\left(\begin{array}{c}\prod_{\alpha \in \Delta\left(F_{1}\right)}(q-\alpha)^{-\theta_{\alpha}\left(F_{1}\right)-3} \\ 0\end{array}\right)$ ede comprimento finito. 


\section{Sumário}

Resumo ..................................

Introdução . . . . . . . . . . . . . . . . . . . . . . . . vii

1 Preliminares $\quad 1$

1.1 Álgebra envelopante . . . . . . . . . . . . . . . . . . 1

1.2 Álgebra de operadores diferenciáveis . . . . . . . . . . . . . . . . . . 2

1.3 Álgebras de Lie nilpotentes e suas representações . . . . . . . . . . . . . . . 3

1.4 Polinômios irredutíveis sobre $\mathfrak{B} \ldots \ldots \ldots \ldots$

1.5 Módulos de Verma . . . . . . . . . . . . . . . . . . . . 9

$1.6 \quad S l_{2}$-módulos $\ldots \ldots \ldots \ldots \ldots \ldots$

1.6 .1 Álgebra envelopante de $S l_{2} \ldots \ldots \ldots \ldots$

1.6 .2 Ideais primitivos de $S l_{2}(K) \quad \ldots \ldots \ldots \ldots \ldots$

1.7 Representações irredutíveis . . . . . . . . . . . . . . . . . . . . . 14

2 Módulos de grau dois sobre a primeira álgebra de Weyl 19

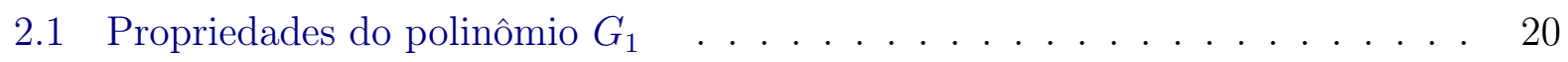

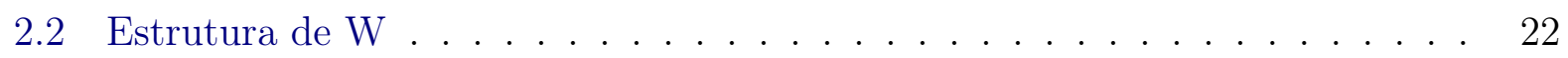

2.2 .1 Wé irredutivel . . . . . . . . . . . . . . . . . . 23

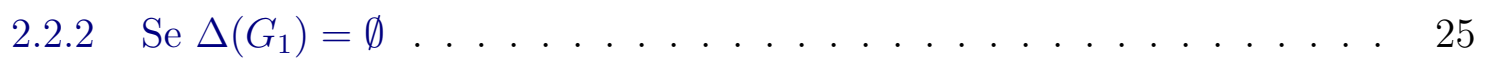

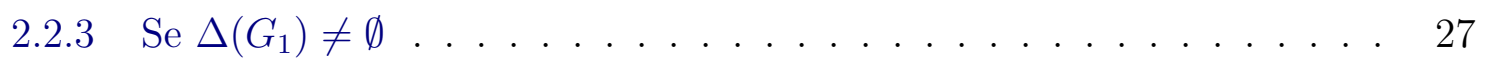

2.3 Relação entre os módulos $W$ e $K_{P o l\left(F_{1}\right)} \times K_{P o l\left(F_{1}\right)} \ldots \ldots \ldots \ldots$. . . . . 31

3 Módulos irredutíveis de grau dois

$\begin{array}{ll}\text { sobre } S l_{2}(K) & 37\end{array}$

3.0.1 Se $0 \notin \operatorname{Pol}\left(F_{1}\right) \ldots \ldots \ldots \ldots \ldots$

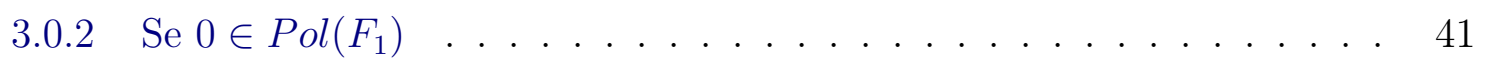

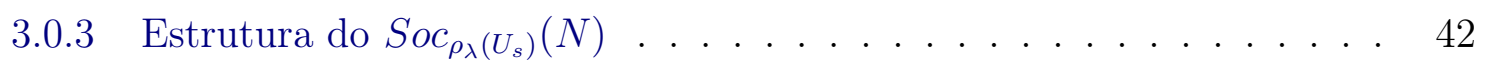

4 Extensões De Módulos irredutíveis $\quad 47$

4.1 Análise do caso $F \in A_{1}$ com $F$ redutível em $\mathfrak{B} \ldots \ldots$. . . . . . . . . . 49

$\begin{array}{ll}\text { Referências Bibliográficas } & 51\end{array}$ 


\section{Capítulo 1}

\section{Preliminares}

\section{$1.1 \quad$ Álgebra envelopante}

As definições e conceitos apresentados a continuação, podem ser consultadas com mais detalhe nos livros de (Jacobson , 1979) e (Humphreys , 1972). Tudo o apresentado neste capitulo referente as álgebras de Lie nilpotentes pode ser consulta nos trabalhos de Dixmier (Dixmier , 1963) e (Dixmier , 1977b).

Definição 1. Seja L uma álgebra de Lie. Dizemos que $(U, \pi)$ é a álgebra envelopande de L, se são satisfeitas as seguintes condições:

1. U é associativa.

2. $\pi: L \longrightarrow U$ é um homomorfismo de álgebras de Lie, e para cada álgebra associativa $V$ tal que existe um homomorfismo de álgebras de Lie $\varphi: L \longrightarrow V^{(-)}$, então existe um único homomorfismo $\psi$ de álgebras associativas que faz comutar o seguinte diagrama

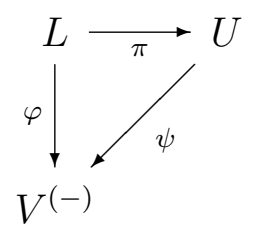

Da definição de álgebra envelopante, segue que $(U, \pi)$ é única. Denotaremos o par $(U, \pi)$ como $U(L)$.

Seja $L$ uma álgebra de Lie, definimos o seguinte produto tensorial

$$
T^{0}(L)=K, T^{1}(L)=L, \cdots, T^{m}(L)=\bigotimes_{i=1}^{m} L .
$$

Então a álgebra tensorial de $L$ é dada por

$$
T(L)=\bigoplus_{i=0}^{\infty} T^{i}(L)
$$

O produto em $T(L)$, é definido como

$$
\left(x_{1} \otimes \cdots \otimes x_{k}\right)\left(y_{1} \otimes \cdots \otimes y_{t}\right)=x_{1} \otimes \cdots \otimes x_{k} \otimes y_{1} \otimes \cdots \otimes y_{t} \in T^{k+t}(L) .
$$


De $T(L)$, podemos construir duas álgebras clássicas, a álgebra simétrica $\mathfrak{G}(L)$ e a álgebra envelopante universal $U(L)$. A álgebra $\mathfrak{G}(L)$ é definida como o quociente entre a álgebra tensorial e o ideal $I$ de $T(L)$, gerado pelos elementos $x \otimes y-y \otimes x$. Se fizemos $I_{m}=I \cap T^{m}(L)$, então $I=\bigoplus I_{m}$, obteremos os quocientes $S^{i}(L)=T^{i} / I_{i}$, assim $\mathfrak{G}(L)=\bigoplus S^{i}(L)$.

A álgebra $U(L)$ é definida como o quociente entre $T(L)$ é o ideal $J$ de $T(L)$, gerada pelos elementos $[x, y]-x \otimes y+y \otimes x$, assim temos

$$
U(L)=T(L) / J .
$$

Teorema 4. (teorema de $P B W$ )

Seja L uma álgebra de Lie, com base $X=\left\{x_{i} \mid i \in \mathbb{N}\right\}$, então o conjunto

$$
\left\{x_{i_{1}}^{n_{1}} \cdots x_{i_{t}}^{n_{t}} \mid x_{i_{j}} \in X \text { e } n_{i} \in \mathbb{N}\right\}
$$

é uma base da álgebra envelopante de $L$, para $i_{1}<\cdots<i_{t}$.

Sempre podemos substituir $\mathbb{N}$ por um conjunto totalmente ordenado $I$.

Demonstração. ver Jacobson, álgebras de Lie.

Teorema 5. Suponha $L=L_{1} \oplus L_{2}$, então $U(L)=U\left(L_{1}\right) \otimes U\left(L_{2}\right)$.

Teorema 6. A álgebra $U(L)$ é Noethreriana.

\section{2 Álgebra de operadores diferenciáveis}

Definição 2. Seja $R$ um anel e $D: R \longrightarrow R$ uma derivação, $D(a b)=D(a) b+a D(b)$, para todo par $a, b \in R$. Então o par $(R, D)$ é um anel diferenciável.

Definição 3. $\quad i$. Um módulo diferenciável, é um módulo sobre um anel diferenciável $R$, munido com uma aplicação $\delta: M \longrightarrow M$ tal que $\delta(a m)=D(a) m+a \delta(m)$ para todo $a \in R$ e $m \in M$.

ii. A aplicação $\delta$, é chamado operador diferenciável sobre $M$.

iii. Um ideal diferenciável de $R$, é um ideal de $R$ que tem estrutura de $R$-módulo diferenciável.

Exemplo 1. Sejam $K$ um corpo de característica zero e $R=K[x]\left[\partial_{x}\right]$, onde $\partial_{x}$ a derivação usual de polinômios na variável $x$. Então $R$ é um anel diferenciável.

Definição 4. Um homomorfismo $\varphi$, entre dois R-módulos diferenciáveis é uma aplicação

$$
\varphi:\left(M_{1}, D_{1}\right) \longrightarrow\left(M_{2}, D_{2}\right),
$$

tal que $\varphi$ é um homomorfismo de R-módulos e $\varphi D_{1}=D_{2} \varphi$.

Para $A_{n}=K\left[x_{1}, \cdots, x_{n}\right]$ junto com os operadores diferenciáveis $\partial_{1}, \cdots, \partial_{n}$, onde $\partial_{i} x_{j}-$ $x_{i} \partial_{j}=1$, temos a ação sobre o anel de polinômios $K\left[x_{1}, \cdots, x_{n}\right]$, dada pela ação de $x_{i}$ como a multiplicação por $x_{i}$ e $\partial_{j}$ como a derivação com respeito a $x_{j}$. 
Seja $P=\sum_{i=1}^{t} f_{i} \partial_{i}$ um operador linear em $A_{n}$, definimos $P(f)=\sum_{i}^{t} f_{i} \partial_{i} f$ para $f \in$ $K\left[x_{1}, \cdots, x_{n}\right]$. Se consideramos o núcleo do operador $P$ obtemos o conjunto

$$
\operatorname{ker}(P)=\left\{f \in K\left[x_{1}, \cdots, x_{n}\right] \mid \sum f_{\alpha} \partial_{\alpha}(f)=0\right\}
$$

O anel $K\left[x_{1}, \cdots, x_{n}\right]$ tem estrutura de $A_{n}$-módulo, e portanto definimos o módulo associado ao núcleo de $P$, como o módulo $A_{n} / A_{n} P$.

De forma geral, para $P_{1}, \cdots, P_{n}$ operadores em $A_{n}$, o módulo associado ao sistema

$$
P_{1}(f)=\cdots=P_{n}(f)
$$

está dado por o módulo cíclico $A_{n} /\left(\sum A_{n} P_{i}\right)$. Reciprocamente, para todo $A_{n}$-módulo irredutível $M$, temos um operador $S$ em $A_{n}$ tal que o quociente $A_{n} / A_{n} S$ é isomorfo a $M$, definindo $A_{n} S$ como o $A_{n}$-submódulo de $M$ gerado por $S$.

\section{3 Álgebras de Lie nilpotentes e suas representações}

Neste capitulo, apresentaremos algumas definições e resultados técnicos das álgebras de Weyl, que usaremos nos próximos capítulos. Sempre suporemos que as álgebras sobre $K$ são associativa, onde $K$ um corpo algebricamente fechado de característica zero.

Definição 5. Seja K um corpo de característica zero, e g uma álgebra de Lie. Dizemos que $g$ e nilpotente se $g^{n}=0$ para algum $n \in \mathbb{N}$, onde $g^{2}=[g, g]$ e $g^{k}=\left[g^{k-1}, g\right]$.

Definição 6. Seja K um corpo de característica zero, a enésima álgebra de Weyl $A_{n}$, é definida como o anel de polinômios $K\left[x_{1}, \cdots, x_{n}, y_{1}, \cdots, y_{n}\right]$ mundo com a relação $x_{i} y_{j}-y_{j} x_{i}=\delta_{i j}$. No caso $n=1$, temos $A_{1}=K[q][p]$, onde $p q-q p=1$.

Seja $A$ uma álgebra sobre $K$. Uma filtração de $A$ é um conjunto $\left\{A_{i}\right\}_{i \in \mathbb{N}}$ de sub-espaços de $A$ tal que $A_{i} . A_{j} \subseteq A_{i+j}$ e $A=\bigcup A_{i}$. Para a primeira álgebra de Weyl temos a filtração

$$
A_{1}^{n}=\sum_{i+j \leq n} k q^{i} p^{j}
$$

Seja $A$ uma álgebra sobre $K$. Uma graduação de $A$ é um conjunto $\left\{A_{i}\right\}_{i \in \mathbb{N}}$ tal que

$$
A_{i} \cdot A_{j} \subseteq A_{i+j} \text { e } A=\oplus A_{i}
$$

Do trabalho de Dixmier (Dixmier , 1963) temos o seguinte lema.

Lema 1. Sejam $i, j, s, t$ inteiros não negativos. Então

$$
\left(q^{i} p^{s}\right)\left(q^{j} p^{t}\right)=\sum \alpha_{k} q^{i+j-k} p^{s+t-k}
$$

Em virtude do lema anterior, para a primeira álgebra de Weyl obtemos a graduação

$$
A_{1}^{n}=\sum_{i-j=n} k q^{i} p^{j}
$$


Outra forma de definir as álgebras de Weyl, é dada em (McConnell e Robson , 1987). Seja $R=K\left[x_{1}, \cdots, x_{n}\right]$, definimos por indução o anel de polinômios torcidos

$$
R_{0}=R, R_{i}=R_{i-1}\left[y_{i}, \frac{\partial}{\partial x_{i}}\right], \quad i=1, \cdots, n .
$$

Pelo teorema das bases Hilbert $R_{n}$ é Noetheriano, além disso temos em $R_{n}$ a relação

$$
x_{i} y_{j}=y_{j} x_{i}+\frac{\partial x_{i}}{\partial x_{j}}=y_{j} x_{i}+\delta_{i j}
$$

então existe um conjunto gerador de $R_{n}$, que satisfaz a definição da álgebra de Weyl. Da mesmo forma existe um conjunto de geradores da álgebra de Weyl $A_{n}$, que satisfaz a definição do anel de polinômios torcidos nas variáveis $x_{i}, y_{i}$.

Teorema 7. $A_{n}$ é simples (i.e. não tem ideais bilaterais) e Noetheriano.

Demonstração. Pela parte anterior $A_{n}$ é Noetheriano. Seja $I$ um ideal não nulo de $A_{n}$. Para $0 \neq a \in I, a=\sum \lambda_{\alpha \beta} x^{\alpha} y^{\beta}$ com $\alpha, \beta \in \mathbb{N}^{n}$. Suponhamos que $\alpha=\left(\alpha_{1}, \cdots, \alpha_{n}\right), \beta=$ $\left(\beta_{1}, \cdots, \beta_{n}\right), \bar{\alpha}_{i}=\alpha=\left(\alpha_{1}, \cdots, \alpha_{i-1}, \alpha_{i+1}, \cdots, \alpha_{n}\right)$, então

$$
\begin{aligned}
x_{i} a-a x_{i} & =\sum \lambda_{\alpha \beta} x^{\alpha_{i}+1} x^{\bar{\alpha}_{i}} y^{\beta}+\sum \lambda_{\alpha \beta} x^{\alpha}\left(x_{i} y^{\beta}+\frac{\partial y^{\beta}}{\partial y_{i}}\right) \\
& =\sum \lambda_{\alpha \beta} x^{\alpha} \frac{\partial y^{\beta}}{\partial y_{i}}=\frac{\partial a}{\partial y_{i}} .
\end{aligned}
$$

Dado que $y_{i} a-a y_{i}=\frac{\partial a}{\partial x_{i}}$, então existe $0 \neq b \in k$ tal que $b \in I$, então $A_{n}$ é simples.

Seja $g$ uma álgebra de Lie nilpotente e $U(g)$ sua álgebra envelopante. O estudo das representações de $g$ é equivalente ao estudo das representações de $U(g)$. Este fato é valido em geral para qualquer álgebra de Lie. Definimos o centro de $U(g)$ como $Z$, e $C(g)$ como o comutador de $g$ em $U(g)$, isto é, $C(g)$ é o conjunto dos elementos $x \in U(g)$ tais que $[g, x]=0$. J. Dixmier em (Dixmier , 1963), da uma relação ente as álgebras de Lie nilpotentes e as álgebras de Weyl.

Definição 7. Seja g uma álgebra de Lie nilpotente. Dizemos que um K-espaço vetorial $V$ é um g-módulo, se existe uma aplicação bilinear $\tau: g \times V \longrightarrow V$ tal que $\tau([x, y], v)=$ $\tau(x y, v)-\tau(y x, v)$, para cada $x, y \in g$ e $v \in V$.

Definição 8. Seja g uma álgebra de Lie e $V$ um $K$-espaço vetorial. Dizemos que $\rho: g \longrightarrow$ $g l(V)$ é uma representação de $g$, se $\rho$ é uma transformação linear e $\rho([x, y]) v=[\rho(x), \rho(y)] v$, para cada $x, y \in g$ e $v \in V$, sendo $g l(V)$ a álgebra das transformações lineares de $V$ em $V$. Se $V$ é um g-módulo irreductivel a representação $\rho$ diz-se irredutivel.

Definição 9. Seja g uma álgebra de Lie nilpotente. Dizemos que um ideal bilateral I não trivial de $U(g)$ e primitivo, se existir um módulo irredutivel $M$ tal que $I M=0$.

Teorema 8. (Dixmier, 1963)) Sejam V um espaço vetorial de dimensão menor o igual que $\aleph_{0}$ sobre $\mathbb{C}, A \subset \operatorname{End}(V)$ uma subálgebra irredutivel e $A^{\prime}$ os elementos que comutam com $A$ em $\operatorname{End}(V)$, então $A^{\prime}=\mathbb{C}$. 
Demonstração. Pelo lema de Schur, $A^{\prime}$ é um corpo contendo $\mathbb{C}$ (ver (Dixmier , 1977b), pág. 133). Se $x \in A^{\prime}$ e $x \notin \mathbb{C}$, então $x$ é transcendente sobre $\mathbb{C}$, portanto $A^{\prime}$ contem um corpo isomorfo a $\mathbb{C}(x)$. Para cada $\lambda \in \mathbb{C}$, os elementos $(x-\lambda)^{-1}$ são linearmente independentes sobre $\mathbb{C}$ e consequentemente $\operatorname{dim}_{\mathbb{C}} \mathbb{C}(x)>\aleph_{0}$. Por outra parte, segue que $(x-\lambda)^{-1}$ são linearmente independentes sobre $\mathbb{C}$, para cada $\lambda \in \mathbb{C}$. Logo $\operatorname{dim}_{A^{\prime}} V \geq \operatorname{dim}_{\mathbb{C}} A^{\prime} \geq \operatorname{dim}_{\mathbb{C}} C(x)>\aleph_{0}$.

Lema 2. (Dixmier, 1963) Seja $B$ uma álgebra sobre $K, I$ um ideal bilateral de $A_{1} \otimes B$ e $J=I \cap B$, então $I=A_{1} \otimes J$.

Demonstração. Para cada $c=\sum p^{i} q^{j} \otimes b_{i j} \in I$, definimos

$$
v(c)=\max _{(i, j)}\left\{i+j \mid b_{i j} \neq 0\right\} .
$$

Se $v(c)=0$, então $c=1 \otimes b \in A_{1} \otimes J$. Suponha que $v(c)<n$, então $b_{i j} \in J$. Por outra parte $[p, c] \in I \mathrm{e}$

$$
[p, c]=\sum p^{i+1} q^{j} \otimes b_{i j}+\sum p^{i} q^{j} p \otimes b_{i j}=\sum j p^{i} q^{j-1} \otimes b_{i j}
$$

$\operatorname{logo} b_{i j} \in J$, para $j \neq 0$. Substituindo $p$ por $q$ obtemos analogamente que $b_{i j} \in J$ para $i \neq 0$. Dado que $1 \otimes b_{00}=c-\sum_{i+j>1} p^{i} q^{j} \otimes b_{i j}$ e $\sum_{i+j>1} p^{i} q^{j} \otimes b_{i j} \in A_{1} \otimes J$, portanto $1 \otimes b_{00} \in I$ e $1 \otimes b_{00} \in J$.

Lema 3. (Dixmier, 1963) Sejam $A$ uma $K$-álgebra, $B$ e $C$ duas subálgebras de $A$, que geram $A$ e comutam. Se $B$ é isomorfo a $A_{1}$, então o morfismo canônico $\phi$ de $B \otimes C$ em $A$ é um isomorfismo.

Demonstração. Seja $\phi$ a aplicação canónica de $B \otimes C$ em $A$ determinada por $\phi(b \otimes c)=b c$, como $\operatorname{ker}(\phi)$ é um ideal de $B \otimes C$, segue-se que $\operatorname{ker}(\phi)=B \otimes J$, onde $J$ é um ideal de $C$. Note que $\phi(1 \otimes c)=c$ para cada $c \in C$, temos $J=0$. Portanto, $\phi$ é um isomorfismo.

Usando o lema anterior e indução mostra-se que $A_{n}$ é isomorfo a $\otimes_{K} A_{1} n$-vezes, já que o homomorfismo canônico e de fato um isomorfismo de $K$-álgebras.

Lema 4. (Dixmier, 1963) Seja g uma álgebra de Lie nilpotente, de dimensão maior que 2. Então existe um ideal $I \subset g$ com $\operatorname{dim} I=\operatorname{dim} g-1$ tal que $\operatorname{dim} Z(I)>1$, onde $Z(I)$ denota o centro de $I$.

Demonstração. Seja $g_{0}=g$. Como $g_{0}$ é nilpotente, $g^{\prime} \subset g_{0}$. Seja $\overline{g_{1}} \triangleleft g / g^{\prime}$ ideal de $g$ tal que $\operatorname{dim} g / g_{1}=1 \mathrm{e}\left[g, g_{1}\right] \subset g_{1}$. No seguinte passo, tomamos um ideal $\overline{g_{2}}$ do cociente $g_{1} /\left[g, g_{1}\right]$ tal que $\operatorname{dim} g_{1} / g_{2}=1$, já que $g_{1} /\left[g, g_{1}\right]$ resulta abeliano não nulo. Se $g_{1}=\left[g, g_{1}\right]$, então $g_{1}=g^{\prime}$, por um argumento indutivo $g^{n}=g_{1}$, em consequência $g$ não é nilpotente de dimensão finita, assim temos

$$
0=g_{n} \subset g_{n-1} \subset \cdots \subset g_{1} \subset g_{0}=g
$$

tal que $\operatorname{dim} g_{i} / g_{i+1}=1$ e cada $g_{i}$ é ideal de $g$. Seja $\{a, b\}$ uma base de $g_{n-2}$ tal que $b \in g_{n-1}$, então para cada $x \in g$ a representação adjunta $\left.a d_{g} x\right|_{g_{n-2}}$ é dada por, $\left.a d_{g} x\right|_{g_{n-2}}(a)=\lambda(x) a \mathrm{e}$ $\left.a d_{g} x\right|_{g_{n-2}}(b)=0$, assim temos a representação matricial

$$
\left(\begin{array}{cc}
0 & 0 \\
\lambda(x) & 0
\end{array}\right) .
$$


É claro que $\left.\lambda\right|_{g_{n-1}}=0$, e se $[a, b] \neq 0$ então $[a, b]=t b$. Logo $\left(g_{n-2}\right)^{n}=g_{n-1}$ para todo $n \in \mathbb{N}$. Se $\lambda=0$, então $g_{n-2} \subset Z(g)$. Se $\lambda \neq 0$, então para a representação $\rho: g \longrightarrow g l\left(g_{n-2}\right)$ dada por $\rho(x)=\left.a d_{g} x\right|_{g_{n-2}}$, temos $\operatorname{dim} \rho(g)=1$. Assim $\operatorname{ker}(\rho)=\mathfrak{h}$ é um ideal de $g \operatorname{com} g_{2} \subset Z(\mathfrak{h})$.

Se $\operatorname{dim} g>2$ e $\operatorname{dim} Z(g)=1$ então existem dois ideais $\mathfrak{h}, I$ de $g$ com as seguintes propriedades

1. $\operatorname{dim}(g / \mathfrak{h})=1, \operatorname{dim} I=2$.

2. $Z(g) \subset I \subset \mathfrak{h} \subset g$.

3. $Z(\mathfrak{h})=I$.

Introduziremos algumas notações que precisamos no decorrer do trabalho. Seja $x \in g-\mathfrak{h}$ e $y \in I-Z(g)$, então $[x, y] \in Z(g)$, de outra forma $g$ não é nilpotente. Então a álgebra $N$ gerada por $x, y, z=[x, y]$ é nilpotente de dimensão 3 .

Lema 5. (Dixmier, 1963) Sejam $U=U(g), U^{\prime}=U(\mathfrak{h}), V=U(N), Y=U(y), J$ o ideal gerado por $z+1$ e $W=N_{U^{\prime}}(J)$ ( o normalizador de $J$ em $U^{\prime}$ ). Seja $\varphi$ o homomorfismo canônico de $U$ em $U / J$. Então

1. $\varphi(V) \cong A_{1}$.

2. $\varphi(U)=\varphi(V) \otimes \varphi(W)$.

3. $\varphi\left(U^{\prime}\right)=\varphi(Y) \otimes \varphi(W)$.

4. $\varphi(Y) \cong K[y]$.

5. $\varphi(W)$ é isomorfo a uma álgebra quociente de $\varphi\left(U^{\prime}\right)$.

Demonstração. 1. Como $[\varphi(x), \varphi(y)]=1$ e $\varphi(V)$ é gerado por $\varphi(x), \varphi(y)$, e em consequência $\varphi(V)$ é isomorfo a $A_{1}$.

2. Para a demonstração de (2), (3), mostraremos que $V, W(Y, W)$ geram $U\left(U^{\prime}\right)$ respectivamente. Fixando $a \in U^{\prime}$, a nilpotência de $g$ permite definir $v(a)$ como o inteiro $t$ tal que $a d^{t+1}(x) a=0$ e $a d^{t}(x) a \neq 0$, usaremos indução em $t$.

Se $t=0$ então $a \in W$. Suponhamos que a afirmação é valida para $v(a)<t$. Definimos $a_{i}=a d^{i}(x) a$, como $a d(x)$ é uma derivação e $z$ está no centro de $g$, temos

$$
\begin{aligned}
& a d(x)\left[a+a_{1} y+\cdots+\frac{a_{t}}{t !} y^{t}\right] \\
& =a d(x) a+a d(x) a_{1} y+a_{1} a d(x) y+\cdots+\frac{a d(x) a_{t-1}}{(t-1) !} y^{t-1}+\frac{a_{t}}{t !} t y^{t-1} z \\
& =a_{1}+a_{2} y+a_{1} z+\cdots+\frac{a_{t}}{(t-1) !} y^{t-1}+\frac{a_{t}}{(t-1) !} y^{t-1} z \\
& =(z+1)\left(a_{1}+a_{2} y+\cdots+\frac{a_{t}}{(t-1) !} y^{t-1}\right) .
\end{aligned}
$$

Pela hipótese, $a_{i}$ está na álgebra gerada por $Y$ e $W$, pois $v\left(a_{i}\right)<t$ para $i>0$. Por outra parte $a+a_{1} y+\cdots+\frac{a_{t}}{t !} y^{t} \in W$. Logo, a está na álgebra gerada por $Y, W$, e esta 
álgebra coincide com $U$, além disso $V, W$ geram $U$ pois $x \in V$.

Do anterior e de $(1,3)$ temos $(2)$.

3. Por (1), a imagem de $\varphi(Y) \otimes \varphi(W)$ é $\varphi\left(U^{\prime}\right)$. A sobrejectividade segues-se da definição do homomorfismo.

4. Segue-se definição de álgebra envelopante.

5. Considere a aplicação $\phi: \varphi(y) \times \varphi(W) \longrightarrow \varphi(W)$, dada por $\phi\left(y^{i}, b\right)=z^{i} b$.

Lema 6. (Dixmier, 1963) Com as hipóteses e notações anteriores, seja I um ideal bilateral de $U$ tal que $z+1 \in I$.

1. Existe um ideal bilateral $J$ de $\varphi(W)$ tal que $\varphi(I)=\varphi(V) \otimes J$. A álgebra $U / I$ é isomorfa a álgebra $A_{1} \otimes(\varphi(W) / J)$.

2. Seja $Y^{+}$a coleção dos elementos de $Y=K[y]$ que não têm parte constante, e $L=$ $\varphi\left(Y^{+}\right) \otimes \varphi(W)+\varphi(Y) \otimes J$, então L é um ideal bilateral de $\varphi\left(U^{\prime}\right)$. Se $I^{\prime}$ é a imagem reciproca de $L$ contida em $U^{\prime}$ então $U^{\prime} / I^{\prime} \cong \varphi(W) / J$.

Demonstração. 1. Pelas considerações feitas anteriormente existe um ideal bilateral $I$ de $\varphi(W)$ tal que $I=\varphi(V) \otimes I$. Como $z+1 \in I$ temos $J \subset I$, além disso $I / J$ é um ideal bilateral de $U / J$. Via o homomorfismo canônico $\tau: U \longrightarrow \varphi(U) / \varphi(I)$, definido como $\tau(a)=\varphi(a)+\varphi(I)$, temos o isomorfismo $U / I \cong \varphi(U) / \varphi(I)$.

$$
\begin{aligned}
U / I \cong \varphi(U) / \varphi(I) & \cong(\varphi(V) \otimes \varphi(W)) /(\varphi(V) \otimes \varphi(K)) \\
& \cong \varphi(V) \otimes \varphi(W / K) \cong A_{1} \otimes \varphi(W / K) .
\end{aligned}
$$

2. $U^{\prime} / I^{\prime} \simeq \varphi\left(U^{\prime}\right) / L \simeq\left(\varphi(Y) / \varphi\left(Y^{+}\right)\right) \otimes(\varphi(W) / K) \simeq \varphi(W) / K$.

Teorema 9. (Dixmier, 1963) Sejam K um corpo comutativo de característica zero, g uma álgebra de Lie nilpotente, com álgebra envelopante $U(g)$ e I um ideal bilateral de $U(g)$. Para $K$ algebricamente fechado, as seguintes afirmações são equivalentes

1. $Z(U(g) / I)=K$.

2. $U(g) / I$ é isomorfa a uma das álgebras $A_{p}$.

3. I é um ideal bilateral maximal de $U(g)$.

4. I é um ideal bilateral primitivo de $U(g)$.

Demonstração. Usamos indução na $\operatorname{dim}(g)$, no seguinte ordem

$$
(2) \Rightarrow(3) \Rightarrow(4) \Rightarrow(1) \Rightarrow(2)
$$

Em qualquer álgebra, independente da dimensão temos $(3) \Rightarrow(4)$, e $(2) \Rightarrow(3)$ se segue do fato que, $A_{p}$ é simples. 
Seja $\rho$ a representação que induz $I, \rho(U(g)) \simeq U(g) / I$. Substituindo $V$ por $\rho(U(g))$ no Teorema 8 concluímos que $(4) \Rightarrow(1)$.

Só resta mostrar $(1) \Rightarrow(2)$. Se $\operatorname{dim}(g)=1$ e $Z(U / I)=K$ então $U / I=K, \operatorname{logo}(1) \Rightarrow(2)$. Suponha a afirmação valida para $\operatorname{dim}(g)<n$. Seja $Z_{0}=I \cap Z(g)$.

Suponhamos que $Z_{0} \neq 0$. Sejam $L=g / Z_{0}, J$ o ideal bilateral gerado por $Z_{0}$ em $U(g)$ e $U(L)=U(g / K)$, do homomorfismo que induz $\tau: g \longrightarrow U(L)$ definido por $\tau(x)=\overline{(x)}$, o ideal $I_{Z_{0}}=I / Z_{0}$ é um ideal bilateral de $U(L)$.

$$
U / I \simeq\left(U(L) / U\left(Z_{0}\right)\right) /\left(I / U\left(Z_{0}\right)\right) \simeq U(L) / I_{Z_{0}}
$$

Então o centro de $U(L) / I_{Z_{0}}$ é $K$, da hipótese de indução temos o isomorfismo

$$
U(L) / I_{Z_{0}} \simeq A_{p}
$$

Suponhamos que $Z_{0}=0$. Como $g$ é nilpotente, $\operatorname{dim}(Z(g)) \geq 1$. De $Z(U / I)=K$, segue-se que $I \cap K=I \cap Z(g)=0$ e $I \cap(K+Z) \neq 0$. Do lema 6 , existe um ideal bilateral $I^{\prime}$ de $U^{\prime}$ tal que $U / I \simeq A_{1} \otimes\left(U^{\prime} / I^{\prime}\right)$. Como $Z(U / I)=K$, então $Z\left(U^{\prime} / I^{\prime}\right)=K$. Pela hipótese de indução existe $A_{p}$ tal que $U / I \simeq A_{1} \otimes A_{p} \simeq A_{p+1}$.

\subsection{Polinômios irredutíveis sobre $\mathfrak{B}$}

Seja $S=K[q]-\{0\}$. O domínio $\mathfrak{B}=S^{-1} A_{1}=K(q)[p]$, é um dominio de ideais principais, e portanto existe uma correspondência bijetora entre os elementos irredutíveis e os ideais maximais de $\mathfrak{B}$. Todo $\mathfrak{B}$-módulo irredutível $N$ e isomorfo a $\mathfrak{B} / \operatorname{ker}(\varphi)$, onde $\operatorname{ker}(\varphi)$ é um ideal máximal de $\mathfrak{B}$, portanto existe $H \in \mathfrak{B}$ tal que $\operatorname{ker}(\varphi)=H n$, para algum $0 \neq n \in N$. Todo os resultados desta seção são do trabalho feito por Block em (Block , 1981).

Dado um par de polinômios $f, g \in \mathfrak{B}$ não nulos, definimos o mínimo múltiplo comum à direita como o polinomio mônico não nulo $h(x)$ de menor grau tal que

$$
h=f_{1} f=g_{1} g .
$$

Definição 10. Dois polinômios $f, g \in \mathfrak{B}$ não nulos, são relativamente primos a direita, se para todos os polinômios $f_{1}, g_{1}, d \in \mathfrak{B}$ tal que $f=f_{1} d$ e $g=g_{1} d$ implica que dé unidade. De forma análoga definimos polinômios relativamente primos à esquerda.

Definição 11. Dois polinômios $f, g \neq 0$, são similares se existem polinomios $u, v$ não nulos tais que $f u=v g$, onde $f, v$ são relativamente primos á esquerda, e g, u são relativamente primos à direita.

Teorema 10. Dois polinômios de grau um $p+x_{0}, p+x_{1} \in \mathfrak{B}$ são similares, se e somente se, a diferença dos termos constantes $x_{0}-x_{1}$, é uma derivação logarítmica.

Demonstração. Suponhamos que $x-a$ similar com $x-b$. Então existe $c, d \in K[x]$ tais que $c(x-a)=(x-b) d, \log \mathrm{c}=d$ é $a-b=\frac{c^{\prime}}{c}$. 


\subsection{Módulos de Verma}

Seja $L$ uma álgebra de Lie semisimples de dimensão finita e $H$ uma sub-álgebra de Cartan, então

$$
L=H \oplus \bigoplus_{\alpha} L_{\alpha}
$$

Onde $L_{\alpha}$ é o espaço de peso $\alpha$ de $L$ relativos a subálgebra de Cartan de $H$. Definimos $\pi=\left\{\alpha \in H^{*} \mid L_{\alpha} \neq 0\right\}$, então $\pi$ é um sistema de raízes, com descomposição $\phi=\phi^{-} \cup \phi^{+}$, onde $\pi^{+}$é o conjunto das raízes positivas e $\pi^{-}$é o conjunto das raízes negativas. Escrevendo $N^{-}\left(^{+}\right)=\bigoplus_{\alpha \in \phi^{-}(+)} L_{\alpha}$, temos a subálgebra de Borel $\mathcal{B}=H \oplus N^{+}$. Além disso $L=N^{+} \oplus \mathcal{B}$ e $U(L)=U\left(N^{+}\right) U(\mathcal{B})$.

De (1.1), $L$ tem uma base dada por $h_{\alpha} \in H, e_{\alpha} \in N^{+}$e $f_{\alpha} \in N^{-}$, para cada $\alpha \in \pi$. Para $\lambda \in H^{*}$, definimos o ideal $I_{\lambda}$ de $U(L)$ como $I_{\lambda}=\sum U(L) e_{\alpha}+\sum U(L)\left(h_{\alpha}-\lambda\left(h_{\alpha}\right)\right)$. Usando o ideal $I_{\lambda}$ obtemos o módulo de Verma

$$
M(\lambda)=U(L) / I_{\lambda}
$$

Uma construção equivalente para o módulo de Verma é dada por

$$
M(\lambda)=U(L) \bigotimes_{U(\mathcal{B})} \mathbb{C}_{\lambda}
$$

O $U(\mathcal{B})$-módulo, $\mathbb{C}_{\lambda}$ está determinado pelas ações; $h .1=\lambda(h)$ e $e_{\alpha} \cdot 1=0$, assim $M(\lambda)$ tem estrutura de $H$-módulo. Para cada $\mu \in H^{*}$ definimos o subespaço

$$
M(\lambda)_{\mu}=\{x \in M(\lambda) \mid h(x)=\mu(h) x, \forall h \in H\} .
$$

Dizemos que $M(\lambda)_{\mu}$ é um espaço peso com peso $\mu$, se $M(\lambda)_{\mu} \neq 0$.

Teorema 11. $M(\lambda)=\bigoplus_{\mu \in H^{*}} M(\lambda)_{\mu}$

Demonstração. Sejam $\phi^{+}=\left\{\beta_{1}, \cdots, \beta_{n}\right\}$ e $m_{\lambda}=1+I_{\lambda}$, então o conjunto $f_{\beta_{1}}^{r_{1}} \cdots f_{\beta_{n}}^{r_{n}} m_{\lambda}$ é uma base para $M(\lambda)$. Para $h \in H$ temos

$$
\begin{aligned}
h f_{\beta_{1}}^{r_{1}} \cdots f_{\beta_{n}}^{r_{n}} m_{\lambda} & =-\beta_{1}(h) f_{\beta_{1}} h f_{\beta_{1}}^{r_{1}-1} \cdots f_{\beta_{n}}^{r_{n}} m_{\lambda}+f_{\beta_{1}} h f_{\beta_{1}}^{r_{1}-1} \cdots f_{\beta_{n}}^{r_{n}} m_{\lambda} \\
& =\left(\lambda-r_{1} \beta_{1}-\cdots-r_{n} \beta_{n}\right)(h) f_{\beta_{1}}^{r_{1}} \cdots f_{\beta_{n}}^{r_{n}} m_{\lambda} .
\end{aligned}
$$

Isto implica que $f_{\beta_{1}}^{r_{1}} \cdots f_{\beta_{n}}^{r_{n}} m_{\lambda} \in M_{\mu}$, para $\mu=\lambda-r_{1} \beta_{1}-\cdots-r_{n} \beta_{n}$ e

$$
M(\lambda)=\sum M(\lambda)_{\mu}
$$

Considere os funcionais $\mu_{i}-\mu$. Como $H$ não pode ser escrito como a união finita de subespaços de menor dimensão $\left(\operatorname{ker}\left(\mu_{i}-\mu\right)\right)$, então existe $h_{0} \in H$ tal que $\mu\left(h_{0}\right) \neq \mu_{i}\left(h_{0}\right)$ para todo $i$, além disso existem polinômios $p(x), q(x)$, com a propriedade

$$
p(x)\left(x-\mu\left(h_{0}\right)\right)+q(x) \prod_{i=1}^{t}\left(x-\mu_{i}\left(h_{0}\right)\right)=1 .
$$


Se $m \in M(\lambda)_{\mu} \cap\left(\sum_{i=1}^{t} M(\lambda)_{\mu_{i}}\right)$ e os $\mu_{i}$ são todos distintos, então $m=\sum m_{i}$ onde $(h-$ $\left.\mu_{i}(h)\right) m_{i}=0$, para cada $i$ e para cada $h \in H$, portanto $\prod_{i=1}^{t}\left(h-\mu_{i}(h)\right) m=0$ e

$$
0=p\left(h_{0}\right)\left(h_{0}-\mu\left(h_{0}\right)\right) m+q\left(h_{0}\right) \prod_{i=1}^{t}\left(h_{0}-\mu_{i}\left(h_{0}\right)\right) m=m .
$$

Teorema 12. $M(\lambda)$ tem um único submódulo maximal.

Demonstração. Seja $V$ um submódulo não nulo de $M(\lambda)$ tal que $V \neq M(\lambda)$, então para $v \in V$ não nulo, temos $v=\sum v_{\mu_{i}}$, onde $v_{\mu_{i}} \in M(\lambda)_{\mu_{i}}$. Se $h \in H$ temos

$$
\prod_{j, j \neq i}\left(h-\mu_{j}(h)\right) v=\prod_{j, j \neq i}\left(h-\mu_{j}(h)\right) v_{\mu_{i}}=\prod_{j, j \neq i}\left(\mu_{i}(h)-\mu_{j}(h)\right) v_{\mu_{i}}
$$

então $v_{\mu_{i}} \in V$ e $V=\bigoplus_{\mu} V_{\mu}$, assim todo submódulo é soma de submódulos de peso.

Como $V_{\lambda} \subset M(\lambda)_{\lambda}$ e $\operatorname{dim} M(\lambda)_{\lambda}=1$, então $V_{\lambda}=M(\lambda)_{\lambda}$, o que é absurdo pela hipóteses. Portanto, todo submódulo de $M(\lambda)$ está contido em $W=\sum_{\mu, \mu \neq \lambda} M(\lambda)_{\mu}$, assim $W$ é o único submódulo maximal de $M(\lambda)$.

\section{6 $S l_{2}$-módulos}

Nesta seção introduzimos os ideais primitivos das álgebras de Lie nilpotentes e de $S l_{2}(K)$. Além disso damos uma breve descrição dos quocientes de tipo $U(g) / I$, onde $g S l_{2}(K)$.

\subsection{1 Álgebra envelopante de $S l_{2}$}

Seja $K$ um corpo de característica zero. Definimos a álgebra de Lie $S l_{2}(K)$ como a álgebra gerada pelos elementos $e, f, h$, onde o produto de Lie é dado por

$$
[e, h]=h, \quad[h, e]=2 e,[h, f]=-2 f .
$$

Denotámos por $Z(g)$, o centro da álgebra envelopante $U(g)$ de $g=S l_{2}(K)$. Definimos $U(g)_{2 s}$, como o subespaço de $U(g)$ gerado pelos elemento $f^{k} h^{j} e^{i}$ tal que $k-i=s$, logo

$$
U(g)=\bigoplus_{s \in \mathbb{Z}} U(g)_{s}
$$

Observação 1. $U(g)_{0}$ é gerado pelos elementos $h, Q$, onde $Q=(h+1)^{2}+4 f e$, é o elemento de Casimir.

Teorema 13. $Z(g)=K[Q]$. Em particular $Z(g)$ é isomorfa ao anel de polinômios na variável $x, K[x]$.

Demonstração. Como $Q \in Z(g)$ temos $K[Q] \subset Z(g)$. Por outro lado $Z(g) \subset U(g)_{0}$ e portanto todo elemento $a \in Z(g)$ pode-se escrever da forma

$$
a=\sum a_{i}(h) Q^{i}
$$


onde $[e, a]=0$ e $h e=e h+2 e=e(h+2)$. De $h^{n-1} e=e(h+2)^{n-1}$ temos

$$
\begin{aligned}
h^{n} e=h^{n-1}(e h+2 e) & =h^{n-1} e h+2 h^{n-1} e \\
& =e(h+2)^{n-1} h+2 e(h+2)^{n-1} \\
& =e(h+2)^{n},
\end{aligned}
$$

$\operatorname{logo}\left[e, \sum a_{i}(h) Q^{i}\right]=\sum Q^{i}\left(e a_{i}(h)-a_{i}(h) e\right)=e \sum Q^{i}\left(a_{i}(h)-a_{i}(h+2)\right) \mathrm{e}$

$$
\sum Q^{i}\left(a_{i}(h)-a_{i}(h+2)\right)=0
$$

Portanto, $a_{i}(h)-a_{i}(h+2)=0$, assim $a_{i}(h)$ é constante e $Z(g)=K[Q]$.

No caso $g=S l_{2}(K)$, o módulo de Verma de peso $\lambda \in \mathbb{C}$ é definido por um vetor $v$, de peso máximo $\lambda$ tal que

$$
\begin{array}{ll}
f v=v_{1}, & f v_{i}=v_{i+1}, \\
e v=0, \quad & e v_{i}=i(\lambda-i+1) v_{i-1}, \quad i \geq 1, \\
& h v_{i}=(\lambda-2 i) v_{i}
\end{array}
$$

Proposição 1. O elemento de Casimir $Q$ age sobre $M(\lambda)$ de forma constante, a saber $c=(\lambda+1)^{2}$.

Teorema 14. 1. O módulo $M(\lambda)$ é irredutivel, se e somente se, $\lambda \notin \mathbb{N}-\{0\}$.

2. Para $\lambda=n \in \mathbb{N}-\{0\}$ o módulo $M(\lambda)$ é indecomponível. O módulo $M(-n-2)$ é o único submódulo irredutivel de $M(n)$ e temos $M(n) / M(-n-2) \cong V^{n+1}$, onde $V^{n+1}$ é o módulo de dimensão $n+1$ irredutivel.

Demonstração. 1. Pela teoria dos módulos de Verma temos $\lambda-i+1=0$, se e somente se, $\lambda=i-1$ para $i \in \mathbb{N}_{0}, i \geq 1$. Portanto, $M(\lambda)$ é irredutível se e somente se, $\lambda \notin \mathbb{N}_{0}$.

2. Se $\lambda=n \geq 1$ então $e v_{n+1}=0$ e $h v_{n+1}=(n-2 n-2) v_{n}$. Assim o módulo gerado por $v_{n}, M(-n-2)$ é o único submódulo irredutível de $M(n)$ e além disso $M(n) / M(-n-2) \simeq V^{n+1}$.

Para um $g$-módulo $M$, definimos o anulador de um $g$-módulo $M$ como

$$
A n n_{g}(M)=\{a \in g \mid a m=0 \forall m \in M\} .
$$

\subsubsection{Ideais primitivos de $S l_{2}(K)$}

Definição 12. Seja I um ideal de g. Dizemos que $I$ é um ideal primitivo de $g$, se $I=$ Ann $(M)$, para algum g-módulo irredutivel $M$.

Teorema 15. Seja $\lambda \in \mathbb{C}$. O anulador $A n n_{U(g)}(M(\lambda))$ do módulo de Verma $M(\lambda)$ em $U(g)$ é um ideal bilateral de $U(g)$, gerado pelo elemento $c-(\lambda+1)^{2}$. 
Demonstração. Ver (Mazorchuk, 2009).

Teorema 16. Seja $M$ um $U(g)$-módulo irredutivel.

1. O elemento de Casimir $Q$, age como escalar no módulo $M$.

2. Existe $\lambda \in \mathbb{C}$ tal que $I_{\lambda} \subset A n n_{U(g)}(M)$, onde $I_{\lambda}$ é o ideal primitivo associado a $\lambda$.

Demonstração. 1. Lema de Schur.

2. Se $\mu$ é a ação do elemento de Casimir sobre $M$, e $\lambda$ é raiz do polinômio $(x+1)^{2}-\mu$, então $Q-(\lambda+1)^{2}$ gera o módulo $I_{\lambda} \subset A n n_{U(g)}(M)$.

Para a álgebra nilpotente $\mathfrak{h}$ de dimensão 3 , gerada por $x, y, z \operatorname{com}[x, z]=[y, z]=0$ e $[x, y]=z$, temos o ideal primitivo $I=U(\mathfrak{h})(z-\lambda)$, de $U(\mathfrak{h})$. Da seção anterior, $U / I$ é isomorfo a $A_{1}$.

Seja $K$ um corpo algebricamente fechado de característica zero. Definimos os quocientes primitivos da álgebra envelopante $U_{s}=U\left(S l_{2}\right)$ de $S l_{2}(K)$, por $B_{\lambda}=U s / U_{s}(Q-\lambda)$ como em (Jacques, 1973), onde $I_{\lambda}=U_{s}(Q-\lambda)$ é um ideal primitivo. É claro que $I_{\lambda}=U_{s}(Q-\lambda)$ é um ideal bilateral de $U_{s}$. Definimos também $\operatorname{Primf}\left(U_{s}\right)$ como o conjunto dos ideais primitivos de $U_{s}$, assim temos a aplicação bijetora

$$
K \longrightarrow \operatorname{Primf}\left(U_{s}\right)
$$

dada por $\lambda \longrightarrow I_{\lambda}$. Dixmier prova em (Jacques , 1973), que $I_{\lambda}$ é maximal se $\lambda$ não é da forma $n^{2}+2 n$. Nos outros casos $I_{\lambda} \subset I_{\lambda}^{\prime}$ onde $I_{\lambda} \neq I_{\lambda}^{\prime}$ e $I_{\lambda}^{\prime}$ é de codimensão $(n+1)^{2}$. Os ideais $I_{\lambda}^{\prime}$ são os ideais primitivos de $U_{s}$ de codimensão finita. Note que $B_{\lambda}$ é Noetheriano à direita, à esquerda e seu centro é $K$. Para cada $\alpha \in K$ temos $B_{\lambda}^{\alpha}$, onde $B_{\lambda}^{\alpha}=\left\{b \in B_{\lambda} \mid h b=\alpha b\right\}$. A álgebra $\bigoplus B_{\lambda}^{\alpha}$ é uma graduação de $B_{\lambda}$ isomorfa a $K[X, Y, Z] /\left(4 X Y+Y^{2}\right)$.

Lema 7. $B_{\lambda} \cong B_{\mu}$, se e somente se, $\lambda=\mu$.

Em (Block , 1981), é feita a classificação para uma parte das representações irredutíveis de $S l_{2}(K)$. Estas representações são divididas em representações de $S$-torção e representações livres de $S$-torção. Ilustraremos o mostrado por R. Block em (Block , 1981).

A ação do elemento do Casimir $Q=4 f e+h^{2}+2 h$ sobre o módulo $M(\lambda-1)$ é dada por $\gamma=\lambda^{2}-1$, assim temos o isomorfismo $M(\lambda-1) \cong U_{s} /\left(U_{s}(Q-\gamma)\right)$.

Por outra parte $K[\xi]$, pode ser visto como um $U_{s}$-módulo com a ação determinada por $p=\frac{d}{d \xi}$ e $q$ como a multiplicação por $\xi$. Definimos a aplicação bijetora entre os módulos $K[\xi]$ e $M(\lambda-1)$ como segue

$$
\sum a_{i} \xi^{i} \longrightarrow \sum f^{i} \otimes a_{i}
$$

Como $h\left(f^{i} \otimes 1\right)=(\lambda-2 i-1) f^{i} \otimes 1$ existe uma representação $\varphi$ de $U_{s}$ que faz possível identificar $h$ com o elemento

$$
h \equiv-2 q p+\lambda-1 .
$$


De forma análoga, identificamos $f \equiv q$ e $e \equiv-(q p-\lambda+1) p$. Tomando $\tau \in A$ ut $\left(U_{s}\right)$ como o automorfismo de álgebras que troca $e$ com $f$ e $\tau(h)=-h$ podemos definir o homomorfismo $\rho_{-\lambda}=\rho_{\lambda} \tau$.

A álgebra $U_{s}$ pode-se ver como uma subálgebra da primeira álgebra de Weyl $A_{1}$ através do homomorfismo $\rho_{\lambda}$, dado por

$$
\rho_{\lambda}(e)=q, \quad \rho_{\lambda}(f)=-(q p+\lambda+1) p, \quad \rho_{\lambda}(h)=2 q p+\lambda+1 .
$$

Da definição de $\rho_{\lambda}$ temos

$$
\begin{aligned}
\rho_{\lambda}\left(4 f e+h^{2}+2 h\right) & =-4(q p+\lambda+1) p q+(2 q p+\lambda+1)^{2}+2(2 q p+\lambda+1), \\
& =-4(q p+\lambda+1) q p-4(q p+\lambda+1)+(2 q p+\lambda+1)^{2}+2(2 q p+\lambda+1), \\
& =\lambda^{2}-1
\end{aligned}
$$

Assim vemos que $\rho_{\lambda}$ tem por núcleo o ideal $U_{s}\left(Q-\lambda^{2}+1\right)$ denotado por $\mathcal{B}_{\gamma}$, onde $\gamma=\lambda^{2}-1$.

Considere a família de homomorfismos, denotada por $\sigma_{\gamma}$ e definida como

$$
\sigma_{\gamma}(e)=q, \quad \sigma_{\gamma}(h)=2 q p, \quad \sigma_{\gamma}(f)=\left(\frac{1}{4} \gamma q^{-1}-q p^{2}\right)
$$

Cada um dos homomorfismos anteriores pode ser estendido a álgebra envelopante $U_{s}$. Esta extensão é denotada da mesma forma. Para o caso $\gamma=\lambda^{2}-1$ temos o isomorfismo entre as álgebras $\sigma_{\gamma} U_{s}=U_{s_{\gamma}}$ e $\rho_{\lambda} U_{s}$. Pela definição de $\rho_{\lambda}$ temos o homomorfismo induzido de $\rho_{\lambda} U_{s}$ em $\mathfrak{B}$, além disso $K[q, p] \subset \rho_{\lambda} U_{s}$. Portanto, $S=K[q]-\{0\} \subset \rho_{\lambda} U_{s}$ e $\mathfrak{B}$ é a localização de $\rho_{\lambda} U_{s}$ por $S$.

Lema 8. Suponha $\lambda \in K$ e $\gamma=\lambda^{2}-1$. Então a aplicação $\rho_{\lambda} \rho_{-\lambda}^{-1}: \rho_{\lambda} U_{s} \longrightarrow \rho_{-\lambda} U_{s}$ pode-se estender para um único homomorfismo de $\mathfrak{B}$ que fixa $q$ e envia $p$ para $p-\lambda q^{-1}$.

Para um conjunto de representações irredutíveis de $S l_{2}(K)$, existe uma relação entre os elemento irredutíveis de $\mathfrak{B}$ e os $U_{s}$-módulos irredutíveis, descrita no seguinte lema, demonstrado em (Block , 1981). Além disso estabelece quando um elemento pertence ao $\operatorname{Soc}_{\rho_{\lambda} U_{s}}(N)$, para $N$ um $\mathfrak{B}$-módulo irredutível.

Lema 9. Suponhamos que $u \in U s, \gamma \in K, \lambda \in \bar{K}$ e $\lambda^{2}=\gamma+1$. Seja $G$ o conjunto dos polinômios irredutiveis em $K[q]$. As seguintes afirmações são equivalentes.

$\rho_{\lambda} u, \rho_{-\lambda} u$ são preservados em $q$, e $\sigma_{\gamma} u$ preservado para cada $g \in G-q$.

$\rho_{\lambda} u$ é preservados, e $\rho_{\lambda} u$ é preservados em $q$.

$\rho_{\lambda} u, \rho_{-\lambda} u$ são preservados.

Para $a \in \mathfrak{B}$ as seguintes afirmações são equivalentes

$$
\begin{gathered}
{\overline{\rho_{\lambda}}}_{\overline{\sigma_{\gamma}}}{ }^{-1} a, \rho_{-\lambda}^{-}{\overline{\sigma_{\gamma}}}^{-1} a, \text { são preservados em } q \text {, e } \sigma_{\gamma} \text { u preservado para cada } g \in G-q . \\
a \text { é preservados, e } \overline{\rho_{\lambda}}-\bar{\rho}_{\lambda}{ }^{-1} a \text { é preservados em } q . \\
a, \overline{\rho_{\lambda}}-\bar{\rho}_{\lambda}{ }^{-1} a \text { são preservados. }
\end{gathered}
$$




\subsection{Representações irredutíveis da primeira álgebras de Weyl}

Lema 10. $\mathfrak{B}$ é um domínio de ideais principais.

Demonstração. Sejam $J \triangleleft \mathfrak{B}$ um ideal á esquerda e $b \in J$ de grau mínimo em $p$. Usando o algoritmo da divisão para $a \in J$ existem $x<y \in \mathfrak{B}$ tais que $a=x b+y$ onde grau $(b)>$ $\operatorname{grau}(y)$. Como $y \in J$ então $y=0$. Ver (Ore, 1933).

Suponhamos que $N$ é um $\mathfrak{B}$ módulo irredutível com representação irredutível $\rho$ tal que $\mathfrak{B} /(\operatorname{ker}(\rho)) \simeq N$, assim $N \simeq \mathfrak{B} / \mathfrak{B} F$, onde $\operatorname{ker}(\rho)=\mathfrak{B} F$. O fato que $\mathfrak{B} / \mathfrak{B} F$ é irredutível, implica que $F$ é um elemento de grau mínimo e irredutível em $\mathfrak{B}$.

Lema 11. Sejam $N_{1} \simeq \mathfrak{B} / \mathfrak{B} F, N_{2} \simeq \mathfrak{B} / \mathfrak{B} G$ dois $\mathfrak{B}$-módulo irredutiveis, com $F, G \in \mathfrak{B}$. $N_{1} \simeq N_{2}$, se e somente se, $G$ e $F$ são similares.

Demonstração. Sejam $F, G \in \mathfrak{B}$ tais que $\mathfrak{B} / \mathfrak{B} F \simeq \mathfrak{B} / \mathfrak{B} G$ e $\varphi$ o isomorfismo que existe entre os $\mathfrak{B}$-módulos $\mathfrak{B} / \mathfrak{B} G, \mathfrak{B} / \mathfrak{B} F$. É claro que o isomorfismo $\varphi$ está definido pela imagem de $1+\mathfrak{B} F$, assim $\varphi(1+\mathfrak{B} F)=u+\mathfrak{B} G$ e $0=G \varphi(1+\mathfrak{B} F)$ em consequência $F u=v G$. De forma análoga existem $x, y \in \mathfrak{B}$ tais que $x F=G y$. Mais ainda se $F$ é irredutível, $F$ e $v$ são relativamente primos a esquerda e $G, y$ são relativamente primos a direita.

Se $F, G \in \mathfrak{B}$ são irredutíveis e similares, existem $v, u \in \mathfrak{B}$ tais que $F u=v G$. Seja $\phi$ : $\mathfrak{B} \longrightarrow \mathfrak{B} / \mathfrak{B} G$ o homomorfismo definido por $\phi(f)=f u+\mathfrak{B} G$. É claro que $\mathfrak{B} F \subset \operatorname{ker}(\phi)$, como $\mathfrak{B} F$ é um ideal maximal e $\phi$ não é trivial, temos $\mathfrak{B} F=\operatorname{ker}(\phi)$.

Definição 13. Seja $N$ um $\mathfrak{B}$-módulo irredutível, definimos

$$
\operatorname{Mnn} \operatorname{Ann}(N)=\{F \in \mathfrak{B} \mid F \text { é irredutivel } e \exists x \in N-\{0\}: F x=0\} .
$$

A classe dos $\mathfrak{B}$-módulos irredutíveis isomorfos está em correspondência bijetora com a classe dos polinômios $b \in \mathfrak{B}$ irredutíveis que são similares.

Seja $N$ um $\mathfrak{B}$-módulo irredutível é $F \in M n n A n n(N) \operatorname{com} F=\sum_{i=1}^{k} f_{i}(q) p^{i}, f_{i}(q) \in K(q)$ e $x \in N-\{0\}$ tal que $F x=0$. Então $\left\{x, p x, \cdots, p^{k-1} x\right\}$ é um conjunto l.i. sobre $K(q)$ gerador de $N$ como $K(q)$-espaço vetorial. Se $F_{1}, F_{2} \in \operatorname{MnnAnn}(N)$, então existem $n_{1}, n_{2} \in N-\{0\}$ tais que $F_{i} n_{i}=0$, como $N$ é um $K(q)$-espaço vetorial temos $\operatorname{grau}_{p}\left(F_{1}\right)=\operatorname{grau}_{p}\left(F_{2}\right)$.

Definição 14. Dizemos que um $\mathfrak{B}$ módulo irredutivel $N$ é de grau $k$, se existe $F \in \operatorname{MnnAnn}(N)$ tal que $k=\operatorname{grau}_{p} F$. Do anterior $k$ é independente da escolha de $F$.

Sejam $M$ um $\mathfrak{B}$-módulo irredutível não nulo e $F \in A_{1} \cap A n n_{\mathfrak{B}}(M)$ irredutível e preservado, de (Block, 1981), $A_{1} \cap \mathfrak{B} F$ é um ideal maximal de $A_{1}, \log o=\left(A_{1}+\mathfrak{B} F\right) / \mathfrak{B} F$ é um $A_{1}$-módulo irredutível, livre de $S$-torção e $S^{-1} N=M$. Além disso o $S_{o c} c_{A_{1}}(M)$ resulta irredutível e $S_{o c} c_{A_{1}}(M) \simeq N$. Se $F s^{-1}$ é preservado para $s \in S$, e $F m=0$ para algum $m \in M$, 
então $s m \in S o c_{A_{1}}(M)$.

No conjunto dos $A_{1}$-módulos irredutíveis, definimos a relação de equivalência $\sim$, onde $N_{1} \sim$ $N_{2}$, se e somente se, $N_{1}$ e $N_{2}$ são isomorfos. Denotares por $A_{1}^{\Delta}(i r r)$ ao conjunto das classes de equivalência geradas pela relação de equivalência $\sim$. Definimos a mesma relação de equivalência no conjunto dos $\mathfrak{B}$-módulos irredutíveis, e denotamos por $\mathfrak{B}^{\Delta}($ irr $)$ ao conjunto das classes de equivalência geradas por esta relação de equivalência. Por outro lado, no conjunto dos polinômios irredutíveis de $\mathfrak{B}$ definimos a relação de equivalência $\sim$, onde $F_{1} \sim F_{2}$ para $F_{1}, F_{2} \in \mathfrak{B}$, se e somente se, $F_{1}, F_{2}$ são similares, denotamos $\mathfrak{B}^{\triangle}(\operatorname{Irr}(\mathfrak{B}))$ o conjunto das clases de equivalência gerado por $\sim$. Assim temos uma bijeção entre os conjuntos $\mathfrak{B}^{\triangle}($ irr $)$ e $\mathfrak{B}^{\triangle}(\operatorname{Irr}(\mathfrak{B}))$.

Para cada $A_{1}$-módulo irredutível livre de $S$-torção $M$, temos o $\mathfrak{B}$-módulo irredutível $S^{-1} M$, onde $S=K[q]-\{0\}$.

Sejam $N$ um $\mathfrak{B}$-módulo irredutível e $\operatorname{irr}_{A_{1}}(N)$ o conjunto dos $A_{1}$-submódulo irredutíveis de $N$ livres de $S$-torção, assim $S_{o c} A_{1}(N)=\sum_{M \in I r r_{A_{1}}(N)} M$. Como $S^{-} M_{1}=S^{-} M_{2}$ para $M_{1}, M_{2} \in \operatorname{Irr}_{A_{1}}(N)$, então $M_{1} \cap M_{2} \neq \varnothing$, portanto $\operatorname{Soc}_{A_{1}}(N)$ é um $A_{1}$-módulo irredutível.

Teorema 17. Existe uma bijeção entre a classe dos $A_{1}$-módulos irredutíveis e os $\mathfrak{B}$-módulos irredutiveis.

Demonstração. Suponhamos que $S^{-1} M_{1} \cong S^{-1} M_{2}$, onde $M_{1}, M_{2}$ são $A_{1}$-submódulos irredutíveis. Então $M_{1} \simeq M_{2}$ como $A_{1}$-módulos e consequentemente $S^{-1}$ é injetor. Por outro lado temos o isomorfismo entre $S^{-1} S_{o c_{A_{1}}}(N)$ e $N$, para $N$ um $\mathfrak{B}$-módulo simples, obtido pela extensão do homomorfismo inclusão de $\operatorname{Soc}_{A_{1}}(N) \rightarrow N$ e pela propriedade universal.

Seja $I$ um ideal primo de $K[q]$. Para $f \in K[q]$ definimos $v_{I}(f)=r$, onde $r$ é o inteiro tal que $f \in I^{r}-I^{r-1}$. A extensão da avaliação $v_{I}$ ao corpo $T=S^{-1} K[q]$, onde $S=K[q]-\{0\}$ é denotada por $\nu_{I}$. Onde $\nu_{I}\left(\frac{f}{g}\right)=v_{I}(f)-v_{I}(g)$ para $f, g \in K[q]$. A avaliação $\nu_{I}$ pode-se estender para uma avaliação de $\mathfrak{B}$, denotada também por $\nu_{I}$ e definida para cada $F=$ $\sum_{i-0}^{n} b_{i} p^{i} \in \mathfrak{B}$ por

$$
v_{I}(F)=\min \left\{v_{I}\left(b_{i}\right)-i \mid i \geq 0\right\} .
$$

Definição 15. Seja $F=\sum_{i=0}^{k} b_{i} p^{i} \in \mathfrak{B}$ onde $b_{i} \in K(q)$. Dizemos que $\alpha$ é um polo de $F$, se $\alpha$ é polo de algum quociente $b_{j} / b_{k}$. Denotamos o conjunto dos polos de $F \operatorname{por} \operatorname{Pol}(F)$.

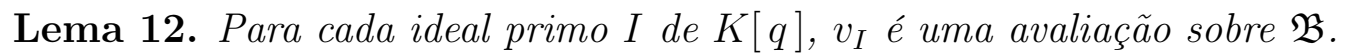

Demonstração. Ver demonstração em (Block, 1981).

Para o ideal primo $P=K[q](q-\alpha)$ de $K[q]$ e $F \in \mathfrak{B}$, definimos o polinômios $Q_{\alpha, F}(\xi)$, que chamamos de polinômios principal de $F$, para $F=\sum b_{i} p^{i} \in B$, como

$$
Q_{\alpha, F}(\xi)=\sum \eta_{P}\left\{g^{-v_{P}(b)-j} b_{j}(\partial g)^{j}\right\} \xi(\xi-1) \cdots(\xi-(j-1)) .
$$


Onde $\eta_{P}$ é o homomorfismo canônico de $K[q]_{P}$ em $K_{P}=K[q]_{P} / P K[q]_{p} \cong K[q] / P$. Para $g=q-\alpha$ temos $v_{P}\left(b_{j}(\partial g)^{j}\right)=v_{P}\left(v_{j}\right)+j v_{P}(\partial g)$. Como $\partial g \notin P$ então $v_{P}(\partial g)=0$, segue-se $v_{P}\left(b_{j}(\partial g)^{j}\right)=v_{P}\left(b_{j}\right) \geq v_{P}(b)+j$. Portanto, $\eta_{P}\left\{g^{-v_{P}(F)-j} b_{j}(\partial g)^{j}\right\}$ está bem definida.

Definição 16. Dizemos que $F \in \mathfrak{B}$ não nulo é preservado respeito a $\alpha$, se o polinômio $Q_{\alpha, F}(\xi)$ não possui raízes inteiras negativas.

O polinômio $F \in \mathfrak{B}$ é preservado se ele é preservado respeito a cada um de seus polos. Para o polinômio principal temos as seguintes propriedades, ver (Block, 1981).

Lema 13. Suponhamos que $G, F \in \mathfrak{B}$ e $\alpha \in K$ então $Q_{\alpha, G F}(\xi)=Q_{\alpha, G}\left(\xi+\nu_{\alpha}(F)\right) Q_{\alpha, F}(\xi)$.

Seja $F=\sum b_{i} p^{i} \in \mathfrak{B}$ de grau $k>0$. Definimos o especial primo de $\mathfrak{B}$, como o ideal primo $P$ de $K[q]$, tal que $v_{P}(F)=v_{P}\left(b_{i}\right)-i$ para algum $i<k$. Notemos que os primos especiais de $F$ são $K[q](q-\alpha)$ onde $\alpha$ é um polo de $F$. Se $\alpha$ não é polo de $F$, então $v_{P}\left(b_{k}\right)<v_{P}\left(b_{i}\right)$ para todo $i<k$. Assim $v_{P}(F)=v_{P}\left(b_{k}\right)-k$ e portanto os primos especiais de $F$ são finitos e estão caracterizados pelos $\alpha$ tais que $v_{P}\left(b_{k}\right)>v_{P}\left(b_{i}\right)$ para algum $i<k$, isto é pelos polos de $F$. Doravante denotaremos a avaliação $v_{P}$ por $\nu_{\alpha}$.

Definição 17. Sejam $F \in \mathfrak{B}$ e $\alpha \in \operatorname{Pol}(F)$, então as raízes do polinômio $Q_{\alpha, F}(\xi)$ são chamadas raizes principais de $F$ relativas a $\alpha$.

Lema 14. Sejam $0 \neq G_{1}, \cdots, G_{j} \in \mathfrak{B}$, então existe $s \in K[q]$ tal que $G_{i} s^{-1}$ é preservado para todo $i=1, \cdots, j$. $\operatorname{Ver}$ (Block, 1981).

Teorema 18. Seja $F=\sum b_{i} p^{i} \in \mathfrak{B}$ irredutivel e preservado. Então o $A_{1}$-módulo $A_{1} /\left(A_{1} \cap\right.$ $\mathfrak{B} F)$ é simples.

Teorema 19. Todo $A_{1}$-módulo irredutivel de $S$-torção $M$ é isomorfo a $K[p]$, onde $p$ age pela multiplicação e q como $\frac{d}{d p}-\alpha$, para algum $\alpha \in K$. Este módulo será denotado por $M(\alpha)$.

Demonstração. Seja $M$ um $A_{1}$-módulo irredutível de $S$-torção não nulo. Então existe $m \in M$ não nulo tal que $(q-\alpha) m=0$, para algum $\alpha \in K$. Se $p^{k} m=p^{t} m$ para $k<t$, então $(q-\alpha)^{t} p^{k} m=(q-\alpha)^{t} p^{t} m=0$. Logo $m=0$ e $M=0$. Portanto, $p^{k} m \neq p^{t} m$ para $k \neq t$ e em consequência $M$ é isomorfo a $M(\alpha)$.

Lema 15. Seja $L$ é um ideal maximal de $\mathfrak{B}$. Então $A_{1} \cap L$ é um ideal maximal de $A_{1}$, se e somente se, para todo $A_{1}$-módulo não nulo de $S$-torção irredutivel $M$, existe um elemento em $A_{1} \cap L$ que age de forma injetiva sobre o módulo $M$.

Demonstração. Ver (Block, 1981), Teorema 4.3.

Suponhamos que $L^{\prime}$ age injetivamente sobre $M$. Suponha $L^{\prime} \subset J$ um ideal máximal de $A_{1}$. Se $A_{1} / J$ é livre de s-torção, então $S^{-1} J$ é um ideal máximal a esquerda de $\mathfrak{B}, J=S^{-1} A_{1} \cap J \supset$ $L^{\prime}$, dado que $L$ é maximal deve-se ter $L=J$. Se $A_{1} / J$ é de $S$-torção, então existe $a \in L^{\prime}$ tal que $a \bar{f} \neq 0$ para todo $\bar{f} \in A_{1} / J$, isto é absurdo pois $L^{\prime} \subset J$. 
Lema 16. Se $(q-\alpha)^{-v_{\alpha}(F)} F$ age de forma injeta sobre $M(\alpha)$, então $F \in \mathfrak{B}$ é preservado em $\alpha$.

Lema 17. Seja $N$ um $\mathfrak{B}$-módulo irredutível. Então $\operatorname{Soc}_{A_{1}}(N) \neq 0$. Para todo $A_{1}$-módulo irredutivel e livre de $S$-torção $M$, existe $F \in B$ irredutivel e preservado tal que $M \simeq$ $A_{1} /\left(A_{1} \cap \mathfrak{B} F\right)$.

Sejam $N$ um $\mathfrak{B}$-módulo irredutível e $F \in \mathfrak{B}$ irredutível e preservado tal que $F n=0$, para algum $0 \neq n \in N$. Do isomorfismo $N \cong \mathfrak{B} / \mathfrak{B} F$ o módulo $N^{\prime}=\left(A_{1} s+\mathfrak{B} F\right) / \mathfrak{B} F$ é um $A_{1}$-submódulo de $N$, onde $N^{\prime} \cong A_{1} s /\left(A_{1} s \cap \mathfrak{B} F\right) \cong A_{1} /\left(A_{1} \cap \mathfrak{B} F s^{-1}\right)$, assim $N^{\prime}$ é irredutível, se e somente se, $F s^{-1}$ é preservado. Do anterior e usando o fato que $S_{o c_{A_{1}}}(N)$ é irredutível segue-se que $s n \in \operatorname{Soc}_{A_{1}}(N)=A s n$. Do anterior, para cada polo $\alpha$ de $F$ tal que $Q_{\alpha, F}(\xi)$ possui uma raiz inteira, existe um $t \in \mathbb{Z}$ tal que $(q-\alpha)^{-t} n \notin \operatorname{Soc}_{A_{1}}(N)$.

O seguinte teorema demonstrado por Block em (Block, 1981), estabelece condições necessárias e suficientes para decidir quando um elemento estão no $\operatorname{Soc}_{A_{1}}(N)$.

Teorema 20. Seja $N$ um $\mathfrak{B}$-modulo irredutivel, então $\operatorname{Soc}_{A_{1}} N \neq 0$.

Exemplo 2. Para $p-t \in \mathfrak{B}$ irredutível, de (Block , 1981) temos

$$
\operatorname{Soc}_{A_{1}}(N)=K\left[q,\left(q-\alpha_{1}\right)^{-1}, \cdots,\left(q-\alpha_{l}\right)^{-1}\right]
$$

onde $N \simeq \mathfrak{B} /\left(\mathfrak{B}\left(p-t_{1}\right)\right), l$ é o número de polos de $t_{1}$ e $p-t_{1}$ é similar a $p-t$.

De (Ore , 1933), dois polinômios $p-t_{1}$ e $p-t_{2}$ são similares, se e somente se, $t_{1}-t_{2}=\frac{c^{\prime}}{c}$ para $c \in K(q)$. Logo $p-t$ e $p-t-\frac{j}{q-\alpha}$ são similares, assim $p-t$ e $p-t-\sum_{\alpha} \operatorname{Res}_{\alpha}(t)(q-\alpha)^{-1}$ sempre que $\operatorname{Res}_{\alpha}(t) \in \mathbb{Z}$. Portanto existe $d=p-t_{1}$ similar a $p-t$, onde $\operatorname{Res}_{\alpha}\left(t_{1}\right) \notin \mathbb{Z}$, para cada polo $\alpha$ de $d$. Damos estrutura a $K(q)$ de $\mathfrak{B}$-módulo irredutível baixo a ação de $p$ definida como; $p . f=f^{\prime}+t_{1} f$.

Seja $\left\{\alpha_{1}, \cdots, \alpha_{l}\right\}$ o conjunto dos polos de $d$ e $W=\operatorname{Soc}_{A_{1}}(N)$. Se $m_{j}$ é o maior inteiro tal que $\left(q-\alpha_{j}\right)^{-m_{j}} \in W$ temos

$$
p\left(q-\alpha_{j}\right)^{-m_{j}}=t_{1}\left(q-\alpha_{j}\right)^{-m_{j}}+m_{j}\left(q-\alpha_{j}\right)^{-m_{j}-1},
$$

então $\left(\operatorname{Res}_{\alpha_{j}}\left(t_{1}\right)+m_{j}\right)\left(q-\alpha_{j}\right)^{-m_{j}-1} \in W$. Como $\operatorname{Res}_{\alpha_{i}}\left(t_{1}\right)+m_{i} \neq 0$ temos $\left(q-\alpha_{i}\right)^{-m_{i}-1} \in W$. Note que $\left(q-\alpha_{j}\right)^{-n} \in W$ para todo $n \in \mathbb{N}$, sempre que $v_{\alpha_{j}}(t)<-1$. 


\section{Capítulo 2}

\section{Módulos de grau dois sobre a primeira álgebra de Weyl}

Seja $N$ um $\mathfrak{B}$-módulo irredutível de grau dois. Para $x \in N-\{0\}$ existe um polonòmio $F=p^{2}+c_{1} p+c_{0} \in \mathfrak{B}$ irredutível tal que $F x=0$, e portanto $N \simeq \mathfrak{B} / \mathfrak{B} F$. Para o polinômio $F$, definimos $\Delta(F)=\left\{\alpha \in \operatorname{Pol}(F) \mid \exists n \in \mathbb{Z}: Q_{\alpha, F}(n)=0\right\}$ e $-n_{\alpha}$ como a menor raiz inteira negativa. Pelo trabalho de R. Block (Block, 1981), existe um polinômio $G_{1}=p^{2}+a_{1} p+a_{0} \in \mathfrak{B}$ irredutível, preservado e similar a $F$ tal que $N \simeq \mathfrak{B} / \mathfrak{B} G_{1}$ e $\Delta\left(G_{1}\right)=\left\{\alpha \in \operatorname{Pol}\left(G_{1}\right) \mid Q_{\alpha, F}(0)=0\right\}$. Damos uma relação entre o módulo $A_{1}\left(\begin{array}{l}1 \\ 0\end{array}\right)$ e o polinômio $F_{1}=p^{2}+a p+b$. O polinômio $F_{1}$ é um polinômio similar a $F$ preservado e com umas características que nós permitem dar uma melhor descrição de $W=\operatorname{Soc}_{A_{1}}(N)$. Além disso Determinaremos a relação que existe entre os $A_{1}$-módulos $W$ e $K_{P o l\left(F_{1}\right)} \times K_{P o l\left(F_{1}\right)}$, onde $K_{\text {Pol }\left(F_{1}\right)}=K\left[q,\left(q-\alpha_{1}\right)^{-1}, \cdots,\left(q-\alpha_{m}\right)^{-1}\right]$ e $\operatorname{Pol}(F)=\left\{\alpha_{1}, \cdots, \alpha_{m}\right\}$, usando o polinômio $F_{1}$ e o módulo quociente destes módulos. Os principais resultados destas seções são:

Teorema 21. Seja $F \in M n n A n n(N)$, então o módulo $W=S_{o c} A_{A_{1}}(N)$ é irredutivel e da seguinte forma

1. $W=A_{1}\left(\begin{array}{l}1 \\ 0\end{array}\right)$ se, e somente se, F é preservado.

2. $W=A_{1}\left(\begin{array}{c}\prod_{\alpha \in \Delta(F)}(q-\alpha)^{n_{\alpha}} \\ 0\end{array}\right)$ se, e somente se, $Q_{\alpha, F}\left(-n_{\alpha}\right)=0$ para $0<n \in \mathbb{N} e$ $\alpha \in \Delta(F)$.

Teorema 22. $W=K_{P o l(F)} \times K_{P o l(F)}$ se, e somente se, $\Delta\left(F_{1}\right)=\emptyset$.

Para cada $\alpha \in \operatorname{Pol}\left(F_{1}\right)$ existe $h_{\alpha} \in K[q]$ de grau mínimo tal que os polinômios $h_{\alpha} a, h_{\alpha} b \in$ $K(q)$ só tem polo em $\alpha$. O conjunto $T(\alpha)=\left\{h_{\alpha}^{n-1} p^{n}\left(\begin{array}{l}1 \\ 0\end{array}\right) \mid 2 \leq n \in \mathbb{N}\right\}$ gera um sub-espaço de $W$. Usando $T(\alpha)$ temos $\Delta_{0}(F)=\left\{\alpha \in \Delta(F) \mid \operatorname{dim}_{K}\left(T(\alpha) \cup\left\{\left(\begin{array}{l}1 \\ 0\end{array}\right), p\left(\begin{array}{l}1 \\ 0\end{array}\right)\right\}\right)<\infty\right\}$.

Teorema 23. Suponhamos que $\Delta\left(F_{1}\right) \neq \emptyset$, então

1. $K_{P o l(F)} \times K_{P o l(F)} / W$ é um $A_{1}$-módulo de $S$-torção isomorfo a $\bigoplus_{\alpha \in \Delta\left(F_{1}\right)} M(\alpha)$, onde $M(\alpha)$ é o $A_{1}$-módulo irredutivel de $S$-torção descrito no capitulo anterior.

2. O $A_{1}$-módulo $K_{P o l(F)} \times K_{P o l(F)}$ é de comprimento finito $\left|\Delta\left(F_{1}\right)\right|+\left|\Delta_{0}\left(F_{1}\right)\right|$ e cíclico. 
Onde o módulo $W$ é módulo $\operatorname{Soc}_{A_{1}}(N)=A_{1}\left(\begin{array}{l}1 \\ 0\end{array}\right)$.

\subsection{Propriedades do polinômio $G_{1}$}

A continuação daremos uma construção do polinômio $G_{1}$. Seja $F=p^{2}+a_{1} p+a_{2} \in$ $M n n \operatorname{Ann}(N)$ e $\alpha \in \operatorname{Pol}(F)$, então do capítulo anterior temos

$$
v_{\alpha}(F)=\min \left\{-2, \nu_{\alpha}\left(a_{1}\right)-1, \nu_{\alpha}\left(a_{2}\right)\right\} .
$$

Como o polinômio principal associado a $F$ em $\alpha \in \operatorname{Pol}\left(F_{1}\right)$ é dado por

$$
Q_{\alpha, F}(\xi)=\eta_{\alpha}\left((q-\alpha)^{-v_{\alpha}(F)-j}\right) \xi(\xi-1)+\eta_{\alpha}\left((q-\alpha)^{-v_{\alpha}(F)-j} a_{1}\right) \xi+\eta_{\alpha}\left((q-\alpha)^{-v_{\alpha}(F)-j} a_{2}\right),
$$

então o polinômio $Q_{\alpha, F}(\xi)$ tem uma das seguintes formas:

$$
Q_{\alpha, F}(\xi)= \begin{cases}(\xi-1) \xi & \nu_{\alpha}\left(a_{1}\right) \geq 0, \nu_{\alpha}\left(a_{2}\right)=-1 . \\ (\xi-1) \xi+\operatorname{Res}_{\alpha}\left(a_{2}\right) & \nu_{\alpha}\left(a_{1}\right) \geq 0, \nu_{\alpha}\left(a_{2}\right)=-2 . \\ \xi\left(\xi-1+\operatorname{Res}_{\alpha}\left(a_{1}\right)\right) & \nu_{\alpha}\left(a_{1}\right)=-1, \nu_{\alpha}\left(a_{2}\right) \geq-1 . \\ (\xi-1) \xi+\operatorname{Res}_{\alpha}\left(a_{1}\right) \xi+\operatorname{Res}_{\alpha}\left(a_{2}\right) & \nu_{\alpha}\left(a_{1}\right)=-1, \nu_{\alpha}\left(a_{2}\right)=-2 . \\ \operatorname{Res}_{\alpha}\left(a_{1}\right) \xi & \nu_{\alpha}\left(a_{1}\right) \leq-2, \nu_{\alpha}\left(a_{2}\right) \geq \nu_{\alpha}\left(a_{1}\right) . \\ \operatorname{Res}_{\alpha}\left(a_{1}\right) \xi+\operatorname{Res}_{\alpha}\left(a_{2}\right) & \nu_{\alpha}\left(a_{1}\right) \leq-2, \nu_{\alpha}\left(a_{2}\right)=-1+\nu_{\alpha}\left(a_{1}\right) . \\ \operatorname{Res}_{\alpha}\left(a_{2}\right) & \nu_{\alpha}\left(a_{2}\right) \leq-3, \nu_{\alpha}\left(a_{2}\right)<\nu_{\alpha}\left(a_{1}\right)-1 .\end{cases}
$$

Para cada $\alpha \in \operatorname{Pol}(F)$, os polinômios $H=(q-\alpha)^{-n} F(q-\alpha)^{n}$ e $F$ são similares. Assim para $n \in \mathbb{N}$ segue-se

$$
Q_{\alpha, H}(0)=0 \Leftrightarrow Q_{\alpha, F}(n)=0 .
$$

Definimos o conjunto, $\Delta(F)=\left\{\alpha \in \operatorname{Pol}(F) \mid Q_{\alpha, F}(n)=0\right.$, para algum, $\left.n \in \mathbb{N}\right\}$.

Para cada $\alpha \in \Delta(F)$, definimos $\varrho_{\alpha}(F)$ como a menor raiz inteira de $Q_{\alpha, F}(\xi)$. Então o polinômio

$$
G_{1}=F \prod_{\alpha \in \Delta(F)}(q-\alpha)^{\varrho_{\alpha}(F)},
$$

é preservada, e $\Delta\left(G_{1}\right)=\left\{\alpha \in \operatorname{Pol}\left(G_{1}\right) \mid Q_{\alpha, G_{1}}(0)=0\right\}$. Para $y=\prod_{\alpha \in \Delta(F)}(q-\alpha)^{-\varrho_{\alpha}(F)} x$ temos $G_{1} y=0$. Logo, a classe dos $\mathfrak{B}$-módulos irredutíveis isomorfos a $N$, está em correspondência com a classe dos polinômios irredutíveis de grau dois similares a $G_{1}$.

Definimos $\Delta^{\prime}\left(G_{1}\right)$ como o conjunto dos polos de $G_{1}$ tal que $Q_{\alpha, G_{1}}(0) \neq 0$, então

$$
\begin{aligned}
\Delta^{\prime}\left(G_{1}\right) & =\left\{\alpha \in \operatorname{Pol}\left(G_{1}\right) \mid Q_{\alpha, G_{1}}(n) \neq 0, \forall n \in \mathbb{N}\right\} . \\
\Delta\left(G_{1}\right) & =\left\{\alpha \in \operatorname{Pol}\left(G_{1}\right) \mid Q_{\alpha, G_{1}}(0)=0\right\}
\end{aligned}
$$

e $\operatorname{Pol}\left(G_{1}\right)=\Delta\left(G_{1}\right) \cup \Delta^{\prime}\left(G_{1}\right)$ 
Para $\alpha \in \Delta^{\prime}\left(G_{1}\right)$ temos

$$
Q_{\alpha, G_{1}}(\xi)= \begin{cases}(\xi-1) \xi+\operatorname{Res}_{\alpha}\left(a_{2}\right) & \nu_{\alpha}\left(a_{1}\right) \geq 0, \nu_{\alpha}\left(a_{2}\right)=-2 . \\ (\xi-1) \xi+\operatorname{Res}_{\alpha}\left(a_{1}\right) \xi+\operatorname{Res}_{\alpha}\left(a_{2}\right) & \nu_{\alpha}\left(a_{1}\right)=-1, \nu_{\alpha}\left(a_{2}\right)=-2 . \\ \operatorname{Res}_{\alpha}\left(a_{1}\right) \xi+\operatorname{Res}_{\alpha}\left(a_{2}\right) & \nu_{\alpha}\left(a_{1}\right) \leq-2, \nu_{\alpha}\left(a_{2}\right)=-1+\nu_{\alpha}\left(a_{1}\right) . \\ \operatorname{Res}_{\alpha}\left(a_{2}\right) & \nu_{\alpha}\left(a_{2}\right) \leq-3, \nu_{\alpha}\left(a_{2}\right)<\nu_{\alpha}\left(a_{1}\right)-1 .\end{cases}
$$

Para $\alpha \in \Delta\left(G_{1}\right)$ temos

$$
Q_{\alpha, G_{1}}(\xi)= \begin{cases}(\xi-1) \xi & \nu_{\alpha}\left(a_{1}\right) \geq 0, \nu_{\alpha}\left(a_{2}\right)=-1 \\ \xi\left(\xi-1+\operatorname{Res}_{\alpha}\left(a_{1}\right)\right) & \nu_{\alpha}\left(a_{1}\right)=-1, \nu_{\alpha}\left(a_{2}\right) \geq-1 \\ \operatorname{Res}_{\alpha}\left(a_{1}\right) \xi & \nu_{\alpha}\left(a_{1}\right) \leq-2, \nu_{\alpha}\left(a_{2}\right) \geq \nu_{\alpha}\left(a_{1}\right)\end{cases}
$$

Doravante vamos supor que $G_{1}=p^{2}+a p+b$ é o polinomio anulador para algum $n \in N-\{0\}$, onde $a, b \in K(q)$ são como segue

$$
a=\frac{a_{1}}{\prod\left(q-\alpha_{i}\right)^{r_{i}}}, b=\frac{b_{1}}{\prod\left(q-\alpha_{i}\right)^{s_{i}}} .
$$

A maior raiz inteira do polinômio $Q_{\alpha, G_{1}}(\xi)$ é denotadas por $\theta_{\alpha}\left(G_{1}\right)$.

No desenvolvimento do trabalho é relevante determinar um subconjunto dos polos de $\operatorname{Pol}\left(G_{1}\right)$ que seja mínimo. No seguinte exemplo mostramos dois polinômios que ilustram o anterior.

Exemplo 3. Suponhamos que $\nu_{\alpha}(a)=-1, \nu_{\alpha}(b) \geq 0$ com $\operatorname{Res}_{0}(a) \in \mathbb{Z}^{-}$. Nosso interesse é saber quando podemos ter $a-g=\operatorname{Res}_{0}(a)(q-\alpha)^{-1}$. Suponhamos que $\nu_{\alpha}(g) \geq 0$, fazendo $b=\lambda(q-\alpha)^{-\operatorname{Res}_{\alpha}(a)}$ e $f=g^{\prime}+b$ temos que o polinômio $F_{1}=p^{2}+\left(g+\operatorname{Res}_{\alpha}(a)(q-\alpha)^{-1}\right) p+$ $\lambda(q-\alpha)^{-\operatorname{Res}_{\alpha}(a)}$ é similar ao polinômio $F_{2}=p^{2}+g p+g^{\prime}+\lambda(q-\alpha)^{-\operatorname{Res}_{\alpha}(a)}$.

Definição 18. Dizemos que um polo $\alpha$ de um polinômio $F \in \mathfrak{B}$ é essencial, se $\alpha$ é polo de todo polinômio similar a $F$.

Suponhamos que existem $F_{2}=p^{2}+g p+f \in \mathfrak{B}$ similar ao polinômio $G_{1}$ e $\alpha \in \operatorname{Pol}\left(G_{1}\right)$, tais que $\alpha \notin \operatorname{Pol}\left(F_{2}\right)$ e $\operatorname{Pol}\left(F_{2}\right) \subset \operatorname{Pol}\left(G_{1}\right)$. Como $G_{1}$ e $F_{2}$ são similares, existem polinômios $z=x p+x_{0}, y=x p+x_{1} \in \mathfrak{B}$ tais que

$$
\begin{aligned}
\left(x p+x_{0}\right)\left(p^{2}+a p+b\right) & =\left(p^{2}+g p+f\right)\left(x p+x_{1}\right), \\
\xi\left(\xi-1+\operatorname{Res}_{\alpha}(a)\right) Q_{\alpha, z}(\xi-2) & =\left(\xi+\nu_{\alpha}(y)\right)\left(\xi+\nu_{\alpha}(y)-1\right) Q_{\alpha, y}(\xi) .
\end{aligned}
$$

Se $x \notin K$, da equação (2.6) temos

$$
\begin{aligned}
x_{0}+x(a-g) & =2 x^{\prime}+x_{1} . \\
x^{\prime \prime}+g x^{\prime}+f x+g x_{1}+2 x_{1}^{\prime} & =x b+x_{0} a+x a^{\prime} . \\
x_{1}^{\prime \prime}+g x_{1}^{\prime}+f x_{1} & =x_{0} b+x b^{\prime} .
\end{aligned}
$$


De (2.6), $\nu_{\alpha}(y) \leq 1$. Dado que $x p+x_{0}$ é similar a $x p+x_{1}$, existe $c \in K(q)$ tal que

$$
\frac{x_{0}}{x}-\frac{x_{1}}{x}=\frac{c^{\prime}}{c} .
$$

Lema 18. Seja $\alpha \in \operatorname{Pol}\left(G_{1}\right)$. Se Res $\operatorname{Re}_{\alpha}(a) \notin \mathbb{Z}^{-}$, então $\alpha$ é um polo essencial.

Demonstração. Dado que $z$ e $y$ são similares, existe $c \in K(q)$ tal que

$$
\frac{x_{0}}{x}-\frac{x_{1}}{x}=c^{\prime} c^{-1}
$$

portanto $\operatorname{Res}_{\alpha}(a)=2 \nu_{\alpha}(x)+\nu_{\alpha}(c)$.

Das equações a acima, seguem-se as seguintes observações.

Observação 2. 1. Os polos do conjunto $\Delta^{\prime}\left(G_{1}\right)$ são essenciais.

2. Suponhamos que $\nu_{\alpha}(a) \leq-2, \nu_{\alpha}(b) \geq \nu_{\alpha}(a)$. Então $Q_{\alpha, y}(\xi)$ é constante e $\nu_{\alpha}(y)=0$ ou $\nu_{\alpha}(y)=1$. Se $\nu_{\alpha}(y)=0$ de $(2.6)$ e $(2.6)$, temos $\nu_{\alpha}(x)>1, \nu_{\alpha}\left(x_{1}\right)=0$ e $\nu_{\alpha}\left(x_{0}\right)=$ $\nu_{\alpha}(x)-1, \log \nu_{\alpha}(a)=0$. Se $\nu_{\alpha}\left(x_{1}\right)=1$, temos $\nu_{\alpha}(a)=-1$. Portanto o polo $\alpha$ é essencial.

3. Se $\alpha \in \operatorname{Pol}\left(G_{1}\right)$ não é essencial, então $\nu_{\alpha}(a)=-1, \nu_{\alpha}(b)=-1,0$ e $\operatorname{Res}_{\alpha}(a) \in \mathbb{Z}$.

Afirmação 3. Seja $\alpha \in \operatorname{Pol}\left(G_{1}\right)$ tal que $\nu_{\alpha}(a)=-1$ e $\nu_{\alpha}(b) \geq-\operatorname{Res}_{\alpha}(a)$, então $\alpha$ não é essencial.

Demonstração. O homomorfismo $\varphi: W \longrightarrow K_{P o l\left(G_{1}\right)-\{\alpha\}} \times K_{P o l\left(G_{1}\right)-\{\alpha\}}$ definido por

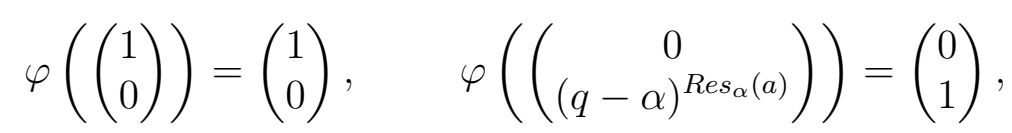

é um homomorfismo injetor de $A_{1}$-módulos. Portanto, o polo $\alpha$ não é essencial.

Afirmação 4. Se $G_{1}=p^{2}+a p+a b$ e similar a $F_{2}=p^{2}+g p+f$, e $\alpha \in \operatorname{Pol}\left(G_{1}\right)$ não é polo de $F_{2}$, então $a-g$ é uma derivação logarítmica.

Demonstração. De (2.8) e usando o fato que $x_{1}-x_{0}=\frac{c^{\prime}}{c}$ temos $a-g=\frac{2 x^{\prime}}{x}+\frac{c^{\prime}}{c}$.

Resta responder; que polos do polinômio $G_{1}$ são essenciais. Responderemos esta pergunta com ajuda da estrutura de $W$.

\subsection{Estrutura de W}

Estudar a estrutura do módulo $N$, equivale a estudar a estrutura de $K(q) \times K(q)$. O nosso objetivo é mostrar mediante a ação de $A_{1}$ em $K(q) \times K(q)$ que

$$
\operatorname{Soc}_{A_{1}}(N) \simeq \operatorname{Soc}_{A_{1}}(K(q) \times K(q))=A_{1}\left(\begin{array}{l}
1 \\
0
\end{array}\right),
$$


usando um método diferente ao método usado por R. Block em (Block, 1981). Adicionalmente daremos uma relação entre as estrutura dos módulos $A_{1}\left(\begin{array}{l}1 \\ 0\end{array}\right)$ e

$$
\left(K_{P o l\left(F_{1}\right)} \times K_{P o l\left(F_{1}\right)}\right) / A_{1}\left(\begin{array}{l}
1 \\
0
\end{array}\right)
$$

A ação da álgebra de Weyl $A_{1}$, sobre o módulo $K(q) \times K(q)$ está definida como segue

$$
p\left(\begin{array}{l}
f \\
g
\end{array}\right)=\left(\begin{array}{cc}
\frac{d}{d q} & -b \\
1 & \frac{d}{d q}-a
\end{array}\right)\left(\begin{array}{l}
f \\
g
\end{array}\right), \quad \text { e } \quad q\left(\begin{array}{l}
f \\
g
\end{array}\right)=\left(\begin{array}{cc}
q & 0 \\
0 & q
\end{array}\right)\left(\begin{array}{l}
f \\
g
\end{array}\right) .
$$

Portanto, o módulo $W$ é um submódulo de $K_{P o l\left(G_{1}\right)} \times K_{P o l\left(G_{1}\right)}$.

\subsubsection{W é irredutivel}

A demonstração da irredutibilidade de $W=A_{1}\left(\begin{array}{l}1 \\ 0\end{array}\right)$ é dividida em três partes. Primeiro mostraremos que todo submódulo de $W$ contem um elemento da forma $\left(\begin{array}{l}f \\ 0\end{array}\right),\left(\begin{array}{l}0 \\ f\end{array}\right)$, para $f \in K[q]$. Em seguida mostraremos que todo submódulo de $W$, contém elementos da forma $\left(\begin{array}{c}\prod\left(q-\alpha_{j}\right)^{m_{j}} \\ 0\end{array}\right),\left(\begin{array}{c}0 \\ \prod\left(q-\alpha_{j}\right)^{m_{j}}\end{array}\right)$, para $m_{j} \in \mathbb{N}$ e $\alpha_{1}, \cdots, \alpha_{l}$ os polos de $G_{1}$. Finalmente mostramos que $W$ é irredutível.

Afirmação 5. Seja $M$ um $A_{1}$-submódulo de $W$ não nulo, então existe $f \in K[q]$ tal que

$$
\left(\begin{array}{l}
f \\
0
\end{array}\right),\left(\begin{array}{l}
0 \\
f
\end{array}\right) \in M .
$$

Demonstração. Sejam $g_{0}, g \in K[q]$ tal que $\left(\begin{array}{c}g_{0} \\ g\end{array}\right) \in M \leq A_{1}\left(\begin{array}{l}1 \\ 0\end{array}\right)$ e $h=\prod\left(q-\alpha_{i}\right)^{l_{i}}$, onde $l_{i}=\max \left\{r_{i}, s_{i}\right\}$, e $h_{\alpha_{i}}=h\left(q-\alpha_{i}\right)^{-l_{i}}$. Assim para $\alpha \in \operatorname{Pol}\left(G_{1}\right), h_{\alpha}$ é o polinômio de grau mínimo relativamente primo $\operatorname{com} q-\alpha$ tal que o único polo de $h_{\alpha} a, h_{\alpha} b$ é $\alpha$. Então

$$
\begin{aligned}
\left(g_{0} h p+\left(b g h-h g_{0}^{\prime}\right)\right)\left(\begin{array}{c}
g_{0} \\
g
\end{array}\right) & =\left(\begin{array}{c}
0 \\
f
\end{array}\right), \\
\left(g h p+\left(a h g-g^{\prime} h-g_{0} h\right)\right)\left(\begin{array}{c}
g_{0} \\
g
\end{array}\right) & =\left(\begin{array}{c}
-f \\
0
\end{array}\right) .
\end{aligned}
$$

Onde $f=g_{0}^{2} h+g_{0} g^{\prime} h-g_{0}^{\prime} g h-g_{0} g a h+g^{2} b h \neq 0$, dado que $N$ é um $\mathfrak{B}$-modulo irredutível de grau dois. 
Lembremos que $G_{1}=p^{2}+a p+b$ é preservado e irredutível, onde $a$ e $b$ são como segue

$$
a=\frac{a_{1}}{\prod\left(q-\alpha_{i}\right)^{r_{i}}}, b=\frac{b_{1}}{\prod\left(q-\alpha_{i}\right)^{s_{i}}} .
$$

Afirmação 6. Suponhamos que o conjunto dos polos de $G_{1}$ está dado por $\operatorname{Pol}\left(G_{1}\right)=$ $\left\{\alpha_{1}, \cdots, \alpha_{l}\right\}$. Então existem $m_{j} \in \mathbb{N}$ tais que $\left(\begin{array}{c}\prod\left(q-\alpha_{j}\right)^{m_{j}} \\ 0\end{array}\right),\left(\begin{array}{c}0 \\ \prod\left(q-\alpha_{j}\right)^{m_{j}}\end{array}\right) \in M$.

Demonstração. Da afirmação anterior $\left(\begin{array}{c}f^{\prime} \\ 0\end{array}\right), p\left(\begin{array}{c}f^{\prime} \\ 0\end{array}\right) \in M$, então

$$
\left(\begin{array}{c}
\prod\left(q-\alpha_{j}\right)^{l_{j}} f^{\prime} \\
0
\end{array}\right)\left(\begin{array}{c}
0 \\
\prod\left(q-\alpha_{j}\right)^{l_{j}} f^{\prime}
\end{array}\right) \in M .
$$

Usando indução, existem $y_{1}, \cdots, y_{l}$ tais que $\left(\begin{array}{c}\prod\left(q-\alpha_{j}\right)^{y_{j}} \\ 0\end{array}\right),\left(\begin{array}{c}0 \\ \prod\left(q-\alpha_{j}\right)^{y_{j}}\end{array}\right) \in M$.

Observação 3. Se $\left(\begin{array}{c}\prod\left(q-\alpha_{j}\right)^{m_{j}} \\ 0\end{array}\right),\left(\begin{array}{c}0 \\ \prod\left(q-\alpha_{j}\right)^{m_{j}}\end{array}\right) \in M$, então

$$
\prod_{j \neq i}\left(q-\alpha_{j}\right) p\left(\begin{array}{c}
\prod\left(q-\alpha_{k}\right)^{m_{k}} \\
0
\end{array}\right) \in M,
$$

para $i=1, \cdots, l, \log 0\left(\begin{array}{c}\prod\left(q-\alpha_{i}\right)^{m_{i}-1} \\ 0\end{array}\right) \in M$.

Teorema 24. Seja $F \in M n n A n n(N)$, então o módulo $W=S_{o c} c_{A_{1}}(N)$ é irredutivel e da seguente forma

1. $W=A_{1}\left(\begin{array}{l}1 \\ 0\end{array}\right)$ se, e somente se, F é preservado.

2. $W=A_{1}\left(\begin{array}{c}\prod_{\alpha \in \Delta(F)}(q-\alpha)^{n_{\alpha}} \\ 0\end{array}\right)$ se, e somente se, $Q_{\alpha, F}\left(-n_{\alpha}\right)=0$ para $0<n \in \mathbb{N} e$ $\alpha \in \Delta(F)$.

Demonstração. Sejam $m_{1}, \cdots, m_{l}$ inteiros positivos com $\sum m_{j}$ mínima tais que $\left(\begin{array}{c}\prod\left(q-\alpha_{j}\right)^{m_{j}} \\ 0\end{array}\right),\left(\begin{array}{c}0 \\ \prod\left(q-\alpha_{j}\right)^{m_{j}}\end{array}\right) \in M$. Da observação anterior $\left(\begin{array}{c}\prod\left(q-\alpha_{i}\right)^{m_{i}-1} \\ 0\end{array}\right) \in M$. Basta mostrar que $\left(\prod_{j \neq i}\left(q-\alpha_{j}\right)^{m_{j}}\left(q-\alpha_{i}\right)^{m_{i}-1}\right) \in M$.

De $p\left(\begin{array}{c}0 \\ \prod\left(q-\alpha_{j}\right)^{m_{j}}\end{array}\right)$ e $p\left(\begin{array}{c}\prod\left(q-\alpha_{j}\right)^{m_{j}-1} \\ 0\end{array}\right)$ temos os seguintes elementos de $M$

$$
\begin{aligned}
\left(\begin{array}{c}
-\operatorname{Res}_{\alpha}(b) \prod_{j \neq i}\left(q-\alpha_{j}\right)^{m_{j}}\left(q-\alpha_{i}\right)^{m_{i}-s_{i}} \\
\left(m_{j}-\operatorname{Res}_{\alpha}(a) \prod_{j \neq i}\left(q-\alpha_{j}\right)\left(q-\alpha_{i}\right)^{1-r_{i}}\right) \prod\left(q-\alpha_{j}\right)^{m_{j}-1}
\end{array}\right) & \in M \\
\left(\begin{array}{c}
\left(m_{i}-1\right) \prod_{j \neq i}\left(q-\alpha_{j}\right)^{m_{j}-1}\left(q-\alpha_{i}\right)^{m_{i}-2} \\
\prod_{j \neq i}\left(q-\alpha_{j}\right)^{m_{j}}\left(q-\alpha_{i}\right)^{m_{i}-1}
\end{array}\right) & \in M .
\end{aligned}
$$


Usando os elementos acima, mostraremos que

$$
\left(\begin{array}{c}
0 \\
\left.Q_{\alpha, G_{1}}\left(-m_{i}\right) \prod_{j \neq i}\left(q-\alpha_{j}\right)^{m_{j}}\left(q-\alpha_{i}\right)^{m_{i}-1}\right) \in M
\end{array}\right.
$$

em consequência, $\left(\begin{array}{c}0 \\ \prod\left(q-\alpha_{j}\right)^{m_{j}-1}\end{array}\right) \in M$. Portanto $W$ é irredutível e tem a estrutura do enunciado.

A demonstração de (2.14) depende da forma do polinômio $Q_{\alpha, G_{1}}(a)$, ver (2.1).

1. Se $s_{i}=1$ e $r_{i}=0$, então $Q_{\alpha_{i}, G_{1}}(\xi)=\xi(\xi-1)$. De (2.12) temos

$$
\left(m_{i}\left(m_{i}+1-\operatorname{Res}_{\alpha}(a)\right) \prod_{j \neq i}^{0}\left(q-\alpha_{j}\right)^{m_{j}}\left(q-\alpha_{i}\right)^{m_{i}-1}\right) \in M .
$$

2. Suponhamos que $s_{i}=2$ e $r_{i}=0$, então $Q_{\alpha_{i}, G_{1}}(\xi)=\xi(\xi-1)+\operatorname{Res}_{\alpha}(b)$. De (2.12) temos

$$
\left(\left(m_{i}\left(m_{i}-1\right)+\operatorname{Res}_{\alpha}(b)\right) \prod_{j \neq i}^{0}\left(q-\alpha_{j}\right)^{m_{j}}\left(q-\alpha_{i}\right)^{m_{i}-1}\right) \in M .
$$

3. Se $s_{i} \leq 1$ e $r_{i}=1$. Então $Q_{\alpha_{i}, G_{1}}(\xi)=\xi\left(\xi-1+\operatorname{Res}_{\alpha}(a)\right)$. De (2.12) temos

$$
\left(m_{i}\left(m_{i}+1-\operatorname{Res}_{\alpha}(a)\right) \prod_{j \neq i}^{0}\left(q-\alpha_{j}\right)^{m_{j}}\left(q-\alpha_{i}\right)^{m_{i}-1}\right) \in M
$$

4. Se $s_{i}=2$ e $r_{i}=1$, então $Q_{\alpha_{i}, G_{1}}(\xi)=\xi(\xi-1)+\operatorname{Res}_{\alpha}(a) \xi+\operatorname{Res}_{\alpha}(b)$. De (2.12) temos

$$
\left(\left(m_{i}\left(m_{i}-1\right)+m_{i} \operatorname{Res}_{\alpha}(a)+\operatorname{Res}_{\alpha}(b)\right) \prod_{j \neq i}\left(q-\alpha_{j}\right)^{m_{j}}\left(q-\alpha_{i}\right)^{m_{i}-1}\right) \in M .
$$

5. Nos casos restantes a demonstração é similar.

\subsubsection{Se $\Delta\left(G_{1}\right)=\emptyset$}

Neste caso todos os polos de $G_{1}$ são essenciais, então $G_{1}=F_{1}$. Lembremos que $u=\left(\begin{array}{l}1 \\ 0\end{array}\right)$. A seguintes afirmações são usadas para construir exemplos de polinômios irredutíveis em $\mathfrak{B}$.

Afirmação 7. O polinômio $F_{1}=a_{2} p^{2}+a_{1} p+a_{0} \in A_{1}$ é irredutível se, e somente se, a equação diferencial $a_{2} z^{\prime \prime}+a_{1} z^{\prime}+a_{0} z=0$ não tem soluções em $K(q)$. (Método de Frobenious).

Teorema 25. Seja $F=\sum_{i=0}^{m} f_{i} p^{i} \in A_{1}$. Se existe $\alpha \in K$ tal que $q-\alpha$ divide a $f_{i}$ para $i \geq 1, q-\alpha$ não divide a $f_{0} e(q-\alpha)^{2}$ não divide a $f_{m}$, então $F$ é irredutível em $\mathfrak{B}$.

Demonstração. Ver (Kovacic, 1972). 
Exemplo 4. Seja $F_{1}=p^{2}+p+\frac{1}{(q-\alpha)}$, onde $\alpha \in K$. Pelo teorema anterior o polinômio $F_{1}$ é irredutível, e que $Q_{\alpha, F_{1}}(\xi)=\xi(\xi-1)$.

Veremos que $W \varsubsetneqq K\left[q,(q-\alpha)^{-1}\right] \times K\left[q,(q-\alpha)^{-1}\right]$. Dado que $W$ é irredutível $w \in W$ se, e somente se, existem $f_{i}(q) \in K[q]$ tal que $w=\sum f_{i}(q) p^{i} u$. Por outra parte os seguinte elementos pertencem $W$

$$
\begin{aligned}
& p^{2} u \Rightarrow w_{0}=(q-\alpha)^{-1} u \in W, p^{2} u \in\left\langle u, p u, w_{0}\right\rangle_{K} . \\
& p^{3} u \Rightarrow w_{1}=(q-\alpha)^{-1} u-(q-\alpha)^{-2} p u \in W, p^{3} u \in\left\langle u, p u, w_{0}, w_{1}\right\rangle_{K} . \\
& p^{4} u \Rightarrow w_{2}=\left(\begin{array}{c}
2(q-\alpha)^{-3}-(q-\alpha)^{-2} \\
-2(q-\alpha)^{-2}-(q-\alpha)^{-1}
\end{array}\right) \in W, p^{4} u \in\left\langle u, p u, w_{0}, w_{1}, w_{2}\right\rangle_{K} .
\end{aligned}
$$

Provaremos que para cada $n \in \mathbb{N}, p^{n} u \in\left\langle u, p u, w_{0}, \cdots, w_{n}\right\rangle_{K}$, e $(q-\alpha) w_{j} \in\left\langle u, p u, w_{0}, \cdots, w_{j-1}\right\rangle_{K}$, para $j \leq n$. Então $W$ e gerado por

$$
\left\{u, p u, w_{0}, \cdots, w_{n}, \cdots\right\}_{K[q]}
$$

deste conjunto gerador temos $\left(\begin{array}{c}0 \\ (q-\alpha)^{-1}\end{array}\right) \notin W$. Suponhamos que a hipótese é verdadeira para $n$. Definimos $w_{n+1}=p w_{n}$, usando a identidade $(q-\alpha) p=p(q-\alpha)+1$ e a hipóteses de indução temos o conjunto $\left\{u, p u, w_{0}, \cdots, w_{n+1}\right\}_{K[q]}$, que satisfaz as hipóteses do enunciado. Assim, provamos que $W \varsubsetneqq K\left[q,(q-\alpha)^{-1}\right] \times K\left[q,(q-\alpha)^{-1}\right]$.

Lema 19. Se $\Delta\left(F_{1}\right)=\varnothing$, então $W=K_{P o l\left(F_{1}\right)} \times K_{P o l\left(F_{1}\right)}$.

Demonstração. Sabemos que $W$ é um $A_{1}$-módulo irredutível. Se o polinômio principal não tem raízes inteiras, então ele está identificado com um dos polinômios da seguinte lista;

$$
Q_{\alpha, F_{1}}(\xi)= \begin{cases}(\xi-1) \xi+\operatorname{Res}_{\alpha}(b) & \nu_{\alpha}(a)=0, \nu_{\alpha}(b)=-2 . \\ (\xi-1) \xi+\operatorname{Res}_{\alpha}(a) \xi+\operatorname{Res}_{\alpha}(b) & \nu_{\alpha}(a)=-1, \nu_{\alpha}(b)=-2 . \\ \operatorname{Res}_{\alpha}(a) \xi+\operatorname{Res}_{\alpha}(b) & \nu_{\alpha}(a) \leq-2, \nu_{\alpha}(b)-1=\nu_{\alpha}(a) \\ \operatorname{Res}_{\alpha}(b) & \nu_{\alpha}(b) \leq-3, \nu_{\alpha}(a)<\nu_{\alpha}(b)-1 .\end{cases}
$$

Seja $k$ o menor inteiro positivo tal que $\left(\begin{array}{c}(q-\alpha)^{-k} \\ 0\end{array}\right)$, e $\left(\begin{array}{c}0 \\ (q-\alpha)^{-k+1}\end{array}\right) \in W$, , então

$$
\left(\begin{array}{c}
-k(q-\alpha)^{-k-1} \\
(q-\alpha)^{-k}
\end{array}\right) \text { e }\left(\begin{array}{c}
-b_{1}(q-\alpha)^{-s_{\alpha}-k+1} \prod_{\alpha_{i} \neq \alpha}\left(q-\alpha_{i}\right)^{-s_{i}+l_{i}} \\
-(k-1)(q-\alpha)^{-k}-a_{1}(q-\alpha)^{-r_{\alpha}-k+1} \prod_{\alpha_{i} \neq \alpha}\left(q-\alpha_{i}\right)^{-s_{i}}
\end{array}\right)
$$

são elementos de $W$. Na demonstração usamos a identificação $r_{\alpha}=-\nu_{\alpha}(b)$ e $-\nu_{\alpha}(a)=s_{\alpha}$.

1. Suponhamos que $r_{\alpha}=0, s_{\alpha}=2$. De (2.16), temos $\left(\begin{array}{c}-\operatorname{Res}_{\alpha}(b)(q-\alpha)^{-1-k} \\ -(k-1)(q-\alpha)^{-k}\end{array}\right) \in W$, portanto $\left(\begin{array}{c}Q_{\alpha, F_{1}}(k+1)(q-\alpha)^{-k-1} \\ 0\end{array}\right) \in W$. Dado que $Q_{\alpha, F_{1}}(n) \neq 0$ se $n \in \mathbb{N}$, podemos concluir que $\left(\begin{array}{c}(q-\alpha)^{-n} \\ 0\end{array}\right) \in W$ e $K\left[q,(q-\alpha)^{-1}\right] \times K\left[q,(q-\alpha)^{-1}\right] \subset W$. 
2. Suponhamos que $r_{\alpha}=1, s_{\alpha}=2$. Multiplicando (2.16) por $h_{\alpha}$ tem-se

$$
\left(\begin{array}{c}
\operatorname{Res}_{\alpha}(b)\left(q-\alpha_{1}\right)^{-k-1} \\
-\left(k-1-\operatorname{Res}_{\alpha}(a)\right)(q-\alpha)^{-k}
\end{array}\right) \in W
$$

portanto $\left(\begin{array}{c}Q_{\alpha, F_{1}}(k+1)\left(q-\alpha_{1}\right)^{-k-1} \\ 0\end{array}\right) \in W$. Dado que $Q_{\alpha, F_{1}}(n) \neq 0$, para $n \in \mathbb{N}$, obtemos que $\left(\begin{array}{c}(q-\alpha)^{-n} \\ 0\end{array}\right) \in W$ e portanto $K\left[q,(q-\alpha)^{-1}\right] \times K\left[q,(q-\alpha)^{-1}\right] \subset W$.

3. Suponhamos que $r_{\alpha} \geq 2, s_{\alpha}=r_{\alpha}+1$. Multiplicando (2.16) por $h_{\alpha}$ temos

$$
\left(\begin{array}{c}
\operatorname{Res}_{\alpha}(b)(q-\alpha)^{-k-r_{\alpha}} \\
\left(-(k-1)(q-\alpha)^{-k}-\operatorname{Res}_{\alpha}(a)\right)(q-\alpha)^{-k-r_{\alpha}+1}
\end{array}\right) \in W
$$

multiplicando por $(q-\alpha)^{r_{\alpha}-1}$ o elemento anterior, obtemos $\left(\begin{array}{c}Q_{\alpha, F_{1}}(k+1)(q-\alpha)^{-k-1} \\ 0\end{array}\right) \in$ $W$. Dado que $Q_{\alpha, F_{1}}(n) \neq 0$ para $n \in \mathbb{N}$, temos $\left(\begin{array}{c}(q-\alpha)^{-n} \\ 0\end{array}\right) \in W$. Portanto

$$
K\left[q,(q-\alpha)^{-1}\right] \times K\left[q,(q-\alpha)^{-1}\right] \subset W .
$$

4. Suponhamos que $s_{\alpha} \geq 3$, então $s_{\alpha}>r_{\alpha}+1$. Multiplicando (2.16) por $h_{\alpha}$ obtemos

$$
\left(\begin{array}{c}
\operatorname{Res}_{\alpha}(b)(q-\alpha)^{-k-s_{\alpha}+1} \\
\left.-(k-1)(q-\alpha)^{-k}-\operatorname{Res}_{\alpha}(a)\right)(q-\alpha)^{-k-r_{\alpha}+1}
\end{array}\right) \in W
$$

multiplicando por $(q-\alpha)^{s_{\alpha}-1}$ o elemento anterior, obtemos $\left(\begin{array}{c}\operatorname{Res}_{\alpha}(b)(q-\alpha)^{-k-1} \\ 0\end{array}\right)$, e dado que $Q_{\alpha, F_{1}}(n) \neq 0$ para $n \in \mathbb{N}$, segue-se

$$
\left(\begin{array}{c}
(q-\alpha)^{-n} \\
0
\end{array}\right) \in W \text { e } K\left[q,(q-\alpha)^{-1}\right] \times K\left[q,(q-\alpha)^{-1}\right] \subset W
$$

Portanto, para cada $\alpha \in \operatorname{Pol}\left(F_{1}\right)$ temos $K\left[q,(q-\alpha)^{-1}\right] \times K\left[q,(q-\alpha)^{-1}\right] \subset W$, então

$$
W=K\left[q,\left(q-\alpha_{1}\right)^{-1}, \cdots,\left(q-\alpha_{l}\right)^{-1}\right] \times K\left[q,\left(q-\alpha_{1}\right)^{-1}, \cdots,\left(q-\alpha_{l}\right)^{-1}\right] .
$$

\subsubsection{Se $\Delta\left(G_{1}\right) \neq \emptyset$}

Determinaremos que polos de $G_{1}$ são essenciais. Denotaremos o polinômio reduzido, ver difinição 19 , obtido de $G_{1}$ tirando os polos não essenciais por $F_{1}$. Da seção anterior, se um polos $\alpha$ de $G_{1}$ não é essencial, então $\alpha \in \Delta\left(G_{1}\right)$.

Na forma do polinômio principal $Q_{\alpha, G_{1}}(\xi)$ que foi dada em (2.3), para $\alpha \in \Delta\left(G_{1}\right)$, substituímos $r_{\alpha}=-\nu_{\alpha}(b)$ e $-\nu_{\alpha}(a)=s_{\alpha}$. O polinômio principal $Q_{\alpha, G_{1}}(\xi)$ associado a $G_{1}$ no polo 
$\alpha$ é da seguinte forma

$$
Q_{\alpha, G_{1}}(\xi)= \begin{cases}(\xi-1) \xi & r_{\alpha}=0, s_{\alpha}=1 \\ (\xi-1) \xi+\operatorname{Res}_{\alpha}(a) \xi & r_{\alpha}=1, s_{\alpha} \leq 1 \\ \operatorname{Res}_{\alpha}(a) \xi & r_{\alpha} \geq 2, r_{\alpha} \geq s_{\alpha}\end{cases}
$$

Para determinar a unicidade de $W$ salvo isomorfismos e a sua estrutura, precisamos saber quando um polo $\alpha \in \operatorname{Pol}\left(G_{1}\right)$ é essencial.

Observação 4. Seja $u=\left(\begin{array}{l}1 \\ 0\end{array}\right), p u=\left(\begin{array}{l}0 \\ 1\end{array}\right)$ e $l_{\alpha}=\max \left\{r_{\alpha}, s_{\alpha}\right\}$.

Se $(q-\alpha)^{-t} u \in W$ então $(q-\alpha)^{-t+1} p u \in W$, assim

$$
p(q-\alpha)^{-t+1} u=(q-\alpha)^{-t+1} p u+(-t+1)(q-\alpha)^{-t} u \in W,
$$

$\operatorname{logo}(q-\alpha)^{-t+1} p u \in W$. Além disso, se $(q-\alpha)^{-t} u \in W$ para cada $t \in \mathbb{N}$ então

$$
K\left[q,(q-\alpha)^{-1}\right] \times K\left[q,(q-\alpha)^{-1}\right] \subset W .
$$

Seja $\alpha \in \Delta\left(G_{1}\right)$. Da equação $p^{2} u+a p u+b u=0$, temos os seguentes elementos de $W$

$$
\begin{aligned}
w_{1}=p(q-\alpha)^{-t} u & =(q-\alpha)^{-t} p u-t(q-\alpha)^{-t-1} u, \\
w_{2}=p^{2}(q-\alpha)^{-t} u & =-(a+2 t)(q-\alpha)^{-t-1} p u+\left(t(t+1)-b(q-\alpha)^{2}\right)(q-\alpha)^{-t-2} u .
\end{aligned}
$$

De $t(q-\alpha) w_{2}+\left(t(t+1)-b(q-\alpha)^{2}\right) w_{1}$ obtemos o elemento

$$
w_{3}=\left(t(t-1)+a t(q-\alpha)+b\left(q-\alpha^{2}\right)\right)(q-\alpha)^{-t} p u .
$$

De $w_{1}, w_{3}$ segue-se $(q-\alpha)^{-1-t} u \in W$. Mostraremos que para $t \in \mathbb{N}$ tal que $Q_{\alpha, G_{1}}(t) \neq 0$ temos $(q-\alpha)^{-t} u,(q-\alpha)^{-t+1} p u \in W$.

Da observação (2), Para $\alpha \in \operatorname{Pol}\left(G_{1}\right)$ tal que $\alpha$ não é essencial temos

1. $\nu_{\alpha}(a)=-1, \nu_{\alpha}(b) \geq-1$ e $\operatorname{Res}_{\alpha}(a)=-n \in \mathbb{Z}$.

2. $\nu_{\alpha}(b)=-1$ e $\nu_{\alpha}(a) \geq 0$

Os polos que satisfazem as condições acima, são os polos que ainda não sabemos quando são essenciais. De $h_{\alpha} p^{k+2} u=\left(\begin{array}{l}x_{k+1} \\ y_{k+2}\end{array}\right)$ definimos $w_{k}=\left(\begin{array}{l}x_{k+1}^{-} \\ y_{k+2}^{-}\end{array}\right)$, onde $x_{k+1}^{-}$é a soma dos monômios de $x_{k+1}$ tal que $\nu_{\alpha}$ é negativo. Da mesma forma definimos $y_{k+1}^{-}$. Usando indução construimos os elementos $w_{k}$, dista construção $W \cap K\left[q,(q-\alpha)^{-1}\right] \times K\left[q,(q-\alpha)^{-1}\right]$ é gerado por $T(\alpha) \cup\{u, p u\}$ sobre $K[q]$ onde

$$
T(\alpha)=\left\{w_{k} \mid k \in \mathbb{N}\right\}
$$

Observação 5. 1. Suponhamos que $\nu_{\alpha}(a)=-1$ e $\nu_{\alpha}(b) \geq 0$. Construimos por indução os elementos $w_{k}$ a partir de $p^{k+1}\left(\begin{array}{l}1 \\ 0\end{array}\right)$ como segue

$$
w_{k}=\left(\begin{array}{c}
-(k-1)(q-\alpha)^{-n-k+\delta}+(q-\alpha)^{-n+1+\delta} l_{k}\left((q-\alpha)^{-1}\right) \\
(q-\alpha)^{-n-k}+(q-\alpha)^{-n} l_{k}^{\prime}\left((q-\alpha)^{-1}\right)
\end{array}\right)
$$


onde $\operatorname{grau}\left(l_{k}\right) \leq k$ e $\operatorname{grau}\left(l_{k}^{\prime}\right) \leq k$ e $\delta=\left|\nu_{\alpha}(b)+\nu_{\alpha}(\delta)\right|$. Portanto, $\left(\begin{array}{c}(q-\alpha)^{-1} \\ 0\end{array}\right) \notin W$ e

$$
K\left[q,(q-\alpha)^{-1}\right] \times K\left[q,(q-\alpha)^{-1}\right] \subset W_{\alpha}=A_{1}\left(\begin{array}{c}
(q-\alpha)^{-1} \\
0
\end{array}\right)
$$

Assim $W_{\alpha} / W$ é irredutível de $S$-torção isomorfo a $M(\alpha)$.

Usando a ação de $p$ em $\left(\begin{array}{l}1 \\ 0\end{array}\right)$ temos

(a) Se $\nu_{\alpha}(b)=t$ para $n-3 \leq t \leq n-1$ então $\operatorname{dim}_{K}(T(\alpha))$ é infinito enumerável.

(b) Se $\nu_{\alpha}(b) \geq n$, então $\operatorname{dim}_{K}(T(\alpha))=n$.

2. Suponhamos que $\nu_{\alpha}(a)=-1$ e $\nu_{\alpha}(b)=-1$. Se $p^{n+2}\left(\begin{array}{l}1 \\ 0\end{array}\right)$ pertence ao gerado por $p^{j}\left(\begin{array}{l}1 \\ 0\end{array}\right), j=0,1, \cdots, n+1$ sobre $K[q]$ então $p^{n+2+i}\left(\begin{array}{l}1 \\ 0\end{array}\right)$ também pertence. Portanto, $T(\alpha)=\left\{w_{k} \mid k=1, \cdots, n\right\}$. Os $w_{k}$ são construidos por indução a partir de $p^{k+1}\left(\begin{array}{l}1 \\ 0\end{array}\right)$. $w_{1}=\left(\begin{array}{l}\operatorname{Res}_{\alpha}(b)(q-\alpha)^{-1} \\ \operatorname{Res}_{\alpha}(a)(q-\alpha)^{-1}\end{array}\right)$

$$
\begin{gathered}
w_{2}=\left(\begin{array}{c}
\operatorname{Res}_{\alpha}(b)\left(1+\operatorname{Res}_{\alpha}(a)\right)(q-\alpha)^{-2}+\beta_{1} \operatorname{Res}_{\alpha}(a)(q-\alpha)^{-1} \\
\operatorname{Res}_{\alpha}(a)\left(1+\operatorname{Res}_{\alpha}(a)\right)(q-\alpha)^{-2}+\left(\mu_{1} \operatorname{Res}_{\alpha}(a)-\operatorname{Res}_{\alpha}(b)\right)(q-\alpha)^{-1}
\end{array}\right) \\
w_{k+1}=\left(\begin{array}{c}
t_{k, 0}(q-\alpha)^{-k-1}+t_{k, 1}(q-\alpha)^{-k}+f_{k+1}\left((q-\alpha)^{-1}\right) \\
t_{k, 0}^{\prime}(q-\alpha)^{-k-1}+t_{k, 1}^{\prime}(q-\alpha)^{-k}+g_{k+1}\left((q-\alpha)^{-1}\right)
\end{array}\right) .
\end{gathered}
$$

Onde $\operatorname{grau}\left(f_{k+1}\right), \operatorname{grau}\left(g_{k+1}\right) \leq k$ e $t_{k, 0}, t_{k, 1}, t_{k, 0}^{\prime}, t_{k, 1}^{\prime}$ estão dados por

$$
\begin{aligned}
& t_{k, 0}=\operatorname{Res}_{\alpha}(b) \prod_{i=1}^{k}\left(i+\operatorname{Res}_{\alpha}(a)\right) \\
& t_{k, 1}=\left(b_{\alpha, 1} \operatorname{Res}_{\alpha}(a)\left(k+\operatorname{Res}_{\alpha}(a)\right) \prod_{i=2}^{k-1}\left(i+\operatorname{Res}_{\alpha}(a)\right)\right. \\
& \left.+(k-1) \operatorname{Res}_{\alpha}(b)\left(\mu_{1} \operatorname{Res}_{\alpha}(a)-\operatorname{Res}_{\alpha}(b)\right)\right) \prod_{i=2}^{k-1}\left(i+\operatorname{Res}_{\alpha}(a)\right) \\
& t_{k, 0}^{\prime}=\operatorname{Res}_{\alpha}(a) \prod_{i=1}^{k}\left(i+\operatorname{Res}_{\alpha}(a)\right) \\
& t_{k, 1}^{\prime}=k\left(\mu_{1} \operatorname{Res}_{\alpha}(a)-\operatorname{Res}_{\alpha}(b)\right) \prod_{i=1}^{k-1}\left(i+\operatorname{Res}_{\alpha}(a)\right) .
\end{aligned}
$$


Se $n=-\operatorname{Res}_{\alpha}(a) \in \mathbb{N}$ então o conjunto $W \cap K\left[q,(q-\alpha)^{-1}\right] \times K\left[q,(q-\alpha)^{-1}\right]$ é gerado por $T(\alpha)=\left\{w_{k} \mid k \in \mathbb{N}\right\} \cup\{u, p u\}$ sobre $K[q]$. Se $\operatorname{dim}_{k}(T(\alpha))=n$ temos a cadeia de $A_{1}$-módulos

$$
W \subset V_{\alpha}=A_{1}\left(\begin{array}{c}
(q-\alpha)^{-1} \\
0
\end{array}\right) \subset W_{\alpha}=A_{1}\left(\begin{array}{c}
(q-\alpha)^{n-2} \\
0
\end{array}\right)
$$

Pela estrutura de $W$ e a ação de $p$ os quocientes $V_{\alpha} / W$ e $W_{\alpha} / V_{\alpha}$ são irredutíveis, de $S$-torção e portanto isomorfos a $M(\alpha)$. Se $\operatorname{dim}_{K}(T(\alpha))$ é infinito enumerável, temos $W=V_{\alpha}$.

Se $\operatorname{Res}_{\alpha}(a) \notin \mathbb{Z}^{-}$, então $\left(\begin{array}{c}(q-\alpha)^{-1} \\ 0\end{array}\right) \in W$ e $\left(\begin{array}{c}(q-\alpha)^{-t-2} \\ 0\end{array}\right) \notin W, \log \mathrm{o}$

$$
K\left[q,(q-\alpha)^{-1}\right] \times K\left[q,(q-\alpha)^{-1}\right] \subset W_{\alpha}=A_{1}\left(\begin{array}{c}
(q-\alpha)^{-t-2} \\
0
\end{array}\right) .
$$

Portanto, $W_{\alpha} / W$ é irredutível de $S$-torção isomorfa a $M(\alpha)$.

Denotaremos por $T(\alpha)$ o conjunto conformado pelos elemento $w_{j}$.

3. Suponhamos que $\nu_{\alpha}(b)=-1$ e $\nu_{\alpha}(a) \geq 0$. Os elementos $w_{k}$ estão dados por

$$
w_{k}=\left(\begin{array}{c}
-(k-1)(q-\alpha)^{-k}+l_{k}\left((q-\alpha)^{-1}\right) \\
(q-\alpha)^{-k+1}+l_{k}^{\prime}\left((q-\alpha)^{-1}\right)
\end{array}\right) .
$$

onde $l_{k}, l_{k}^{\prime} \in K[q] \operatorname{com} \operatorname{grau}\left(l_{k}\right)=k-1, \operatorname{grau}\left(l_{k}^{\prime}\right)=k-2$. Denotaremos por $T(\alpha)$ o conjunto conformado pelos elemento $w_{j}$, assim $\left(\begin{array}{c}(q-\alpha)^{-2} \\ 0\end{array}\right) \notin W$ e

$$
K\left[q,(q-\alpha)^{-1}\right] \times K\left[q,(q-\alpha)^{-1}\right] \subset W_{\alpha}=A_{1}\left(\begin{array}{c}
(q-\alpha)^{-2} \\
0
\end{array}\right) .
$$

Portanto, $W_{\alpha} / W$ é irredutível de $S$-torção isomorfa a $M(\alpha)$.

4. Suponhamos que $\nu_{\alpha}(b) \geq \nu_{\alpha}(a)$ e $\nu_{\alpha}(a) \leq-2$. Seja $\delta=\nu_{\alpha}(b)-\nu_{\alpha}(a)$ então os elementos $w_{k}$ estão dados por

$$
w_{k}=\left(\begin{array}{c}
\operatorname{Res}_{\alpha}\left((q-\alpha)^{-\nu_{\alpha}(b)-1} b\right)(q-\alpha)^{-k}+l_{k}\left((q-\alpha)^{-1}\right) \\
\operatorname{Res}_{\alpha}\left((q-\alpha)^{-\nu_{\alpha}(a)-1} a\right)(q-\alpha)^{-k-t}+l_{k}^{\prime}\left((q-\alpha)^{-1}\right)
\end{array}\right) .
$$

onde $l_{k}, l_{k}^{\prime} \in K[q] \operatorname{com} \operatorname{grau}\left(l_{k}\right)=k-1$ e $\operatorname{grau}\left(l_{k}^{\prime}\right)=-k-\delta+1, k \in \mathbb{N}$. Se $\nu_{\alpha}(b)>\nu_{\alpha}(a)$ substituímos $b_{\alpha, i}$ por $(q-\alpha)^{\nu_{\alpha}(b)-\nu_{\alpha}(a)} b_{\alpha, i}$. Denotaremos por $T(\alpha)$ o conjunto conformado pelos elemento $w_{j}$. Assim $\left(\begin{array}{c}(q-\alpha)^{-1} \\ 0\end{array}\right) \notin W$ e

$$
K\left[q,(q-\alpha)^{-1}\right] \times K\left[q,(q-\alpha)^{-1}\right] \subset W_{\alpha}=A_{1}\left(\begin{array}{c}
(q-\alpha)^{-1} \\
0
\end{array}\right) .
$$

Portanto, $W_{\alpha} / W$ é irredutível de $S$-torção isomorfa a $M(\alpha)$. 
Lema 20. Seja $\alpha \in \Delta\left(G_{1}\right)$ com polinômio principal $Q_{\alpha, G_{1}}(\xi)$, como em (2.3). Se Res $s_{\alpha}(a) \in$ $\mathbb{Z}^{-}$, então $(q-\alpha)^{\operatorname{Res}_{\alpha}(a)-2} u \notin W$. Se $\operatorname{Res}_{\alpha}(a) \notin \mathbb{Z}^{-}$, então $\left(\begin{array}{c}(q-\alpha)^{-1} \\ 0\end{array}\right) \notin W$.

Demonstração. 1. Suponhamos que $r_{\alpha}=1, s_{\alpha}=0$, 1, ver (2.5). De (2.19) temos

$$
t\left(\operatorname{Res}_{0}(a)-1+t\right)(q-\alpha)^{-1-t} u=Q_{\alpha, G_{1}}(t)(q-\alpha)^{-1-t} u \in W .
$$

Dado que $(q-\alpha)^{-1} u$ ou $(q-\alpha)^{\operatorname{Res}_{\alpha}(a)-2} u$ não pertencem ao gerado por $T(\alpha) \cup\{u, p u\}$ sobre $K[q]$, então $W \subseteq A_{1}\left(\begin{array}{c}(q-\alpha)^{-1} \\ 0\end{array}\right) \subset W_{\alpha}=A_{1}\left(\begin{array}{c}(q-\alpha)^{\operatorname{Res}_{\alpha}(a)-2} \\ 0\end{array}\right)$. Além disso

$$
K\left[q,(q-\alpha)^{-1}\right] \times K\left[q,(q-\alpha)^{-1}\right] \subset W_{\alpha}=A_{1}\left(\begin{array}{c}
(q-\alpha)^{\operatorname{Res} \alpha(a)-2} \\
0
\end{array}\right)
$$

2. Suponhamos que $r_{\alpha}=0, s_{\alpha}=1$, ver (2.5). Como $(q-\alpha)^{-2} u$ não está no gerado por $T(\alpha) \cup\{u, p u\}$ sobre $K[q]$, então

$$
W \subset W_{\alpha}=A_{1}\left(\begin{array}{c}
(q-\alpha)^{-2} \\
0
\end{array}\right)
$$

Além disso, do Lema $(20), K\left[q,(q-\alpha)^{-1}\right] \times K\left[q,(q-\alpha)^{-1}\right] \subset W_{\alpha}$.

3. Suponhamos que $r_{\alpha} \geq 2, r_{\alpha} \geq s_{\alpha}-1$. Seja $k_{\alpha}=r_{\alpha}-s_{\alpha}$ então $v=(q-\alpha)^{-k_{\alpha}} p u \in W$. Seja $t$ o maior inteiro tal que $(q-\alpha)^{-t} u \in W$. De $(a(q-\alpha)+2 t) p(q-\alpha)^{-t} u+(q-\alpha) w_{2}$, temos $(q-\alpha)^{-t-1} u \in W$. Como $(q-\alpha)^{-1} u$ não está no gerado por $T(\alpha) \cup\{u, p u\}$ sobre $K[q]$, então

$$
W \subset W_{\alpha}=A_{1}\left(\begin{array}{c}
(q-\alpha)^{-1} \\
0
\end{array}\right)
$$

Além disso, do Lema $(20), K\left[q,(q-\alpha)^{-1}\right] \times K\left[q,(q-\alpha)^{-1}\right] \subset W_{\alpha}$.

\subsection{Relação entre os módulos $W$ e $K_{P o l\left(F_{1}\right)} \times K_{P o l\left(F_{1}\right)}$}

Do anterior, se $\operatorname{dim}_{K}(T(\alpha))$ é finita então $\operatorname{dim}_{K}(T(\alpha))=-\operatorname{Res}_{\alpha}(a)$ e

$$
W=A_{1}\left(\begin{array}{l}
1 \\
0
\end{array}\right) \subseteq V_{\alpha}=A_{1}\left(\begin{array}{c}
(q-\alpha)^{-1} \\
0
\end{array}\right) \subset A_{1}\left(\begin{array}{c}
(q-\alpha)^{-2+\operatorname{Res}_{\alpha}(a)} \\
0
\end{array}\right)
$$

No seguinte lema demonstramos formalmente que $(q-\alpha)^{\operatorname{Res}_{\alpha}(a)-2} u \notin W$, como se mostrou na observação anterior. Lembremos que $-\nu_{\alpha}(a)=r_{\alpha} \mathrm{e}-\nu_{\alpha}(b)=s_{\alpha}$.

Em todos os casos anteriores foi mostrado que $\left(\begin{array}{c}(q-\alpha)^{-\theta_{\alpha}\left(G_{1}\right)-3} \\ 0\end{array}\right) \notin W$, onde $-\theta_{\alpha}\left(G_{1}\right)$ é menor raiz inteira de $Q_{\alpha, G_{1}}(\xi)$. 
As seguintes observações estão nós encaminhando para o nosso objetivo. O qual consiste em mostrar que para todo $\mathfrak{B}$-módulo irredutível $N$ e o polinômio $F \in \operatorname{Mnn} \operatorname{Ann}(N)$ podemos construir $F_{1}$ similar a $F$ tal que todos os polos de $F_{1}$ são essenciais, e assim finalmente determinar uma estrutura para $W=A_{1}\left(\begin{array}{l}1 \\ 0\end{array}\right)$, única salvo isomorfismos.

Observação 6. 1. Seja $\varphi: W \longrightarrow K_{P o l\left(G_{1}\right)-\{\alpha\}} \times K_{P o l\left(G_{1}\right)-\{\alpha\}}$ um homomorfismo injetor de $A_{1}$-módulos. Então existe $f \in K[q]$ não nulo relativamente primo com $q-\alpha$ tal que

$$
\varphi\left(\left(\begin{array}{l}
0 \\
f
\end{array}\right)\right) \in K[q] \times K[q] .
$$

Portanto, para $w_{1} \in T(\alpha)$ temos $\varphi\left(w_{1}\right) \in K[q] \times K[q]$, usando indução obtemos $\varphi\left(w_{n}\right) \in K[q] \times K[q]$ para todo $n \in \mathbb{N}$.

2. Suponhamos que existe $\varphi: W \longrightarrow K_{P o l\left(G_{1}\right)-\{\alpha\}} \times K_{P o l\left(G_{1}\right)-\{\alpha\}}$ um homomorfismo injetor de $A_{1}$-módulos. do item anterior temos $\varphi(K[q] T(\alpha))=L$, onde $L$ é um $K[q]$ submódulo de $K[q] \times K[q]$.

Seja $\varphi\left((q-\alpha) w_{1}\right)=\left(c_{0,0}, c_{0,1}\right)$ então $q-\alpha$ divide á $c_{0, i}$ e $\varphi\left(w_{1}\right)=\left(c_{1,0}, c_{1,1}\right)$ onde $(q-$ $\alpha) c_{1, i}=c_{0, i}$. Por outra lado, temos $\varphi\left((q-\alpha) w_{2}\right)=\left(1+\operatorname{Res}_{\alpha}(a)\right)\left(c_{1,0}, c_{1,1}\right)+\left(c_{2,0}, c_{2,1}\right), \mathrm{e}$ portanto existem $\beta_{2}, \mu_{2} \in K$ tal que $\beta_{2} c_{0,0}=\operatorname{Res}_{\alpha}(b) c_{2,0}$ e $\mu_{2} c_{0,1}=\operatorname{Res}_{\alpha}(a) c_{2,1}$. Então $(q-\alpha)^{2}$ divide á $c_{0, i}$, por indução existem $f_{0}, g_{0} \in K_{P o l\left(G_{1}\right)-\{\alpha\}}$ tal que

$$
\begin{aligned}
\varphi\left((q-\alpha) w_{1}\right) & =\left(\begin{array}{c}
\operatorname{Res}_{\alpha}(b)(q-\alpha)^{n} f_{0} \\
\operatorname{Res}_{\alpha}(a)(q-\alpha)^{n} g_{0}
\end{array}\right), \\
\varphi\left(w_{1}\right) & =\left(\begin{array}{c}
\operatorname{Res}_{\alpha}(b)(q-\alpha)^{n-1} f_{0} \\
\operatorname{Res}_{\alpha}(a)(q-\alpha)^{n-1} g_{0}
\end{array}\right), \\
\vdots & \\
\varphi\left(w_{i}\right) & =\left(\begin{array}{c}
d_{i, 1} \operatorname{Res}_{\alpha}(b)(q-\alpha)^{n-i} f_{0} \\
d_{i, 0} \operatorname{Res}_{\alpha}(a)(q-\alpha)^{n-i} g_{0}
\end{array}\right)+\sum_{j=0}^{i-1}\left(\begin{array}{c}
\beta_{i, j}(q-\alpha)^{n-j} f_{0} \\
\mu_{i, j}(q-\alpha)^{n-j} g_{0}
\end{array}\right),
\end{aligned}
$$

onde $d_{i, 0}=\left(i+\operatorname{Res}_{\alpha}(a)\right) d_{i-1,0}, d_{i, 1}=\left(i+\operatorname{Res}_{\alpha}(a)\right) d_{i-1,1}$ e $d_{0,0}=d_{0,1}=1$.

Do anterior $\varphi\left(\left(\begin{array}{l}1 \\ 0\end{array}\right)\right)=\left(\begin{array}{c}f_{0} \\ 0\end{array}\right)$ e $\varphi\left(\left(\begin{array}{l}0 \\ 1\end{array}\right)\right)=\left(\begin{array}{c}0 \\ g_{0}\end{array}\right)$ ou $(q-\alpha)^{j} w_{n}=w_{n-j}$.

No primeiro caso do homomorfismo $\varphi$ temos a ação de $A_{1}$ sobre $K_{P o l\left(G_{1}\right)-\{\alpha\}} \times K_{P o l\left(G_{1}\right)-\{\alpha\}}$ dada por; $p\left(\begin{array}{l}1 \\ 0\end{array}\right)=\left(\begin{array}{l}f_{0}^{-1} f_{0}^{\prime} \\ f_{0}^{-1} g_{0}\end{array}\right), p\left(\begin{array}{l}0 \\ 1\end{array}\right)=\left(\begin{array}{c}-b g_{0}^{-1} f_{0} \\ -a-g_{0}^{-1} g_{0}^{\prime}\end{array}\right)$. Então

$$
p\left(\begin{array}{l}
f \\
g
\end{array}\right)=\left(\begin{array}{c}
f^{\prime}+\left(f_{0}^{-1} f_{0}^{\prime}\right) f-g b g_{0}^{-1} f_{0} \\
g^{\prime}+\left(f_{0}^{-1} g_{0}\right) f-\left(a+g_{0}^{-1} g_{0}^{\prime}\right) g
\end{array}\right) .
$$

Portanto, $L$ é gerado por $\left(\begin{array}{c}f_{0} \\ g_{0}\end{array}\right)$ sobre $K[q]$ ou é gerado por $\left(\begin{array}{c}f_{0} \\ 0\end{array}\right),\left(\begin{array}{c}0 \\ g_{0}\end{array}\right)$ sobre $K[q]$. 
Baseados na observação anterior temos os seguintes lemas, os quais dão condições necessárias para determinar, quando um polo $\alpha \in \operatorname{Pol}\left(G_{1}\right)$ é essencial.

Lema 21. Seja $\alpha \in \Delta\left(G_{1}\right)$. Se $\operatorname{dim}_{K}(T(\alpha))$ é infinito enumerável então $\alpha$ é um polo essencial.

Demonstração. Se $\alpha$ não é polo essencial de $G_{1}=p^{2}+a p+b$, existe $F_{2}=p^{2}+g p+f \in \mathfrak{B}$ similar a $G_{1}$, tal que $\alpha \notin \operatorname{Pol}\left(F_{2}\right)$ e $G_{1}\left(x p+x_{1}\right)=\left(x p+x_{0}\right) F_{2}$, onde $\nu_{\alpha}\left(x_{1}\right), \nu_{\alpha}(x), \nu_{\alpha}\left(x_{0}\right)$ são não negativos, e $x_{1}, x$ não são nulos simultaneamente. Definimos o homomorfismo $\phi$ entre os $A_{1}$-módulos $W$ e $K_{P o l\left(G_{1}\right)-\{\alpha\}} \times K_{P o l\left(G_{1}\right)-\{\alpha\}}$ como segue:

$$
\phi\left(\left(\begin{array}{l}
1 \\
0
\end{array}\right)\right)=\left(\begin{array}{c}
x_{1} \\
x
\end{array}\right)
$$

é claro que $\phi$ está bem definido. Do homomorfismo $\phi$ segue

$$
\phi(K[q](T(\alpha))=L \subset K[q] \times K[q] .
$$

Por outra lado $\varphi$ induz um isomorfismo de $\operatorname{Soc}_{A_{1}}\left(\mathfrak{B} / \mathfrak{B} G_{1}\right)$ em $\operatorname{Soc}_{A_{1}}\left(\mathfrak{B} / \mathfrak{B} F_{2}\right)$, assim existe $m \in \mathbb{N}$ tal que $\varphi\left(\sum_{i=0}^{m} f_{i} p^{i}\left(\begin{array}{l}1 \\ 0\end{array}\right)\right)=\left(\begin{array}{l}1 \\ 0\end{array}\right)$ onde $f_{i}$ e $q-\alpha$ são relativamente primos. Multiplicando a última igualdade por $h_{\alpha}^{n+1}$ temos

$$
\varphi\left(\sum_{i=0}^{m} h_{\alpha}^{i-1} p^{i}\left(\begin{array}{l}
1 \\
0
\end{array}\right)\right)=h_{\alpha}^{n+1} u
$$

Usando $\left(h_{\alpha}^{n+3} F_{2} h_{\alpha}^{-n-1}\right)\left(h_{\alpha}^{n+1} u\right)=0$ temos que $\sum_{i=0}^{m+2} h_{\alpha}^{i-1} p^{i}\left(\begin{array}{l}1 \\ 0\end{array}\right)=0$. Portanto, $\operatorname{dim}_{K}(T(\alpha))=$ $n$, isto é absurdo. Logo $\alpha$ é um polo essencial.

Lema 22. Suponhamos que $\nu_{\alpha}(a)=-1$ e $\nu_{\alpha}(b) \geq-1$. Se $\alpha \in \Delta\left(G_{1}\right)$ e $\operatorname{dim}_{K}(T(\alpha))=n=$ $-\operatorname{Res}_{\alpha}(a)$, então a é essencial.

Demonstração. Da observação anterior, se $T(\alpha)$ não é gerado por $w_{n}$ então $\alpha$ é essencial. No outro caso $T(\alpha)$ é gerado por $w_{n}$ sobre $K[q]$, ver observação 5 . Assim $\varphi\left(\left(\begin{array}{l}1 \\ 0\end{array}\right)\right)=$ $\left(\begin{array}{c}(q-\alpha)^{n} \\ 0\end{array}\right)$, dado que $a \neq b$. No caso $a=b,|T(\alpha)|$ é infinito enumerável. Logo $\alpha$ é um polo essencial.

Seja $\bar{\Delta}\left(G_{1}\right)=\left\{\alpha \in \Delta\left(G_{1}\right) \mid \nu_{\alpha}(a)=-1, \nu_{\alpha}(b) \geq-\operatorname{Res}_{\alpha}(a) \in \mathbb{Z}\right\}$. Decorre dos lemas anteriores o seguinte corolário

Corolario 3. $\alpha \in \bar{\Delta}\left(G_{1}\right)$ se, e somente se, $\alpha$ não é essencial.

Definição 19. Dizemos que um polinômio $F \in \mathfrak{B}$ irredutivel é reduzido, se todos seus polos são essenciais. 
Do corolário anterior, existe $F_{1}$ similar a $G_{1}$ tal que $\bar{\Delta}\left(F_{1}\right)=\emptyset$, isto é, para $\alpha \in \operatorname{Pol}\left(F_{1}\right)$ e $\operatorname{Res}_{\alpha}\left(a_{1}\right) \in \mathbb{Z}$ temos $\nu_{\alpha}\left(a_{2}\right) \leq-\operatorname{Res}_{\alpha}(a)-1$. Dos lemas anterior o polinômio $F_{1}$ construido em (2.2) é reduzido, além disso a estrutura do $A_{1}$-módulo $W$ está determinada salvo isomorfismos pelo polonômio $F_{1}$.

Teorema 26. $\Delta\left(F_{1}\right) \neq \varnothing$ se, e somente se, $\left(\begin{array}{l}1 \\ 0\end{array}\right)$ não gera $K_{\operatorname{Pol}\left(F_{1}\right)} \times K_{P o l\left(F_{1}\right)}$.

Demonstração. $(\Rightarrow)$ Consequência da Lema (20).

$(\Leftarrow)$ Consequência do Lema (19).

Corolario 4. $K\left[q,\left(q-\alpha_{1}\right)^{-1}, \cdots,\left(q-\alpha_{l}\right)^{-1}\right] \times K\left[q,\left(q-\alpha_{1}\right)^{-1}, \cdots,\left(q-\alpha_{l}\right)^{-1}\right]$ é irredutivel, se é somente se, $\Delta\left(F_{1}\right)=\varnothing$

Corolario 5. $K\left[q,\left(q-\alpha_{1}\right)^{-1}, \cdots,\left(q-\alpha_{l}\right)^{-1}\right] \times K\left[q,\left(q-\alpha_{1}\right)^{-1}, \cdots,\left(q-\alpha_{l}\right)^{-1}\right]$ é cíclico gerado por $\left(\begin{array}{c}\prod_{\alpha \in \Delta\left(F_{1}\right)}(q-\alpha)^{\operatorname{Res}_{\alpha}(a)-2} \\ 0\end{array}\right)$.

Considere o $A_{1}$-módulo

$$
W^{\prime}=A_{1}\left(\begin{array}{c}
\prod_{\alpha \in \Delta\left(F_{1}\right)}(q-\alpha)^{-1} \\
0
\end{array}\right)
$$

Lema 23. Para $\alpha \in \Delta\left(F_{1}\right)$, os módulos $W_{\alpha}$ contem propriamente ao módulo $W$, além disso temos o isomorfismo

$$
\bigoplus_{\alpha \in \Delta\left(F_{1}\right)} M(\alpha) \simeq\left(K_{P o l\left(F_{1}\right)} \times K_{P o l\left(F_{1}\right)}\right) / W^{\prime}
$$

Demonstração. Denotaremos por $\bar{V}$ o módulo quociente

$$
\left(K_{P o l\left(F_{1}\right)} \times K_{P o l\left(F_{1}\right)}\right) / W^{\prime} .
$$

Como $K\left[q,(q-\alpha)^{-1}\right] \times K\left[q,(q-\alpha)^{-1}\right] \subset W_{\alpha}$, então para cada $w \in K_{P o l\left(F_{1}\right)} \times K_{P o l\left(F_{1}\right)}$, existe $m_{\alpha} \in \mathbb{N}$ tal que $h_{\alpha}^{m_{\alpha}} w \in W_{\alpha}$, portanto $w=\sum x_{\alpha} h_{\alpha}^{m_{\alpha}} w$, para $x_{\alpha} \in K[q]$ e $K_{P o l\left(F_{1}\right)} \times$ $K_{P o l\left(F_{1}\right)}=\sum W_{i}$.

Vejamos que $\bigcap_{\alpha \in \Delta\left(F_{1}\right)} W_{\alpha}=W^{\prime}$. Para $w \in \bigcap_{\beta \in \Delta\left(F_{1}\right)} W_{\beta}-W^{\prime}$, existe $f_{\beta} \in A_{1}$ tal que $f_{\beta} w=\left(\begin{array}{c}(q-\beta)^{-\theta_{\beta}\left(F_{1}\right)-3} \\ 0\end{array}\right) \in W_{\alpha}$, então $K\left[q,(q-\alpha)^{-1}\right] \times K\left[q,(q-\alpha)^{-1}\right] \subset W_{\alpha}$ para todo $\alpha \in \operatorname{Pol}\left(F_{1}\right)$, e portanto $W_{\alpha}=K_{P o l\left(F_{1}\right)} \times K_{P o l\left(F_{1}\right)}$, para cada $\alpha, \beta \in \Delta\left(F_{1}\right)$. Logo $W^{\prime}=K_{P o l\left(F_{1}\right)} \times K_{P o l\left(F_{1}\right)}$, o que é absurdo. Ver $(20)$.

Por outra parte, cada $\bar{W}_{\alpha}=W_{\alpha} / W^{\prime}$ é um $A_{1}$-módulo de $S$-torção irredutível. Fixando $\bar{w} \in \bar{W}_{\alpha}$ temos $h_{\alpha}^{d_{\alpha}} w \in K\left[q,(q-\alpha)^{-1}\right] \times K\left[q,(q-\alpha)^{-1}\right]$ para algum $d_{\alpha} \in \mathbb{N}$. Como $w \notin W^{\prime}$, existe $f \in A_{1}$ tal que $f w=\left(\begin{array}{c}(q-\alpha)^{-\theta_{\alpha}\left(F_{1}\right)-2} \\ 0\end{array}\right)$, assim $K\left[q,(q-\alpha)^{-1}\right] \times K\left[q,(q-\alpha)^{-1}\right] \subset W_{\alpha}$ e

$$
\bar{V}=\sum \bigoplus_{\alpha \in \Delta\left(F_{1}\right)} \bar{W}_{\alpha}
$$


Como $\bar{W}_{\text {alpha }}$ é irredutíveis de $S$-torção, então $\bar{W}_{\alpha} \simeq M(\alpha)$ e

$$
\bar{V}=\sum \bigoplus_{\alpha \in \Delta\left(F_{1}\right)} M(\alpha)
$$

Seja $\Delta_{0}\left(F_{1}\right) \subset \Delta\left(F_{1}\right)$ o conjunto definido por $\Delta_{0}\left(F_{1}\right)=\left\{\alpha \in \Delta\left(F_{1}\right) \mid \operatorname{dim}_{K}(T(\alpha))<\infty\{\right.$.

Afirmação 8. Temos o isomorfismo $\bigoplus_{\alpha \in \Delta_{0}\left(F_{1}\right)} M(\alpha) \simeq W^{\prime} / W$.

Demonstração. Segue-se da afirmação anterior.

Afirmação 9. Se $\alpha \in \Delta_{0}\left(F_{1}\right)$, então $W_{\alpha} / W \in \operatorname{Ext}^{1}(M(\alpha), M(\alpha))$.

Demonstração. Segue-se da afirmação anterior.

Para cada $\alpha \in \Delta\left(F_{1}\right)$ é definido o seguinte $A_{1}$-módulo

$$
V_{\alpha}=\left\{v \in \bar{V} \mid \exists m \in \mathbb{N} t q(q-\alpha)^{m} v=0\right\} .
$$

É evidente que $\bar{W}_{\alpha} \subset V_{\alpha}$. portanto $\left(K_{P o l\left(F_{1}\right)} \times K_{P o l\left(F_{1}\right)}\right) / W$ é suma dos módulos de peso $V_{\alpha}$.

Afirmação 10. Para os módulos $W$ e $W_{\alpha}$ temos

$$
\operatorname{Ext}_{A_{1}}^{1}\left(M(\alpha), W^{\prime}\right)=\left\{W^{\prime} \oplus M(\alpha), W_{\alpha}\right\} .
$$

Demonstração. Seja $V$ um $A_{1}$ módulo tal que a sequencia

$$
0 \longrightarrow W^{\prime} \stackrel{\varphi}{\longrightarrow} V \stackrel{\psi}{\longrightarrow} M(\alpha) \longrightarrow 0
$$

é exata. Se $V$ é livre de $S$-torção, então $V=A v$ onde $v \notin \operatorname{Im}(\varphi)$ e $(q-\alpha) v \in \operatorname{Im}(\varphi)$. Dado que $F_{1} n=0$, então $F_{1} f(q-\alpha) v=0$ para algum $f \in A_{1}$. Como $\mathfrak{B}$ é um D.I.P. temos $F_{1} f=g \gamma_{v}$ onde $\gamma_{v}$ é o polinômio irredutível anulador de $(q-\alpha) v$. Portanto, $F_{1}$ e $\gamma_{v}$ são similares, assim a única classe salvo isomorfismos no $\operatorname{Ext}_{A_{1}}^{1}\left(M(\alpha), W^{\prime}\right)$, é $W_{\alpha}$.

Afirmação 11. Para os módulos $W, W^{\prime}$ temos $\operatorname{Ext}_{A_{1}}^{1}(W, M(\alpha))=\left\{W \oplus M(\alpha), W^{\prime}\right\}$.

Demonstração. Segue-se da afirmação anterior.

Lema 24. $K\left[q,\left(q-\alpha_{1}\right)^{-1}, \cdots,\left(q-\alpha_{l}\right)^{-1}\right] \times K\left[q,\left(q-\alpha_{1}\right)^{-1}, \cdots,\left(q-\alpha_{l}\right)^{-1}\right]$ é de comprimento $\left|\Delta\left(F_{1}\right)\right|+\left|\Delta_{0}\left(F_{1}\right)\right|$, como $A_{1}$-módulo.

Demonstração. Usando os isomorfismos

$$
\begin{aligned}
W_{1 \cdots j} & \simeq W_{\alpha_{j}}+W_{1 \cdots(j-1)}, \\
W_{\alpha_{j}} \cap W_{1 \cdots(j-1)} & =W,
\end{aligned}
$$

temos $W_{1 \cdots j} / W_{1 \cdots(j-1)} \simeq W_{\alpha_{j}} / W$. Do anterior $W_{\alpha_{j}} / W$ é irredutivel o existe $W \subset V_{\alpha_{j}} \subset$ $W_{\alpha_{j}}$ tal que $W_{\alpha_{j}} / V_{\alpha_{j}}$ e $V_{\alpha_{j}} / W$ são irredutíveis. Portanto, $K_{P o l\left(F_{1}\right)} \times K_{P o l\left(F_{1}\right)}$ é de comprimento finito com longitude $\left|\Delta\left(F_{1}\right)\right|+\left|\Delta_{0}\left(F_{1}\right)\right|$. 
Afirmação 12. Para os módulos $W^{\prime}, K_{P o l\left(F_{1}\right)} \times K_{P o l\left(F_{1}\right)}$ temos

$$
\operatorname{Ext}_{A_{1}}^{1}\left(W^{\prime}, \bigoplus M\left(\alpha_{i}\right)\right)=\left\{W^{\prime} \bigoplus_{\alpha \in \Delta\left(F_{1}\right)} M(\alpha), K_{P o l\left(F_{1}\right)} \times K_{P o l\left(F_{1}\right)}\right\}
$$

Demonstração. $\operatorname{Ext}_{A_{1}}^{1}\left(W^{\prime}, \bigoplus M\left(\alpha_{i}\right)\right) \simeq \bigoplus \operatorname{Ext}_{A_{1}}^{1}\left(W^{\prime}, M\left(\alpha_{i}\right)\right)$.

Para $\alpha \in \Delta\left(F_{1}\right)$, do anterior temos a seguinte sequência exata de $A_{1}$-módulos.

$$
0 \longrightarrow W \stackrel{\varphi}{\longrightarrow} K_{\text {Pol }\left(F_{1}\right)} \times K_{P o l\left(F_{1}\right)} \stackrel{\psi}{\longrightarrow}\left(K_{P o l\left(F_{1}\right)} \times K_{P o l\left(F_{1}\right)}\right) / W \simeq W \bigoplus M\left(\alpha_{i}\right) \longrightarrow 0
$$




\section{Capítulo 3}

\section{Módulos irredutíveis de grau dois sobre $S l_{2}(K)$}

Nesta seção damos uma caracterização do $\operatorname{Soc}_{\rho_{\lambda}\left(U_{s}\right)}(N)$, para $N$ um $\mathfrak{B}$-módulo irredutível isomorfo a $\mathfrak{B} / \mathfrak{B} F_{1}$. Além disso, descrevemos a relação que existe entre os $U s$-módulos $W$ e $S_{o c} c_{\rho_{\lambda}\left(U_{s}\right)}(N)$. Fixando $\lambda \in K$, temos a relação $Q_{0, F_{1}}(\xi-\lambda)=Q_{0, F_{1, \lambda}}(\xi)$, onde $F_{1, \lambda}=$ $F_{1}\left(p-\lambda q^{-1}, q\right)$ ou equivalentemente $F_{1, \lambda}=q^{\lambda} F_{1} q^{-\lambda}$. Seja $Q_{0, F_{1, \lambda}}(\xi)$ o polinômio principal associado a $F_{1, \lambda}$.

$F_{1, \lambda}$ não é preservado se, e somente se, existe $n \in \mathbb{N}$ tal que $-\lambda-n=\theta_{i}$, onde $\theta_{1}, \theta_{2}$ são as raízes do polinômio $Q_{0, F_{1}}(\xi)$. Definimos $D^{+}\left(D^{-}\right)=\left\{-\theta_{1},-\theta_{2}\right\}+\mathbb{Z}^{+}\left(\mathbb{Z}^{-}\right)$e $D=D^{+} \cup D^{-}$. Nesta seção temos os seguintes resultados

Lema 25. Suponhamos que $0 \notin \operatorname{Pol}\left(F_{1}\right)$ então $\operatorname{Soc}_{\rho_{\lambda}\left(U_{s}\right)}(N)=W$ salvo quando $\lambda \in \mathbb{Z}^{-}$. Se $\lambda \in \mathbb{Z}^{-}$temos $\operatorname{Soc}_{\rho_{\lambda}\left(U_{s}\right)}(N)=q^{-\lambda} W$.

Lema 26. Sejam $\alpha_{1}=0 \in \operatorname{Pol}\left(F_{1}\right)$ e $\theta_{1}, \theta_{2}$ as raizes do polinômio principal $Q_{0, F_{1}}(\xi)$ associado a $F_{1}$. Se $\lambda \notin D$ o polinômio $\rho_{\lambda} \rho_{\lambda}^{-1}\left(F_{1}\right)$ resulta preservado e $\operatorname{Soc}_{\rho_{\lambda}\left(U_{s}\right)}(N)=W$.

Afirmação 13. Sejam $\lambda \in K e V_{\sigma}=q^{t} W / \operatorname{Soc}_{\rho_{\lambda}\left(U_{s}\right)}(N)\left(W / S o c_{\rho_{\lambda}\left(U_{s}\right)}(N)\right)$. Então $V_{\sigma} \quad e ́$ um módulo de Verma com peso $\sigma$, onde $\sigma$ toma um dos seguintes valores

1. $-\lambda-1$

2. $-\lambda-t$ se $-\lambda-t$ é raiz de $Q_{0, F_{1}}(\xi)$ para $r_{1}=s_{1}-1$.

3. $-\lambda+t$ se $-\lambda+t$ é raiz de $Q_{0, F_{1}}(\xi)$ para $r_{1}=s_{1}-1$.

O resultado principal do trabalho, é enunciado no final desta seção.

Doravante todos os módulos considerados são módulo sobre $\rho(\lambda) U s$.

Lembremos que $F_{1}=p^{2}+a p+b$ onde

$$
a=\frac{a_{1}}{\prod\left(q-\alpha_{i}\right)^{r_{i}}}, b=\frac{b_{1}}{\prod\left(q-\alpha_{i}\right)^{s_{i}}} .
$$




\subsubsection{Se $0 \notin \operatorname{Pol}\left(F_{1}\right)$}

Sejam $g=\prod\left(q-\alpha_{i}\right)^{l_{i}}, g_{i}=g\left(q-\alpha_{i}\right)^{-l_{i}}$, onde $l_{j}=\max \left\{r_{j}, s_{j}\right\}$. Lembremos que $e=q, f=$ $-(q p+\lambda+1) p, h=2 q p+\lambda+1, u=\left(\begin{array}{l}1 \\ 0\end{array}\right)$ e $p u=\left(\begin{array}{l}0 \\ 1\end{array}\right)$.

Seja $M$ um $U_{s}$-submódulo de $\rho(\lambda) U s\left(\begin{array}{l}1 \\ 0\end{array}\right)$. Como $K[q] \subset \rho(\lambda) U s$ existem $f_{1}, f_{2} \in K[q]$ tal que $v=f_{1} u+f_{2} p u \in M-\{0\}$. Da ação de $h$ sobre o elemento $v$ temos $g q p \in M$, onde

$$
g q p v=\left(\begin{array}{c}
g q f_{1}^{\prime}-q g b f_{2} \\
q g f_{1}+q g f_{2}^{\prime}-g a q f_{2}
\end{array}\right) \in M
$$

então $\left(f_{1} g q p-\left(g q f_{1}^{\prime}-q g b f_{2}\right)\right) v=x u$ e $\left(f_{2} g q p-\left(q g f_{1}+q g f_{2}^{\prime}-g a q f_{2}\right)\right) v=-x p u$, para $0 \neq x=q g f_{1}^{\prime} f_{2}-q g b f_{2}^{2}-q g f_{1}^{2}-q g f_{2}^{\prime} f_{1}+q g a f_{2} f_{1}$.

Do anterior, existe $y \in K[q]$ tal que $u_{1}=\left(\begin{array}{l}y \\ 0\end{array}\right), u_{2}=\left(\begin{array}{l}0 \\ y\end{array}\right) \in M$. Usando indução e a ação de $h$ nos elementos anteriores, existem $n, m_{i} \in \mathbb{N}$ para $i=1, \cdots, n$ tais que

$$
d_{0}=\left(\begin{array}{c}
q^{n} \prod\left(q-\alpha_{i}\right)^{m_{i}} \\
0
\end{array}\right), d_{1}=\left(\begin{array}{c}
0 \\
q^{n} \prod\left(q-\alpha_{i}\right)^{m_{i}}
\end{array}\right) \in M .
$$

No que segue daremos uma lista de elementos de $M$ obtidos da ação de $h$, os quais denotaremos por $d_{2}, d_{3}, d_{4}$ e $d_{5}$. Os elementos $d_{2}$ e $d_{3}$ e $d_{4}$ são obtidos da ação de $h$ sobre os elementos $d_{0}, d_{1}$ e $d_{2}$ respectivamente. Finalmente $d_{5}$ é obtido de $g_{k} d_{3}$.

$$
\begin{aligned}
& d_{2}=q^{n} \prod_{i}\left(q-\alpha_{i}\right)^{m_{i}-1} u \\
& d_{3}=\left(\begin{array}{c}
-b q^{n+1}\left(q-\alpha_{i}\right)^{m_{i}} \prod_{j \neq i}\left(q-\alpha_{j}\right)^{m_{j}} \\
\sum_{i} m_{i} q^{n+1}\left(q-\alpha_{i}\right)^{m_{i}-1} \prod_{j \neq i}\left(q-\alpha_{j}\right)^{m_{j}}-a q^{n+1} \prod\left(q-\alpha_{j}\right)^{m_{j}}
\end{array}\right) . \\
& d_{4}=\left(\begin{array}{c}
\left(m_{i}-1\right) q^{n+1}\left(q-\alpha_{i}\right)^{m_{i}-2} \prod_{j \neq i}\left(q-\alpha_{j}\right)^{m_{j}} \\
q^{n+1}\left(q-\alpha_{i}\right)^{m_{i}-1} \prod_{j \neq i}\left(q-\alpha_{j}\right)^{m_{j}}
\end{array}\right) . \\
& d_{5}=\left(\begin{array}{c}
-b g_{k} q^{n+1}\left(q-\alpha_{k}\right)^{m_{k}} \prod_{j \neq k}\left(q-\alpha_{j}\right)^{m_{j}} \\
m_{k} g_{k} q^{n+1}\left(q-\alpha_{k}\right)^{m_{k}-1} \prod_{j \neq k}\left(q-\alpha_{j}\right)^{m_{j}}-a g_{k} q^{n+1} \prod\left(q-\alpha_{j}\right)^{m_{j}}
\end{array}\right) .
\end{aligned}
$$

Exemplo 5. Suponhamos que $\operatorname{Pol}\left(F_{1}\right)=\left\{\alpha_{1}, \alpha_{2}\right\}, r_{1}=0$ e $s_{1}=1$. Seja $l_{2}=\max \left\{\nu_{\alpha_{2}}(a), \nu_{\alpha_{2}}(b)\right\}$, então de $\left(q-\alpha_{2}\right)^{l_{2}} q p d_{1}$ temos

$$
c_{0}=\left(\begin{array}{c}
0 \\
q^{n}\left(q-\alpha_{1}\right)^{m_{1}-1}\left(q-\alpha_{2}\right)^{m_{2}+l_{2}}
\end{array}\right) \in M .
$$

Usando o elemento $d_{1}$ e $c_{0}$ obtemos $\left(q-\alpha_{1}\right) d_{1} \in M$. Usando indução sobre $m_{1}-1$ obtemos

$$
\left(\begin{array}{c}
0 \\
q^{n}\left(q-\alpha_{2}\right)^{m_{2}}
\end{array}\right) \in M
$$


Trocando $\alpha_{1}$ por $\alpha_{2}$ no argumento anterior, mostramos que $\left(\begin{array}{c}0 \\ q^{n}\left(q-\alpha_{1}\right)^{m_{1}}\end{array}\right) \in M$. Portanto existe $n \in \mathbb{N}$ tal que

$$
\left(\begin{array}{c}
0 \\
q^{n}
\end{array}\right),\left(\begin{array}{c}
q^{n} \\
0
\end{array}\right) \in M
$$

O exemplo acima dá uma motivação para mostrar que em todo submódulo $M$ de $\rho(\lambda) U s$ existem elementos $\left(\begin{array}{c}0 \\ q^{n}\end{array}\right),\left(\begin{array}{c}q^{n} \\ 0\end{array}\right)$ para algum $n \in \mathbb{N}$. Analogamente ao exemplo, considere os seguintes elementos de $M$, gerados por $q p d_{2}$ e $d_{1}$. De $q p d_{2}$ temos o elemento

$$
v_{0}=q^{n} \prod_{j}\left(q-\alpha_{j}\right)^{m_{j}-\delta_{i j}} p u+\left(m_{i}-1\right) q^{n} \prod_{j}\left(q-\alpha_{j}\right)^{m_{j}-2 \delta_{i j}} u \in M
$$

De $f \prod_{j \neq i}\left(q-\alpha_{j}\right) d_{1}$ temos o elemento

$$
(q p+\lambda+1)\left[q^{n-1} \prod_{j}\left(q-\alpha_{j}\right)^{m_{j}-\delta_{i j}}\left[\left(n+\left(m_{i}-1\right) q\left(q-\alpha_{i}\right)^{-1}\right) u+q p u\right]\right] \in M
$$

Então $(q p+\lambda+1)\left(q^{n-1} \prod_{j}\left(q-\alpha_{j}\right)^{m_{j}-\delta_{i j}}\right) u=(n+\lambda)\left(q^{n-1} \prod_{j}\left(q-\alpha_{j}\right)^{m_{j}-\delta_{i j}} u\right)+v_{0}$, dado que $v_{0} \in M$ tem-se

$$
q^{n-1} \prod_{j}\left(q-\alpha_{j}\right)^{m_{j}-\delta_{i j}} u \in M
$$

Se $\lambda \notin \mathbb{Z}^{-}, \operatorname{Soc}_{\rho_{\lambda}\left(U_{s}\right)}(N)$ é irredutível. Para nosso caso $F_{1, \lambda}=\rho_{-\lambda} \rho_{\lambda}^{-1}\left(F_{1}\right)$ é preservado se, e somente se, $\lambda \notin \mathbb{Z}^{-}$, já que $Q_{0, F_{1, \lambda}}(\xi)=(\xi-\lambda)(\xi-1-\lambda)$.

Afirmação 14. Para todo $\rho(\lambda) U s$-submódulo $M$ de $\rho(\lambda) U s\left(\begin{array}{l}1 \\ 0\end{array}\right)$, existe $n \in \mathbb{N}$ tal que $\left(\begin{array}{c}q^{n} \\ 0\end{array}\right),\left(\begin{array}{c}0 \\ q^{n}\end{array}\right) \in M$

Demonstração. Sejam $M$ um submódulo de $\rho(\lambda) U s\left(\begin{array}{l}1 \\ 0\end{array}\right)$ e $m=\left(m_{1}, \cdots, m_{l}\right)$ com $|m|=$ $\sum m_{i}$ mínima tal que $q^{n} \prod_{j}\left(q-\alpha_{j}\right)^{m_{j}} p u, d_{1}=q^{n} \prod\left(q-\alpha_{j}\right)^{m_{j}-1} u \in M$.

1. Suponhamos que $r_{i}=0$ e $s_{i}=1$, então $Q_{\alpha_{i}, F_{1}}(\xi)=\xi(\xi-1)$. De $d_{5}$ e usando (3.1) temos

$$
\left(\begin{array}{c}
-R e s_{\alpha_{k}}(b) q^{n+1}\left(q-\alpha_{k}\right)^{m_{k}-1} \prod_{j \neq k}\left(q-\alpha_{j}\right)^{m_{j}} \\
m_{k} q^{n+1}\left(q-\alpha_{k}\right)^{m_{k}-1} \prod_{j \neq k}\left(q-\alpha_{j}\right)^{m_{j}}
\end{array}\right) \in M
$$

então $q^{n+1} \prod_{j}\left(q-\alpha_{j}\right)^{m_{j}-\delta_{i j}} p u \in M$, para $i=1, \cdots, l$. Do fato $q^{n} \prod_{j}\left(q-\alpha_{j}\right)^{m_{j}} p u \in M$ implica $q^{n} \prod_{j}\left(q-\alpha_{j}\right)^{m_{j}-1} p u \in M$.

2. Suponhamos que $r_{i}=1$ e $s_{i} \leq 1$, então $Q_{\alpha_{i}, F_{1}}(\xi)=\xi\left(\xi-1+\operatorname{Res}_{\alpha_{i}}(a)\right)$, onde $F_{1}$ não é preservado, se somente se, $\operatorname{Res}_{\alpha_{i}}(a) \geq 1$. De $d_{5}$ e usando (3.1) segue

$$
\left(\begin{array}{c}
0 \\
\left(m_{k}-\operatorname{Res}_{\alpha_{k}}(a)\right) q^{n+1}\left(q-\alpha_{k}\right)^{m_{k}-1} \prod_{j \neq k}\left(q-\alpha_{j}\right)^{m_{j}}
\end{array}\right) \in M .
$$

Portanto, $q^{n+1} \prod_{j}\left(q-\alpha_{j}\right)^{m_{j}-\delta_{i j}}\left(m_{i}-\operatorname{Res}_{\alpha_{i}}(a)\right) p u \in M$, para $i=1, \cdots, l$. Dado que $q^{n} \prod_{j}\left(q-\alpha_{j}\right)^{m_{j}} p u \in M$ temos $q^{n} \prod_{j}\left(q-\alpha_{j}\right)^{m_{j}-1} p u \in M$. 
3. Suponhamos que $r_{i} \geq 0$ e $s_{i}=2$, então $Q_{\alpha_{i}, F_{1}}(\xi)=\xi(\xi-1)+R e s_{\alpha}(b)$. De $d_{5}$ e usando (3.1) obtemos

$$
\left(\begin{array}{c}
\operatorname{Res}_{\alpha_{k}}(b) q^{n+1}\left(q-\alpha_{k}\right)^{m_{k}-2} \prod_{j \neq k}\left(q-\alpha_{j}\right)^{m_{j}} \\
m_{k} q^{n+1}\left(q-\alpha_{k}\right)^{m_{k}-1} \prod_{j \neq k}\left(q-\alpha_{j}\right)^{m_{j}}
\end{array}\right) \in M
$$

Do elemento anterior e de $d_{4}$ segue-se $\left(m_{i}\left(m_{i}+1\right)-\operatorname{Res}_{\alpha_{i}}(b)\right) q^{n+1} \prod_{j}\left(q-\alpha_{j}\right)^{m_{j}-\delta_{i j}} p u \in$ $M$, para $i=1, \cdots, l$. Como $F_{1}$ é preservado temos $q^{n} \prod_{j}\left(q-\alpha_{j}\right)^{m_{j}-1} p u \in M$.

4. Suponhamos que $r_{i}=1$ e $s_{i}=2$, então $Q_{\alpha_{i}, F_{1}}(\xi)=\xi(\xi-1)+\operatorname{Res}_{\alpha}(a) \xi+\operatorname{Res}_{\alpha}(b)$. De $d_{5}$ e usando (3.1) temos

$$
\left(\begin{array}{c}
\operatorname{Res}_{\alpha_{k}}(b) q^{n+1}\left(q-\alpha_{k}\right)^{m_{k}-2} \prod_{j \neq k}\left(q-\alpha_{j}\right)^{m_{j}} \\
\left(m_{k}-\operatorname{Res}_{\alpha_{k}}(a)\right) q^{n+1}\left(q-\alpha_{k}\right)^{m_{k}-1} \prod_{j \neq k}\left(q-\alpha_{j}\right)^{m_{j}}
\end{array}\right) \in M .
$$

Do elemento anterior e de $d_{4}$ segue-se $\left(m_{i}\left(m_{i}+1-\operatorname{Res}_{\alpha}(a)\right)-\operatorname{Res}_{\alpha_{i}}(b)\right) q^{n+1} \prod_{j}(q-$ $\left.\alpha_{j}\right)^{m_{j}-\delta_{i j}} p u \in M$, para $i=1, \cdots, l$. Como $F_{1}$ é preservado temos $q^{n} \prod_{j}\left(q-\alpha_{j}\right)^{m_{j}-1} p u \in$ $M$.

5. A demonstração nos casos restantes, é similar.

Assim para $i=1, \cdots, n$,

$$
Q_{\alpha_{i}, F_{1}}\left(-m_{i}\right) q^{n} \prod_{j \neq i}(q-\alpha)^{m_{j}-\delta_{i j}} \in M
$$

Portanto, $\prod_{j \neq i}(q-\alpha)^{m_{j}-1} \in M$. Segue-se da hipótese que $q^{n} u, q^{n} p u \in M$ para algum $n \in \mathbb{N}$.

Note que na demonstração da afirmação 14, não foi usado o fato que $\alpha_{i} \neq 0$ para $i=$ $1, \cdots, n$.

Lema 27. Suponhamos que $0 \notin \operatorname{Pol}\left(F_{1}\right)$ então $\operatorname{Soc}_{\rho_{\lambda}\left(U_{s}\right)}(N)=W$ salvo quando $\lambda \in \mathbb{Z}^{-}$. Se $\lambda \in \mathbb{Z}^{-}$temos $\operatorname{Soc}_{\rho_{\lambda}\left(U_{s}\right)}(N)=q^{-\lambda} W$.

Demonstração. Sejam $M$ um $\rho(\lambda) U s$-submódulo de $\rho(\lambda) U s\left(\begin{array}{l}1 \\ 0\end{array}\right)$ e $m$ o menor inteiro positivo tal que $q^{m} u, q^{m} p u \in M$. Note que $h q^{n} u, h q^{n} p u$ são múltiplos de $q^{n} u, q^{n} p u$ sobre $K[q]$. De fup se segue

$$
\begin{aligned}
(q p+\lambda+1) p\left(q^{m} p u\right) & =(q p+\lambda+1)\left(q^{m} p^{2} u+m q^{m-1} p u\right), \\
& =q^{m+1} p^{3} u+(2 m+\lambda+1) q^{m} p^{2} u+m(\lambda+m) q^{m-1} p u . \\
(q p+\lambda+1) p\left(q^{m} u\right) & =m q^{m} p^{u}+m(m+\lambda) q^{m-1} u .
\end{aligned}
$$

Multiplicando a equação (3.3) por $g^{4}$, segue-se que $q^{m-1} u, q^{m-1} p u \in M$, para $-\lambda \neq m$. Dado que $m$ foi tomado como o menor inteiro positivo, então $\rho(\lambda) U s\left(\begin{array}{l}1 \\ 0\end{array}\right)$ é um $\rho_{\lambda} U_{s}$-módulo irredutível de $W$. 
Para provar que $\rho(\lambda) U s\left(\begin{array}{l}1 \\ 0\end{array}\right)=W$ basta mostrar que $p^{t} u \in \rho(\lambda) U s\left(\begin{array}{l}1 \\ 0\end{array}\right)$, para tudo $t \in \mathbb{N}$. Para $t=0$ a afirmação é válida. Suponhamos que a afirmação é válida para $t \leq k$. Defina $z_{t}=p^{t} u \in \rho(\lambda) U s\left(\begin{array}{l}1 \\ 0\end{array}\right)$, lembremos que $g \in K[q]$, é o polinômio de menor grau tal que $g a, g b \in K[q]$. Considerando o elemento $q p^{n+1} u=q z_{k+1}$, multiplicando por $g^{k+1}$ à esquerda o seguinte elemento

$$
f z_{k}=q p^{k+2} u+(\lambda+1) p^{k+1} u=q p^{k}(-b u-a p u)+(\lambda+1) p^{k+1} u \in \rho(\lambda) U s\left(\begin{array}{l}
1 \\
0
\end{array}\right),
$$

e usando a equação $q^{m} p^{j}=\sum_{i=0}^{j} \lambda_{i j} p^{j-i} D^{(i)}\left(q^{m}\right)$, obtemos $z_{k+1} \in \rho(\lambda) U s\left(\begin{array}{l}1 \\ 0\end{array}\right)$. Portanto $\rho(\lambda) U s\left(\begin{array}{l}1 \\ 0\end{array}\right)=A_{1}\left(\begin{array}{l}1 \\ 0\end{array}\right)$.

Se $\lambda \in \mathbb{Z}^{-}$, da afirmação anterior temos $q^{-\lambda} u, q^{-\lambda} p u \in M$, para todo $\rho(\lambda) U s$-submódulo irredutivel $M$ de $\rho(\lambda) U s\left(\begin{array}{l}1 \\ 0\end{array}\right)$, portanto $M=\rho(\lambda) U s\left(\begin{array}{c}q^{-\lambda} \\ 0\end{array}\right)$, é irredutível. Seja $k$ o maior inteiro positivo tal que $q^{-\lambda} p^{k} u \in M$. Substituindo $m$ por $-\lambda$ e $p$ por $p^{k}$ em (3.3), se segue que $q^{-\lambda} p^{k+1} u \in M$, além disso $q^{-\lambda} W$ é fechado sobre a ação de $f$.

\subsubsection{Se $0 \in \operatorname{Pol}\left(F_{1}\right)$}

Daremos uma motivação para nosso estudo, e ilustraremos o que acontece com o quociente $W / \operatorname{Soc}_{\rho_{\lambda}\left(U_{s}\right)}(N)$ para o caso $0 \in \operatorname{Pol}\left(F_{1}\right)$.

Exemplo 6. Suponhamos que $r_{1}=0, s_{1}=1$ e $\operatorname{Pol}\left(F_{1}\right)=\{0, \alpha\}$, então $F_{1}=p^{2}+a p+b q^{-1}$ onde $a, b \in K\left[q,(q-\alpha)^{-1}\right]$.

Para cada $\rho(\lambda) U$ s-submódulo $M$ de $W$, existe $n \in \mathbb{N}$ tal que $q^{n} u, q^{n} p u \in M$. Da ação de $f$ sobre os elementos $q^{n} u, q^{n} p u$, obtemos os seguintes elementos de $M$,

$$
(q p+\lambda+1)\left(q^{n-1} p u\right),(q p+\lambda+1)\left(q^{n-1} u\right),
$$

portanto $(\lambda+n) q^{n-1} p u,(\lambda+n) q^{n-1} u \in M$.

Se $\lambda \in \mathbb{Z}^{-}$, então $q^{-\lambda} u, q^{-\lambda} p u \in M$, além disso $q^{-\lambda} W$ obtém estrutura de $\rho(\lambda) U s$-módulo.

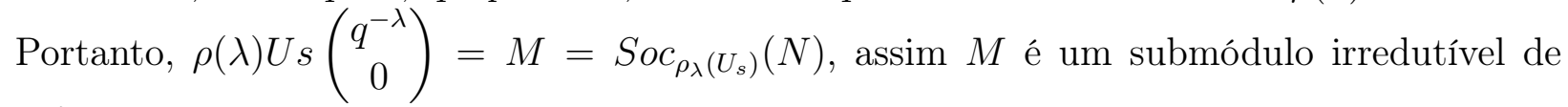
$q^{-\lambda} W$

O quociente $q^{-\lambda} W / S o c_{\rho_{\lambda}\left(U_{s}\right)}(N)$ dá informação da relação que existe entre os módulos

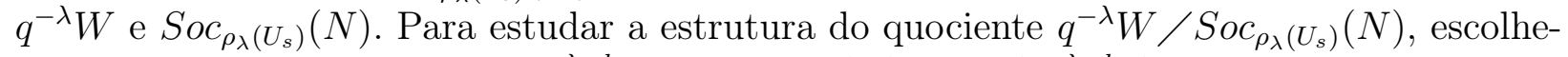
mos $k$ o maior inteiro tal que $q^{-\lambda} p^{k} u \in \operatorname{Soc}_{\rho_{\lambda}\left(U_{s}\right)}(N)$. De $f\left(q^{-\lambda} p^{k} u\right)$ temos

$$
(\lambda+k-1) q^{-\lambda} p^{k+1} u \in \operatorname{Soc}_{\rho_{\lambda}\left(U_{s}\right)}(N),
$$

e portanto o maior inteiro que satisfaz $q^{-\lambda} p^{k} u$ é $k=-\lambda+1$. Como $q^{-\lambda} p^{-\lambda+2} u \notin S o \rho_{\rho_{\lambda}\left(U_{s}\right)}(N)$. 
Definimos

$$
\begin{aligned}
& v_{0}=q^{-\lambda} p^{-\lambda+2} u \quad\left(\bmod \operatorname{Soc}_{\rho_{\lambda}\left(U_{s}\right)}(N)\right), \\
& v_{i}=f v_{i-1}, \quad i \geq 1 \quad\left(\bmod \operatorname{Soc}_{\rho_{\lambda}\left(U_{s}\right)}(N)\right) .
\end{aligned}
$$

O conjunto $\left\{v_{i} \mid i \in \mathbb{N}\right\}$ gera o módulo quociente $W / \operatorname{Soc}_{\rho_{\lambda}\left(U_{s}\right)}(N)$, neste módulo quociente temos $q v_{0}=0$.

$$
\begin{aligned}
h v_{0}=(2 q p+\lambda+1) v_{0} & =2 q^{-\lambda+1} p^{-\lambda+3} u+(-\lambda+1) q^{-\lambda} p^{-\lambda+2} u, \\
& =2 q^{-\lambda} p^{-\lambda+1}\left(q p^{2} u\right)-(-\lambda+1) q^{-\lambda} p^{-\lambda+2} u, \\
& \equiv(\lambda-1) q^{-\lambda} p^{-\lambda+2} u \quad\left(\bmod \operatorname{Soc}_{\rho_{\lambda}\left(U_{s}\right)}(N)\right) .
\end{aligned}
$$

Logo, $W / \operatorname{Soc}_{\rho_{\lambda}\left(U_{s}\right)}(N) \simeq V(\lambda-1)$ é um módulo de Verma de peso $\lambda-1$.

Se $\lambda \notin \mathbb{Z}^{-}$então $S_{o c_{\rho_{\lambda}}\left(U_{s}\right)}(N)=\rho(\lambda) U s\left(\begin{array}{l}1 \\ 0\end{array}\right)$. Por outro lado o módulo quociente

$$
W / \operatorname{Soc}_{\rho_{\lambda}\left(U_{s}\right)}(N)=W / \rho(\lambda) U s\left(\begin{array}{l}
1 \\
0
\end{array}\right),
$$

então $u, p u \in S_{o c} c_{\rho_{\lambda}\left(U_{s}\right)}(N)=\rho(\lambda) U s\left(\begin{array}{l}1 \\ 0\end{array}\right)$. Seja $k$ o maior inteiro tal que $p^{k} u \in \rho(\lambda) U s\left(\begin{array}{l}1 \\ 0\end{array}\right)$, de $f p^{k} u$ temos $q p^{k+2} u+(\lambda+1) p^{k+1} u \in M$. Dos seguintes elementos

$$
\begin{aligned}
q p^{k+2} u & =p^{k}\left(q p^{2}\right)-k p^{k+1} u, \\
& \equiv-k p^{k+1} u \quad(\bmod M),
\end{aligned}
$$

obtemos $(\lambda-k+1) p^{k+1} u \in M$, então $M=\operatorname{Soc}_{\rho_{\lambda}\left(U_{s}\right)}(N)=W$ para $\lambda \notin \mathbb{N}$. Suponhamos que $\lambda \in \mathbb{N}$, definindo

$$
\begin{aligned}
& v_{0}=p^{\lambda} u \\
& v_{i}=f v_{i-1}, \quad i \geq 1 \quad(\bmod M),
\end{aligned}
$$

o conjunto $\left\{v_{i} \mid i \in \mathbb{N}\right\}$ gera o módulo quociente $W / M$. De $f p^{\lambda-1}$ temos $q p^{\lambda+1} \equiv-(\lambda+$ 1) $p^{\lambda}(\bmod M) \mathrm{e}$

$$
\begin{aligned}
h v_{0}=(2 q p+\lambda+1) v_{0} & =2 q p^{\lambda+1} u+(\lambda+1) p^{\lambda} u \\
& \equiv-(\lambda+1) p^{\lambda+1} u \quad(\bmod M) .
\end{aligned}
$$

Como $q v_{0}=0$ temos $W / M \simeq V(-\lambda-1)$, onde $V(-\lambda-1)$ é o modulo de Verma de peso $-\lambda-1$. No exemplo anterior, é muito importante o fato que 0 é um polo de ordem um.

\subsubsection{Estrutura do $S o c_{\rho_{\lambda}\left(U_{s}\right)}(N)$}

Nesta seção generalizamos o exemplo anterior. A demonstração depende da forma do polinômio principal $Q_{\alpha_{i}, F_{1}}(\xi)$ associado a $F_{1}$. Portanto, a demonstração é dividida em 7 caos, como em (2.1).

Das propriedades obtidas na seção anterior, para todo $\rho(\lambda) U s$-módulo $M$ existe $n \in \mathbb{N}$ tal 
que $q^{n} u, q^{n} p u \in M$. Mostraremos que

$$
Q_{0, F_{1}}(-\lambda-n) q^{n-1} u, \quad Q_{0, F_{1}}(-\lambda-n) q^{n-1} p u \in M
$$

implicando $S_{o c} c_{\rho_{\lambda}\left(U_{s}\right)}(N)=\rho(\lambda) U s\left(\begin{array}{l}1 \\ 0\end{array}\right)$, sempre que $\lambda \notin D^{-}$. Por outra parte mostraremos que

$$
Q_{0, F_{1}}(-\lambda+n) p^{n+1} u \in M
$$

Portanto, $\operatorname{Soc}_{\rho_{\lambda}\left(U_{s}\right)}(N)=W$ para $\lambda \in K-D$.

Os elementos dados a continuação serão usados na demonstração das seguinte afirmação, e como consequência desta afirmação obtemos (3.6) e (3.7).

$$
\begin{aligned}
f\left(q^{m} p^{k} u\right) & =q^{m+1} p^{k+2} u+(2 m+\lambda+1) q^{m} p^{k+1} u+m(m+\lambda) q^{m-1} p^{k} u, \\
q p\left(q^{m} p^{k} u\right) & =q^{m+1} p^{k+1} u+m q^{m} p^{k} u
\end{aligned}
$$

Lema 28. Sejam $\alpha_{1}=0$ e $\theta_{1}, \theta_{2}$ as raízes do polinômio principal $Q_{0, F_{1}}(\xi)$ associado a $F_{1}$. Se $\lambda \notin D$ o polinômio $\rho_{\lambda} \rho_{\lambda}^{-1}\left(F_{1}\right)$ resulta preservado e $S o c_{\rho_{\lambda}\left(U_{s}\right)}(N)=W$.

Demonstração. Se $\lambda \in D$, existem inteiros $k_{0}, k_{1}$ tal que $Q_{0, F_{1}}\left(-\lambda+k_{i}\right)=0$, dado que $F_{1}$ é preservado $k_{i}-\lambda \notin \mathbb{Z}^{-}$.

Suponhamos que $M$ um $\rho(\lambda) U s$-submódulo de $\rho(\lambda) U s\left(\begin{array}{l}1 \\ 0\end{array}\right)$ e $n \in \mathbb{N}$ o menor natural tal que $q^{n} u=\left(\begin{array}{c}q^{n} \\ 0\end{array}\right), q^{n} p u=\left(\begin{array}{c}0 \\ q^{n}\end{array}\right) \in M$

Substituindo $k=0$ e $m=n$ em (3.8), segue-se $-q^{n+1} a p u+\left(n(n+\lambda)-b q^{2}\right) q^{n-1} u \in M$. Substituindo $k=1$ em (3.8), temos $q^{n+1} p(-a p u-b u)+(2 m+\lambda+1) q^{n} p^{2} u+n(n+\lambda) q^{n-1} p u \in$ $M$. Dos elementos anteriores segue-se

$$
\begin{aligned}
-f\left(q^{n} p u\right) & =(q p+\lambda+1)\left(-b q^{n} u+(-a q+n) q^{n-1} p u\right) \\
-f\left(q^{n} p^{2} u\right) & =(q p+\lambda+1)\left[\left(q b-q a^{2}+q a^{\prime}+(n+1) a\right) q^{n} p u+\left(q b^{\prime}+(n+1) b-q a b\right) q^{n} u\right] .
\end{aligned}
$$

Se $\lambda \in K$ e $k$ é o maior inteiro tal que $q^{t} p^{k} u \in M$ temos os seguintes elementos de $M$

$$
\begin{aligned}
q^{t+1} p^{k+2} u & =q^{t-1} p^{k}\left(q^{2} p^{2}\right) u-2 k q^{t} p^{k+1} u-k(k-1) q^{t-1} p^{k} u, \\
& \equiv\left(\operatorname{ret}_{0}(b)+k(k-1)\right) q^{t-1} p^{k} u+\left(\operatorname{Res}_{0}(a)+2 k\right) q^{t} p^{k+1} u(\bmod M) . \\
-f\left(q^{t} p^{k} u\right) & =(q p+\lambda+1)\left(q^{t} p^{k+1} u+t q^{t-1} p^{k} u\right) . \\
-f\left(q^{t+1} p^{k+1} u\right) & =(q p+\lambda+1)\left(q^{t+1} p^{k+2} u+(t+1) q^{t} p^{k+1} u\right) .
\end{aligned}
$$

1. Suponhamos que $s_{1}=2, r_{1} \leq 1$. Das equações (3.11) e (3.8) segue $(q p+\lambda+1)\left(Q_{0, F_{1}}(k-\right.$ $\left.n) q^{n} p^{k+1} u\right) \in M$. Como $Q_{0, F_{1}}(k-n) \neq 0$ então $(q p+\lambda+1)\left(q^{n} p^{k+1} u\right), \quad(q p+\lambda+$ 1) $\left(q^{n-1} p^{k} u\right) \in M$. De

$$
\begin{aligned}
& (q p+\lambda+1)\left(q^{n} p^{k+1} u\right)=q^{n+1} p^{k+2} u+(\lambda+n+1) q^{n} p^{k+1} u \\
& (q p+\lambda+1)\left(q^{n-1} p^{k} u\right)=q^{n} p^{k+1} u+(\lambda+n) q^{n-1} p^{k} u
\end{aligned}
$$


segue-se $Q_{0, F_{1}}(-\lambda+k-n) q^{n} p^{k+1} \in M$. Portanto, $q^{n} p^{k+1} \in M$, se e só se, $Q_{0, F_{1}}(-\lambda+$ $k-n) \neq 0$.

2. Suponhamos que $s_{1} \leq 1, r_{1}=1$. Se $\lambda \in \mathbb{Z}^{-}$então $q^{-\lambda} u$ e $q^{-\lambda} p u$ são elementos de $M$. Seja $k$ o maior inteiro positivo tal que $q^{-\lambda} p^{k} u \in M$. De (3.11) temos $\left(\lambda+k+\operatorname{Res}_{0}(a)-1\right) q^{-\lambda} p^{k+1} u \in M$. Se $\lambda+k+\operatorname{Res}_{0}(a)-1=0$ então $\operatorname{Res}_{0}(a)$ é um inteiro menor ou igual a 1 e $k=1-\operatorname{Res}_{0}(a)-\lambda$, $\operatorname{logo} q^{-\lambda} p^{1-\operatorname{Res}(a)-\lambda} u \in M$. Suponhamos que $\lambda \notin \mathbb{Z}^{-}$e sejas $t$ o menor inteiro tal que $Q_{0, F_{1}}(-\lambda-t)=0$ então $q^{t} u, q^{t+1} p u \in M$. Para $k$ o maior inteiro tal que $q^{1+t} p^{k} u \in M$, de (3.8), segue-se $(q p+\lambda+1)\left(t+1-k-\operatorname{Res}_{0}(a)\right) q^{t} p^{k+1} \in M$. Como $-\lambda-t$ é raiz de $Q_{0, F_{1}}(\xi)$ e $\lambda \notin \mathbb{Z}^{-}$ temos $(q p+\lambda+1)\left(q^{t+1} p^{k+1} u\right) \in M$. Portanto, $\left(-t-\lambda+k+\operatorname{Res}_{0}(a)\right) q^{t+1} p^{k+1} u \in M$ e $k=1$.

3. Suponhamos que $s_{1} \leq r_{1}+1$ e $r_{1} \geq 2$. Se $\lambda \in D^{+}$, então $Q_{0, F_{1}}(-\lambda+k)=0$ e $p^{k} u \in M$. Se $\lambda \in D^{-}$, então $Q_{0, F_{1}}(-\lambda-t)=0$ e $q^{t} u, q^{t+1} p u \in M$.

4. Suponhamos que $s_{1} \leq r_{1}, r_{1} \geq 2$. Se $\lambda \in \mathbb{Z}^{-}$, então $q^{-\lambda} u, q^{-\lambda} p u \in M$. De

$$
p^{n+1}\left(q^{r_{1}+1} p^{2} u\right)=\sum c_{j} q^{\left(r_{1}-j+1\right)} p^{\left(n-r_{1}+2\right)+\left(r_{1}-j+1\right)} \in M,
$$

temos $\left(\operatorname{Res}_{0}(b)+(-\lambda+n) \operatorname{Res}_{0}(a)\right) p^{n+1} u \in M$. Portanto, $Q_{0, F_{1}}(-\lambda+n) p^{n+1} u \in M$.

Mostramos que $M$ é um $U_{S^{-}}$submódulo irredutível e maximal de $q^{t} W$, portanto

$$
M=\operatorname{Soc}_{\rho_{\lambda}\left(U_{s}\right)}(N) \text {. }
$$

Além disso $\operatorname{Soc}_{\rho_{\lambda}\left(U_{s}\right)}(N)=W$ se, e somente se, $\lambda \notin D$.

Observação 7. Se $\lambda \in D^{-}$existe $t \in \mathbb{N}$ tal que $Q_{0, F_{1}}(-\lambda-t)=0$. Se $\lambda \in D^{+}$existe $t_{0} \in \mathbb{N}$ tal que $Q_{0, F_{1}}\left(-\lambda+t_{0}\right)=0$.

1. Se $\lambda \in D^{-} \cap D^{+}$então $q^{t} W$ é um submódulo de $W$ e $S o c_{\rho_{\lambda}\left(U_{s}\right)}(N)$ é um submódulo irredutível de $q^{t} W$.

2. Se $\lambda \in D-D^{+}$então $q^{t} W$ é um submódulo irredutível de $W$ e $q^{t} W=\operatorname{Soc}_{\rho_{\lambda}\left(U_{s}\right)}(N)$.

3. Se $\lambda \in D-D^{-}$, então $q^{t} W=W$, com submódulo irredutível $\operatorname{Soc}_{\rho_{\lambda}\left(U_{s}\right)}(N)$.

O quociente $q^{t} W / S_{o \rho_{\lambda}\left(U_{s}\right)}(N)$ é irredutível.

Afirmação 15. Se $\lambda \in D^{+}$, então $V_{\lambda}=q^{t} W / S_{o c} c_{\rho_{\lambda}\left(U_{s}\right)}(N)\left(W / S o c_{\rho_{\lambda}\left(U_{s}\right)}(N)\right)$ é o módulo de $S l_{2}$-módulo de Verma $V(\sigma)$, onde $\sigma$ toma um dos seguintes valores

1. $-\lambda-1$.

2. $-\lambda-t$ se $-\lambda-t$ é raiz de $Q_{0, F_{1}}(\xi)$ para $r_{1}=s_{1}-1$. 
3. $-\lambda+t$ se $-\lambda+t$ é raiz de $Q_{0, F_{1}}(\xi)$ para $r_{1}=s_{1}-1$.

Demonstração. Para a demonstração, usamos a forma do polinômio inicial e a afirmação anterior. Existem inteiros não negativos $t$ mínimo e $k$ máximo tal que $q^{t} p^{k} u \in M$ e $q^{t} p^{k+1} u \notin$ $M$. Definimos

$$
\begin{array}{r}
v_{0}=q^{t} p^{k+1} u, \\
v_{i}=f^{i}\left(q^{t} p^{k+1} u\right) .
\end{array}
$$

Então $q v_{0}=0$, a ação de $h$ sobre $v_{0}$ é dada como segue, em cada caso.

1. Suponhamos que $r_{1} \leq 1$ e $s_{1} \leq 2$. Usando (3.11) nos elementos $q^{t} p^{k}, q^{t+1} p^{k+1}$ temos

$$
\begin{aligned}
(q p+\lambda+1)\left(q^{t} p^{k+1} u\right) & \equiv q^{t+1} p^{k+2} u+(\lambda+1+t) q^{t} p^{k+1} u & & (\bmod M), \\
q^{t+1} p^{k+2} u & \equiv-(\lambda+1+t) q^{t} p^{k+1} u & & (\bmod M) .
\end{aligned}
$$

Da equação anterior e de $h v_{0}=2 q^{t+1} p^{k+2} u+(\lambda+1+2 t) q^{t} p^{k+1} u$ obtemos

$$
h v_{0} \equiv-(\lambda+1) q^{t} p^{k+1} u
$$

2. Suponhamos que $r_{1} \geq 2$ e $s_{1}=r_{1}+1$. Se $Q_{0, F_{1}}(-\lambda-t)=0$ para $t \in \mathbb{N}$, da Lema anterior $q^{t} u, q^{t+1} p u \in M$. Definimos os seguinte elementos

$$
\begin{aligned}
& v_{0}=q^{t-1} u, \\
& v_{i}=f^{i} v_{0} .
\end{aligned}
$$

Então $q v_{0} \equiv 0(\bmod M)$. De $q p\left(q^{t+1} p u\right)$ temos $w_{0}=r s t_{0}(b) q^{t-2} u+\operatorname{Res}_{0}(a) q^{t-1} p u \in M$. De $w_{0}$ e $f\left(q^{t+1} p u\right)$ geramos o elemento $(q p+\lambda+1)\left(q^{t-1} u\right) \in M$ então $-(\lambda+t) q^{t-1} u \equiv$ $q^{t} p u(\bmod M)$. Portanto,

$$
\begin{aligned}
(2 q p+\lambda+1)\left(q^{t-1} u\right) & =2 q^{t} p u+(\lambda+t) q^{t-1} u \\
& \equiv-(\lambda+t) q^{t-1} u \quad(\bmod M)
\end{aligned}
$$

Se $Q_{0, F_{1}}(-\lambda+t)=0$ para $t \in \mathbb{N}$ a demonstração é similar, basta substituir $q^{t} u$ por $p^{t} u$. No caso $s_{1}<r_{1}$ a demonstração é similar.

Finalmente temos o teorema principal do trabalho, a demonstração do teorema é uma consequência de todo o trabalho feito até agora.

Teorema 27. Seja $N$ um $\mathfrak{B}$-módulo irredutivel de grau dois e $\lambda \in K$. Da identificação $N \equiv K(q) \times K(q)$, onde $p$ e $q$ agem como a multiplicação por esquerda pelas matrizes $\left(\begin{array}{cc}\frac{d}{d q} & -b \\ 1 & \frac{d}{d q}-a\end{array}\right)$ e $\left(\begin{array}{cc}q & 0 \\ 0 & q\end{array}\right)$, respetivamente. Então

$$
\operatorname{Soc}_{\rho(\lambda) U s} N=\operatorname{Soc}_{A_{1}} N
$$


salvo que,

$$
\begin{array}{ccc}
0 \notin \operatorname{Pol}\left(F_{1}\right) & \text { e } & \lambda \notin \mathbb{Z}^{-}, \\
0 \in \operatorname{Pol}\left(F_{1}\right) & e & -\lambda \in D .
\end{array}
$$

Além disso, se $\Delta\left(F_{1}\right)=\emptyset$ temos

$$
A_{1}\left(\begin{array}{l}
1 \\
0
\end{array}\right)=K\left[q,\left(q-\alpha_{1}\right)^{-1}, \cdots,\left(q-\alpha_{n}\right)^{-1}\right] \times K\left[q,\left(q-\alpha_{1}\right)^{-1}, \cdots,\left(q-\alpha_{n}\right)^{-1}\right] .
$$

No outro caso, $A_{1}\left(\begin{array}{l}1 \\ 0\end{array}\right)$ é um submódulo irredutivel de

$$
K\left[q,\left(q-\alpha_{1}\right)^{-1}, \cdots,\left(q-\alpha_{n}\right)^{-1}\right] \times K\left[q,\left(q-\alpha_{1}\right)^{-1}, \cdots,\left(q-\alpha_{n}\right)^{-1}\right] .
$$

Se temos (3.14), então $\rho(\lambda) U s\left(\begin{array}{l}1 \\ 0\end{array}\right)=q^{-\lambda} A_{1}\left(\begin{array}{l}1 \\ 0\end{array}\right)$. Se temos $(3.15)$, então $\operatorname{Soc}_{\rho_{\lambda}\left(U_{s}\right)}(N)=$ $\rho(\lambda) U s\left(\begin{array}{l}1 \\ 0\end{array}\right)$ e existe $t \in \mathbb{N}$ tal que o módulo quociente

$$
q^{t} A_{1}\left(\begin{array}{l}
1 \\
0
\end{array}\right) / \operatorname{Soc}_{\rho_{\lambda}\left(U_{s}\right)}(N)\left(A_{1}\left(\begin{array}{l}
1 \\
0
\end{array}\right) / \operatorname{Soc}_{\rho_{\lambda}\left(U_{s}\right)}(N)\right)
$$

é isomorfo a um dos seguintes módulos de Verma,
1. $M(-\lambda-1)$.
2. $M(-\lambda-t)$, se $Q_{0, F_{1}}(-\lambda-t)=0$ para $t \in \mathbb{N}$.
3. $M(-\lambda+t)$, se $Q_{0, F_{1}}(-\lambda+t)=0$ para $t \in \mathbb{N}$.

No caso $\lambda \in D^{+} \cap D^{-}$o módulo $W / \operatorname{Soc}_{\rho_{\lambda}\left(U_{s}\right)}(N) \simeq V(k)$, onde $k \in \mathbb{N}$ satisfazendo $\lambda=k+\theta_{i}$. 


\section{Capítulo 4}

\section{Extensões De Módulos irredutíveis}

Nesta sessão, estudaremos as extensões de $A_{1}$-módulos irredutíveis. Sejam $M, N$ dois $A_{1^{-}}$ módulos irredutíveis. Para $V \in \operatorname{Ext}_{A_{1}}^{1}(N, M)$ temos a seguinte sequência exata de $A_{1^{-}}$ módulos

$$
0 \longrightarrow M \stackrel{\varphi}{\longrightarrow} V \stackrel{\psi}{\longrightarrow} N \longrightarrow 0
$$

Tomando a localização por $S=K[q]-\{0\}$ obtemos a sequencia exata de $\mathfrak{B}$-módulos

$$
0 \longrightarrow S^{-1} M=\mathfrak{B} /\left(\mathfrak{B} G_{1}\right) \stackrel{\hat{\varphi}}{\longrightarrow} S^{-1} V \stackrel{\hat{\psi}}{\longrightarrow} S^{-1} N=\mathfrak{B} /\left(\mathfrak{B} G_{2}\right) \longrightarrow 0,
$$

então $S^{-1} V \simeq \mathfrak{B} /\left(\mathfrak{B} G_{1} G_{2}\right)$.

Observação 8. Seja $F \in \mathfrak{B}$ irredutível e não preservado, então $A_{1} \cap \mathfrak{B} F$, não é um $A_{1^{-}}$ módulo maximal de $A_{1}$, mais ainda $F$ não é livre de $S$-torção.

Suponha $F \in \operatorname{irr}(\mathfrak{B})$ não é preservado, então o $A_{1}$-módulo $W=A_{1} /\left(A_{1} \cap \mathfrak{B} F\right)$, não é irredutível. Seja $f \in K[q]$, de grau mínimo tal que $F f^{-1}$ é preservando, temos os seguintes isomorfismos de $A_{1}$-módulos

$$
\left(A_{1} f+A_{1} \cap \mathfrak{B} F\right) /\left(A_{1} \cap \mathfrak{B} F\right) \simeq A_{1} f /\left(A_{1} f \cap \mathfrak{B} F\right) \simeq A_{1} /\left(A_{1} \cap \mathfrak{B} F f^{-1}\right) .
$$

Logo, o módulo $V_{f}=A_{1} /\left(A_{1} \cap \mathfrak{B} F f^{-1}\right) \leq W$ é um $A_{1}$-submódulo irredutível, e o módulo quociente $W / V_{f}$ é de torção. Por outro lado o módulo $W$ é livre de $S$-torção. Suponha que existem $w \in W$ e $s \in S$ tais que $s w \in A_{1} \cap \mathfrak{B} b$, então $w \in A_{1} \cap \mathfrak{B} b$ e $w=0$.

Para todos os elementos $g \in K[q]$ tal que $F g^{-1}$ é preservado, temos $g=d f$, onde $f \in K[q]$ é o polinômio de menor grau satisfazendo esta condição. Suponha $g \in K[q]$ tal que $F g^{-1}$ é preservado. Dado que $f$ é de grau minimal, existem $g_{1}, g_{2}$ tais que $g=g_{1} f+g_{2}$, onde $\operatorname{grau}\left(g_{2}\right)<\operatorname{grau}(f)$. Notemos que $Q_{\alpha, b g_{2}^{-1}}(\xi)=\lambda Q_{\alpha, b}\left(\xi-v_{\alpha\left(g_{2}\right)}\right)$, e $Q_{\alpha, b}\left(\xi-v_{\alpha}(f)\right)$, não tem raízes inteiras negativas, então $Q_{\alpha, b g_{2}^{-1}}(\xi)$ não tem raízes inteira negativas, portanto $g_{2}=0$.

Exemplo 7. Suponhamos que $N$ é irredutível de $S$-torção, e $M$ é irredutível de grau $n$ em (4.1). Seja $v \in V$ tal que $\psi(v)=n \in N-\{0\}$ e $(q-\alpha) n=0$ então $(q-\alpha) v \in \operatorname{Im}(\varphi)$. Para todo $w \in V$ existe $f \in A_{1}$ tal que $w=f v+\psi(m)$. Se $w \in \operatorname{Im}(\varphi) \cap A_{1} v$ e $(q-\alpha) v=0$, então $w=0$. Portanto, $V \simeq M \oplus N$. Se $(q-\alpha) v \neq 0$ existe $m \in M$ tal que $\varphi(m)=(q-\alpha) v$, assim $V=A_{1} v$. Como $M$ é irredutivel e livre de $S$-torção, existe $F \in \mathfrak{B}$ de grau minimal irredutível, 
preservado e anulador de $m$ que satisfaz $F(q-\alpha) v=0$ e $V \simeq A_{1} /\left(A_{1} \cap \mathfrak{B} F(q-\alpha)\right)$ com sub-módulo irredutível isomorfo a $A_{1} /\left(A_{1} \cap \mathfrak{B} F\right) \simeq M$.

Do exemplo anterior é natural se perguntar pela relação que existe entre os módulos $A_{1} F$ e $A_{1} \cap \mathfrak{B} F$. Daremos a relação que existe entre os ideais $A_{1} F$ e $A_{1} \cap \mathfrak{B} F$ na seguinte proposição.

Proposição 2. Suponhamos que $N$ irredutivel de grau $n$ tal que $N \simeq A_{1} /\left(A_{1} \cap \mathfrak{B} F\right)$, onde $F=\sum b_{i} p^{i} \in A_{1}$ é irredutivel e preservado de grau minimo na variável $p$, e os $b_{i}$ são relativamente primos. Então $\left(A_{1} \cap \mathfrak{B} F\right) / A_{1} F$ é de $S$-torção. Basta provar que se $G \in$ $A_{1} \cap \mathfrak{B} F$, então $b_{n}^{g r_{p}(G)} G \in A_{1} F$.

Demonstração. Seja $G \in A_{1} \cap \mathfrak{B} F$, a demonstração é feita por indução no grau de $G$ em $p$. Se $\operatorname{deg}_{p}(G)=g r_{p}(F)$, então $G \in A_{1} F$. Suponha que para todo polinômio de grau em $p$ menor o igual que $k+n$, a afirmação e valida. Seja $G \in A_{1} \cap \mathfrak{B} F$ tal que $g r_{p}(G)=k+n+1$ e $f_{k+n+1} \neq 0$, o coeficiente principal de $G$. Então o grau em $p$ de $G_{1}=b_{n} G-f_{k+n+1} p^{1+k} F$ é $k+n$, e $b_{n}^{k+n} G_{1} \in A_{1} F$. Logo,

$$
b_{n}^{k+n+1} G \in A_{1} F .
$$

Das afirmações anteriores temos

$$
\left(A_{1} \cap \mathfrak{B} F\right) / A_{1} F \simeq \sum_{\alpha \in \Delta(F)} \bigoplus M(\alpha)
$$

Onde $M(\alpha)$ é um $A_{1}$-módulo irredutível de $S$-torção de peso $\alpha$.

Afirmação 16. Usando a notação anterior, suponhamos que $M$ é um $A_{1}$-módulo irredutível de $S$-torção e $N$ irredutivel de grau $n$, então $V \simeq M \oplus N$ ou $V \simeq A_{1} / A_{1} F$. Além disso $M \simeq A_{1} F /\left(A_{1}(q-\lambda)^{t} f\right)$ para $f \in\left(A_{1} \cap \mathfrak{B} F\right)-J$ e $t \in \mathbb{N}$ suficientemente grande.

Demonstração. Seja $v \in V$ tal que $\psi(v)=\overline{1}$, então $F v=\varphi(m)$. Além disso, para cada $x \in V$ existem $f \in A_{1}$ e $m \in M$ tais que $x=f v_{\varphi}(m)$. Definimos $J$ como o ideal a esquerda de $A_{1}$ que contém todos os elementos $f \in A_{1} \cap \mathfrak{B} F$ com a propriedade; $f v=0$.

1. Suponhamos que $\alpha \notin \operatorname{Pol}\left(F_{1}\right)$. Para cada $f \in A_{1} \cap \mathfrak{B} F$ e $l, t \in \mathbb{N}$ temos $(q-\alpha)^{t} f v+$ $b_{n}^{l} f v=f v$. Se $F v=0$ obtemos $J=A_{1} \cap \mathfrak{B} b$ e $V=M \bigoplus N$.

Se $F v \neq 0$ então $A_{1} F+J=A_{1} \cap \mathfrak{B} F$ e $V=A_{1} / J$, e ainda $M=A_{1} F /\left(A_{1}(q-\alpha)^{t} F\right)$ onde $t$ é o menor inteiro tal que $(q-\alpha)^{t} F v=0$.

2. Suponhamos que $\alpha \in \operatorname{Pol}\left(F_{1}\right)$. Para $J=A_{1} \cap \mathfrak{B} F$ temos $V=M \bigoplus N$. Se $J \neq A_{1} \cap \mathfrak{B} F$ escolhemos $f \in A_{1} \cap \mathfrak{B} F$ de grau mínimo tal que $f v \neq 0$. Portanto, $J+A f=A_{1} \cap \mathfrak{B} F$, $V=A_{1} / J$ e $M=A_{1} f /\left(A_{1}(q-\alpha)^{t} f\right)$, onde $t$ é o menor inteiro tal que $(q-\alpha)^{t} f v=0$.

Suponhamos que $F_{1}, F_{2} \in \mathfrak{B}$ são irredutíveis, se $V \in \operatorname{Ext}^{1}\left(\mathfrak{B} / \mathfrak{B} F_{1}, \mathfrak{B} / \mathfrak{B} F_{2}\right)$ e $V \neq$ $\mathfrak{B} / \mathfrak{B} F_{1} \oplus \mathfrak{B} / \mathfrak{B} F_{2}$ então $V$ é um $A_{1}$-módulo cíclico.

Seja $V$ um $A_{1}$-módulo cíclico como acima. Suponhamos que $W=\mathfrak{B} /\left(\mathfrak{B} F_{1} F_{2}\right) \simeq \mathfrak{B} /(\mathfrak{B} G)$, então $F_{1} F_{2} \simeq G$. Assim temos $G=G_{1} \cdots G_{r} \operatorname{com} G_{j} \in \operatorname{irr}(\mathfrak{B})$ e $g r(G)=\operatorname{gr}\left(G_{1} \cdots G_{r}\right)$. Por 
outro lado o $\mathfrak{B}$-módulo $W=\mathfrak{B} /\left(\mathfrak{B} F_{1} F_{2}\right)$ tem um único sub-módulo $W_{2}=\mathfrak{B} F_{2} /\left(\mathfrak{B} F_{1} F_{2}\right)$, então $r=2$, e consequentemente temos o isomorfismo entre os quocientes

$$
W / W_{2} \simeq \mathfrak{B} b_{2} /\left(\mathfrak{B} G_{1} G_{2}\right)
$$

concluímos que $F_{2} \simeq G_{2}$ e pelo argumento no artigo de Oystein Ore (Ore , 1933)pag.494 Teorema1, se segue que $F_{1} \simeq G_{1}$.

Sejam $M_{1}, M_{2} \quad A_{1}$-módulos cíclicos. Suponhamos que $S^{-1} M_{1} \simeq S^{-1} M_{2}$ então $S^{-1} M_{i} \simeq$ $\mathfrak{B} /\left(\mathfrak{B} F_{i 1} F_{i 2}\right)$. Deduzimos do anterior; $A_{1} /\left(A_{1} \cap \mathfrak{B} F_{11} F_{12}\right) \simeq A_{1} /\left(A_{1} \cap \mathfrak{B} F_{21} F_{22}\right)$. Isto mostra que a aplicação $S^{-1}$ é injetiva. Ver que $S^{-1}$ é sobrejetora, é imediato.

Afirmação 17. Suponha $M$ e $N A_{1}$-módulos irredutiveis de grau $t_{1}, t_{2}$ respectivamente, então o $\operatorname{Ext}_{A_{1}}^{1}(M, N)$ contem duas classes de módulos com representantes $M \oplus N e V \simeq$ $A_{1} /\left(A_{1} \cap \mathfrak{B} F_{1} F_{2}\right)$, onde $F_{1}$ é o anulador de $m \in M-\{0\}$ e $F_{2}$ é o anulador de algum $n \in N-\{0\}$.

Demonstração. Da (4.1) temos $V=A_{1} v$ para algum $\psi(v) \in N-\{0\}$ ou $V \simeq M \oplus N$. Além disso $F_{2} v \in \operatorname{Im}(\varphi)$ e $F_{1} F_{2} v=0 \operatorname{com} F_{1}, F_{2} \in A_{1}$. Se $F_{2} v \neq 0$ então $V \simeq A_{1} /\left(A_{1} \cap \mathfrak{B} F_{1} F_{2}\right)$. Se $F_{2} v=0$, então $V \simeq M \oplus N$.

\subsection{Análise do caso $F \in A_{1} \operatorname{com} F$ redutível em $\mathfrak{B}$}

Suponhamos que $F \in A_{1}$ onde $F=F_{1} F_{2} \operatorname{com} F_{i} \in \operatorname{irr}(\mathfrak{B})$ e $\operatorname{deg}_{p}\left(b_{i}\right)>1$. Vamos construir a cadeia de comprimento de $A_{1}$-módulos,

$$
\left(A_{1} f_{F_{1}} F_{2}+A_{1} F_{1} F_{2}\right) / A_{1} F_{1} F_{2} \leq A_{1} F_{2} / A_{1} F_{1} F_{2} \leq\left(A_{1} f_{F_{2}}+A_{1} F_{2}\right) / A_{1} F_{1} F_{2} \leq A_{1} / A_{1} F_{1} F_{2} .
$$

Sejam $f_{F_{i}} \in K[q]$ de grau mínimo tal que $F_{i} f_{F_{i}}^{-1}$ é preservado. Temos os seguintes isomorfismos

1. $\left(A_{1} / A_{1} F_{1} F_{2}\right) /\left[\left(A_{1} f_{F_{2}}+A_{1} F_{2}\right) / A_{1} F_{1} F_{2}\right] \simeq A_{1} /\left(A_{1} f_{F_{2}}+A_{1} F_{2}\right)$.

2. $A_{1} f_{F_{2}} /\left(A_{1} f_{F_{2}} \cap A_{1} F_{2}\right) \simeq A_{1} /\left(A_{1} \cap \mathfrak{B} F_{2} f_{F_{2}}^{-1}\right)$.

3. $A_{1} F_{2} / A_{1} F_{1} F_{2} \simeq A_{1} / A_{1} F_{1}$.

4. $\left(A_{1} F_{2} / A_{1} F_{1} F_{2}\right) /\left[\left(A_{1} f_{F_{1}} F_{2}+A_{1} F_{1} F_{2}\right) / A_{1} F_{1} F_{2}\right] \simeq A_{1} /\left(A_{1} f_{F_{1}}+A_{1} F_{1}\right)$.

Considere o $A_{1}$-módulo $V_{12}=A_{1} / A_{1} F_{1} F_{2}$. Como $A_{1} F_{1} F_{2} \subseteq A_{1} F_{2}$, podemos definir o $A_{1^{-}}$ submódulo $V_{2}=A_{1} F_{2} / A_{1} F_{1} F_{2} \subset V_{12}$, por outro lado temos isomorfismo $V_{2} \simeq A_{1} / A_{1} F_{1}$ induzido pelo homomorfismo

$$
\varphi: A_{1} \longrightarrow V_{2}
$$

que é dado por $\varphi(a)=a F_{2} \operatorname{com} \operatorname{ker}(\varphi)=A_{1} F_{1}$. Ver (Ore, 1933).

Temos a sequência de sub-módulos $V_{12} \geq V_{b_{2}}=\left(A_{1} f_{F_{2}}+A_{1} F_{2}\right) / A_{1} F_{1} F_{2} \geq V_{2}$, com a seguintes propriedades:

1. $V_{F_{2}} / V_{2} \simeq A_{1} /\left(A_{1} \cap \mathfrak{B} F_{2} f_{F_{2}}^{-1}\right)$. Seguem os seguinte isomorfismos de $A_{1}$-módulos

$$
V_{F_{2}} / V_{2} \simeq\left(A_{1} f_{F_{2}}+A_{1} F_{2}\right) / A_{1} F_{2} \simeq A_{1} f_{F_{2}} /\left(A_{1} f_{F_{2}} \cap A_{1} F_{2}\right) \text {. }
$$


Considere o homomorfismo $\varphi_{2}: A_{1} \longrightarrow A_{1} f_{F_{2}} /\left(A_{1} f_{F_{2}} \cap A_{1} F_{2}\right)$ dado por $\varphi_{2}(a)=$ $a f_{F_{2}}+\left(A_{1} f_{F_{2}} \cap A_{1} F_{2}\right)$ com $\operatorname{ker}\left(\varphi_{2}\right)=A_{1} \cap \mathfrak{B} F f_{F_{2}}^{-1}$. Logo, $V_{F_{2}} / F_{2}$ é irredutível.

2. Do item anterior obtemos $\operatorname{Soc}_{A_{1}}\left(V_{2}\right) \leq V_{2} \leq V_{F_{2}} \leq V_{12}$. Do homomorfismo $\varphi$ temos $\varphi\left(\left(A_{1} f_{F_{1}}+A_{1} F_{1}\right) / A_{1} F_{1}\right) \simeq\left(A_{1} f_{F_{1}} F_{2}+A_{1} F_{1} F_{2}\right) / A_{1} F_{1} F_{2}$. Pelo item anterior

$$
\left(A_{1} f_{F_{1}} F_{2}+A_{1} F_{1} F_{2}\right) / A_{1} F_{1} F_{2}
$$

é irredutível, assim $\operatorname{Soc}_{A_{1}}\left(V_{2}\right)=\left(A_{1} f_{F_{1}} F_{2}+A_{1} F_{1} F_{2}\right) / A_{1} F_{1} F_{2}$.

Agora estudaramos os quocientes $V_{12} / V_{F_{2}}$ e $V_{2} / \operatorname{Soc}_{A_{1}}\left(V_{2}\right)$, sabendo que estes são módulos de $S$-torção.

Seja $F \in \operatorname{irr}(\mathfrak{B})$ tal que $F=\sum a_{j} p^{j}$ e $f_{F}$, como foi definido anteriormente. Então

$$
A_{1} / A_{1} F \geq\left(A_{1} f_{F}+A_{1} F\right) / A_{1} F \simeq A_{1} f_{F} /\left(A_{1} f_{F} \cap A_{1} F\right) \simeq A_{1} /\left(A_{1} \cap \mathfrak{B} F f_{F}^{-1}\right),
$$

com $A_{1} /\left(A_{1} f_{F}+A_{1} F\right)$ de $S$-torção. Como $f_{F}=\prod\left(q-\alpha_{i}\right)^{\theta_{\alpha_{i}}(F)}$, temos

$$
\begin{aligned}
A_{1} /\left(A_{1} f_{F}+A_{1} F\right) & =A_{1} / A_{1} F \bigotimes_{K[q]} K[q] /\left(K[q] f_{F}\right) \\
& \simeq \bigoplus A_{1} F \bigotimes_{K[q]} K[q] /\left(K[q]\left(q-\alpha_{i}\right)^{\theta_{\alpha_{i}}(F)}\right) .
\end{aligned}
$$

Para o primeiro isomorfismo, definimos

$$
\phi: A_{1} / A_{1} F \times K[q] /\left(K[q] f_{F}\right) \longrightarrow A_{1} /\left(A_{1} f_{F}+A_{1} F\right),
$$

dado por $\phi\left(a+A_{1} F, c+K[q] f\right)=a c+\left(A_{1} f_{F}+A_{1} F\right)$, note que $\phi$ está bem definido e $K[q]$-bilinear. Pela propriedade universal do produto tensorial, existe um homomorfismo de $A_{1}$-módulos

$$
\hat{\phi}: A_{1} / A_{1} F \bigotimes_{K[q]} K[q] /\left(K[q] f_{F}\right) \longrightarrow A_{1} /\left(A_{1} F_{f}+A_{1} F\right)
$$

portanto $\hat{\phi}$ é um isomorfismo. 


\section{Referências Bibliográficas}

Amitsur (1954) A. S. Amitsur. Differential polynomials and division algebras. Ann. of Math. (2), 59:245-278. ISSN 0003-486X. Citado na pág. viii

Bavula (2005) V.V. Bavula. Dixmier's problem 5 for the weyl algebra. Journal of Algebra, 283(2):604 - 621. Citado na pág. viii

Block (1981) R. E. Block. The irreducible representations of the lie algebras $\operatorname{sl}(2)$ and the weyl algebras. Adv. Math, 39:69-110. Citado na pág. iii, v, viii, ix, 8, 12, 13, 14, 15, 16, 17, 19,23

Block (1979) Richard E. Block. The irreducible representations of the Weyl algebra $A_{1}$. Em Séminaire d'Algèbre Paul Dubreil 31ème année (Paris, 1977-1978), volume 740 of Lecture Notes in Math., páginas 69-79. Springer, Berlin. Citado na pág. viii

Cartan (1894) E. Cartan. Sur la structure des groupes de transformations finis et continus. thesis, Nony, Paris, and Oeuvres Completes, Tome 1:137-287. Citado na pág. vii

Coutinho (1995) S. C. Coutinho. A primer of algebraic D-modules. London Mathematical Society student texts 33. Cambridge University Press. ISBN 0521551196,9780521551199,0521559081. Citado na pág.

Dixmier (1963) J. Dixmier. Représentations irréductibles des algèbres de lie nilpotentes. An. Acad. Brasil. Ci., 35:491-519. ISSN 0001-3765. Citado na pág. vii, 1, 3, 4, 5, 6, 7

Dixmier (1977a) J. Dixmier. Idéaux primitifs dans les algèbres enveloppantes. J. Algebra, 48(1):96-112. ISSN 0021-8693. Citado na pág.

Dixmier (1959) Jacques Dixmier. Sur l'algèbre enveloppante d'une algèbre de lie nilpotente. Arch. Math., 10:321-326. ISSN 0003-9268. Citado na pág. iii, vii

Dixmier (1968) Jacques Dixmier. Sur les algèbres de Weyl. Bull. Soc. Math. France, 96: 209-242. ISSN 0037-9484. Citado na pág. viii

Dixmier (1977b) Jacques Dixmier. Enveloping algebras. North-Holland Publishing Co., Amsterdam-New York-Oxford. ISBN 0-7204-0430-4. North-Holland Mathematical Library, Vol. 14, Translated from the French. Citado na pág. 1, 5

Humphreys (1972) James E. Humphreys. Introduction to Lie algebras and representation theory. Springer-Verlag, New York-Berlin. Graduate Texts in Mathematics, Vol. 9. Citado na pág. 1

Jacobson (1943) Nathan Jacobson. The Theory of Rings. American Mathematical Society Mathematical Surveys, vol. II. American Mathematical Society, New York. Citado na pág. 
Jacobson (1979) Nathan Jacobson. Lie algebras. Dover Publications, Inc., New York. ISBN 0-486-63832-4. Republication of the 1962 original. Citado na pág. 1

Jacques (1973) Dixmier Jacques. Quotients simples de l'algèbre enveloppante de $s l_{2}(k)$. J. Algebra, 24:551-564. ISSN 0021-8693. Citado na pág. 12

Joseph (1975) A. Joseph. The weyl algebras-semisimples and nipotent elements, no. 3. Amer. J. Math., 97:597-615. Citado na pág. viii

Joseph (1984) A. Joseph. Primitive ideals in enveloping algebras. Em Proceedings of the International Congress of Mathematicians, Vol. 1, 2 (Warsaw, 1983), páginas 403-414. PWN, Warsaw. Citado na pág.

Kovacic (1972) J. Kovacic. An eisenstein criterion for noncommutative polynomials. Poceedings, Amm. Math. Soc., 34, No. 1:25-29. Citado na pág. 25

Levi (1905) E.E. Levi. Sulla strutura dei gruppi finite e continui. Atti. Accad. Sci. Toronto, 40:551-565. Citado na pág. vii

Maltsev (1962) A. I. Maltsev. On solvable lie algebras. Amer. math. Soc. trlations 1950, 9:228-262. Citado na pág. vii

Mazorchuk (2009) Volodymyr Mazorchuk. Lectures on $\mathrm{Sl}_{2}(\mathbb{C})$-modules. World Scientific Book. Citado na pág. 12

McConnell e Robson (1987) J. C. McConnell e J. C. Robson. Noncommutative Noetherian rings. Pure and Applied Mathematics (New York). John Wiley \& Sons, Ltd., Chichester. ISBN 0-471-91550-5. With the cooperation of L. W. Small, A Wiley-Interscience Publication. Citado na pág. viiii, 4

Nouazé (1967) Yvon Nouazé. Remarques sur: "Idéaux premiers de l'algèbre enveloppante d'une algèbre de Lie nilpotente". Bull. Sci. Math. (2), 91:117-124. ISSN 0007-4497. Citado na pág.

Ore (1933) Oystein Ore. Theory of non-commutative polynomials. Ann. of Math. (2), 34 (3):480-508. ISSN 0003-486X. Citado na pág. viii, 14, 17, 49 